HEP-TH/0412331

AEI-2004-098

ULB-TH-05-03

\title{
Planar plane-wave matrix theory at the four loop order: Integrability without BMN scaling
}

\author{
Thomas Fischbacher ${ }^{(a, b)},{\text { Thomas } \operatorname{Klose}^{(a)} \text { And Jan PlefkA }}^{(a)}$ \\ a) Max-Planck-Institut für Gravitationsphysik \\ Albert-Einstein-Institut \\ Am Mühlenberg 1, D-14476 Potsdam, Germany \\ b) Physique Théorique et Mathématique and \\ International Solvay Institutes, Université Libre de Bruxelles, \\ Campus Plaine C.P. 231, B-1050 Bruxelles, Belgium \\ tf, thklose,plefka@aei.mpg.de
}

\begin{abstract}
We study $\mathrm{SU}(N)$ plane-wave matrix theory up to fourth perturbative order in its large $N$ planar limit. The effective Hamiltonian in the closed $\mathfrak{s u}(2)$ subsector of the model is explicitly computed through a specially tailored computer program to perform large scale distributed symbolic algebra and generation of planar graphs. The number of graphs here was in the deep billions.

The outcome of our computation establishes the four-loop integrability of the planar plane-wave matrix model. To elucidate the integrable structure we apply the recent technology of the perturbative asymptotic Bethe ansatz to our model. The resulting S-matrix turns out to be structurally similar but nevertheless distinct to the so far considered long-range spin-chain S-matrices of Inozemtsev, Beisert-Dippel-Staudacher and Arutyunov-Frolov-Staudacher in the AdS/CFT context.

In particular our result displays a breakdown of BMN scaling at the four-loop order. That is, while there exists an appropriate identification of the matrix theory mass parameter with the coupling constant of the $\mathcal{N}=4$ superconformal Yang-Mills theory which yields an eighth order lattice derivative for well separated impurities (naively implying BMN scaling) the detailed impurity contact interactions ruin this scaling property at the four-loop order.

Moreover we study the issue of "wrapping" interactions, which show up for the first time at this loop-order through a Konishi descendant length four operator.
\end{abstract}




\section{Contents}

\begin{tabular}{lll}
\hline & Introduction & 4
\end{tabular}

2 Effective Hamiltonian of plane-wave matrix theorv 6

2.1 The model . . . . . . . . . . . . . . . . . . 6

2.2 Perturbation theorv to fourth order . . . . . . . . . . . . . . . 8

2.3 Hermitization . . . . . . . . . . . . . . . . . . . . . . . . . . . . . . . . . . .

2.4 Short states - wrapping issues . . . . . . . . . . . . . . . . . 13

3 Properties of the PWMT-dilatation operator 14

3.1 Renormalization and naive BMN scaling . . . . . . . . . . . . . . . . 14

3.2 Distinction to Inozemtsev and Beisert-Dippel-Staudacher spin-chains . . . . 17

3.3 Degenerate spectrum and higher charges . . . . . . . . . . . . . . . . 18

4 Bethe ansatz for plane-wave matrix theory 20

4.1 Spin-chain language . . . . . . . . . . . . . . . . . . 20

4.2 Two-magnon scattering $\ldots \ldots \ldots \ldots \ldots$

4.3 Bethe equations. . . . . . . . . . . . . . . . . . . . . . 24

4.4 Two-magnon spectrum and violation of BMN scaling . . . . . . . . . . . 25

\begin{tabular}{|lr}
5 Conclusion and discussion & 26
\end{tabular}

A Details on the Computation 29

A.1 The Task . . . . . . . . . . . . . . . . . . . . . . 29

A.2 Choice of the Platform . . . . . . . . . . . . . . . . . . . 32

A.3 Planar Graph Generation . . . . . . . . . . . . . . . . . . 32

A.4 Operator Sequences . . . . . . . . . . . . . . . . . . 34

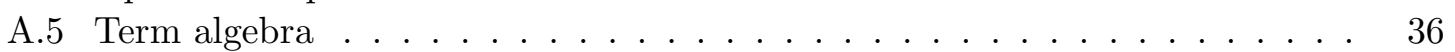

A.5.1 Rules . . . . . . . . . . . . . . . . . 36

A.5.2 Strategr . . . . . . . . . . . . . . . . . . 37

A.5.3 On the Graph $\leftrightarrow$ Term interplav . . . . . . . . . . . . . . . . . . 38

A.5.4 Implementation . . . . . . . . . . . . . . . . . . . . . 41

A.6 On the Calculation . . . . . . . . . . . . . . . . . . . . . . 41

A.6.1 Performance . . . . . . . . . . . . . . . . . 42

A.6.2 Distributed Computing . . . . . . . . . . . . . . . . 43

A.6.3 Securitv . . . . . . . . . . . . . . . . . . 44

A.6.4 On the Code . . . . . . . . . . . . . . . . . . 45

A.7 Five Loops . . . . . . . . . . . . . . . . . . . . . . . . 45

A.8 Results. . . . . . . . . . . . . . . . . . . . . . . . . . . . . . . 46 
B Perturbation expansion of effective Hamiltonian 48

\begin{tabular}{|l|l|}
\hline C Higher charges & 51
\end{tabular}

$\begin{array}{ll}\text { D Spin chain spectrum } & 53\end{array}$ 


\section{Introduction}

In this work we report on a high order perturbative study of plane-wave matrix theory [1] in its large $N$ limit, which is a supersymmetric, mass deformed $\mathrm{SU}(N)$ gauge quantum mechanical model intricately related to maximally supersymmetric four dimensional YangMills theory through a consistent reduction on $\mathbb{R} \times \mathbb{S}^{3}[2]$. It contains quite involved technical parts, in particular on the side of realizing this computation through a specially tailored computer program designed by one of the authors (TF) to perform large scale distributed symbolic algebra for the computation of planar diagrams. Readers interested mostly in the computer science aspects of this computation are advised to directly proceed to App. A which is largely self contained.

The motivation for performing this computation grew out of the mounting evidence for integrability of planar $\mathcal{N}=4$ super Yang-Mills theory (SYM), which should be of prime importance in the AdS/CFT correspondence. In a nutshell it states that the dilatation operator of the planar gauge theory, which yields the anomalous dimensions of local composite operators in the conformal quantum field theory, is given by a long-range integrable spin chain Hamiltonian [3, 4, 5, 6, 7, [8] ${ }^{1}$. This statement is by now firm at the one-loop level for the full theory in form of a (non-compact) $\mathfrak{s u}(2,2 \mid 4)$ super spin-chain [5] and has been extended to the three-loop level in a closed supersymmetric $\mathfrak{s u}(2 \mid 3)$ subsector [6]. In this picture the loop order of the considered dilatation operator is linked to the spread of the local spin interactions of the spin-chain Hamiltonian. In the smallest compact closed subsector of the $\mathcal{N}=4$ gauge theory, the $\mathfrak{s u}(2)$ sector, the l-loop order contribution corresponds to a $s=1 / 2$ spin-chain Hamiltonian of Heisenberg type involving the interactions of $l+1$ neighboring spins [4]. Next to integrability a key property of the established form of the dilatation operator is its perturbative "BMN scaling", which is also predicted from the dual plane-wave superstring [1. This scaling property concerns the form of the $l$ th loop contribution to the energy eigenvalue (or anomalous scaling dimension) in the thermodynamic limit of large spin-chains: its leading contribution scales as $1 / L^{2 l}$, where $L$ is the spin-chain length.

In [7] one of the few known long range integrable spin-chain models of statistical mechanics due to Inozemtsev [10] was identified as being able to provide the established form of the super Yang-Mills dilatation operator up to the three-loop level. This is of course very exciting, as this model could thus provides us with exact non-perturbative information on the $\mathcal{N}=4$ gauge theory. However, as was explicitly demonstrated in [7, the Inozemtsev chain predicts a breakdown of the BMN scaling property at four-loop order. We wish to stress that such a breakdown of perturbative BMN scaling is not necessarily at clash with the AdS/CFT correspondence, despite the BMN scaling property of the dual planewave superstring spectrum. A similar breakdown is by now firmly established in the "near" plane-wave superstring [11] and in the matching of energies of rotating solitonic $A d S_{5} \times S^{5}$ string solutions [12] at three-loop order in perturbative gauge theory. This discrepancy has been attributed to an order of limits problem, stating that a perturbative agreement should not be expected [8]. Hence, based on these results there presently is no reason to expect

\footnotetext{
${ }^{1}$ For a recent review see 9 .
} 
perturbative BMN scaling in $\mathcal{N}=4$ super Yang-Mills! ${ }^{2}$

Nevertheless the assumption of perturbative BMN scaling is a very fruitful one. Indeed Beisert, Dippel and Staudacher [8] have argued that the $\mathfrak{s u}(2)$ dilatation operator is completely determined up to the five loop level once one assumes integrability, BMN scaling and structural constraints from the underlying Feynman diagrammatic structure. These authors went on to conjecture an intriguingly simple all loop (asymptotic) Bethe ansatz, capable of reproducing the eigenvalues of the explicitly deduced dilatation operator up to five loops. Inspired by this structure a similar but distinct asymptotic Bethe ansatz for the full quantum $A d S_{5} \times S^{5}$ string in the $\mathfrak{s u}(2)$ subsector was conjectured by Arutyunov, Frolov and Staudacher in [14. This ansatz accounts for the above mentioned three-loop discrepancies and remarkably reproduces all presently known data of the string side of the correspondence: In its thermodynamic limit it reduces to the Bethe equations of the classical spinning string sigma model of [15], it reproduces the near plane-wave corrections to the quantum string spectrum deduced in [1] and leads to the expected $\lambda^{1 / 4}$ behaviour of the spectrum at strong coupling. The associated spin-chain Hamiltonian to the first few orders in a small $\lambda$ expansion was established in [16].

Given these results it would clearly be of central importance to determine the exact form of the $\mathfrak{s u}(2)$ dilatation operator at the four-loop level and determine whether it obeys BMN scaling and is indeed integrable. This, however, appears to be beyond present capability in the perturbative quantum field theory, where the state of the art is at three-loop order [17, 18, 19.

Our work adds a further piece to this puzzle by considering a reduced (toy) model of the full quantum conformal field theory. The scaling dimensions of the gauge theory on $\mathbb{R}^{4}$ are equivalent to the spectrum of energies in a radial quantization of the model on $\mathbb{R} \times \mathbb{S}^{3}$. It turns out that one may consistently truncate the Kaluza-Klein spectrum of states of $\mathcal{N}=4 \mathrm{SYM}$ on the three-sphere to obtain a reduced gauge quantum mechanical model 2]. The resulting reduced model is the plane-wave matrix theory (PWMT), which has also been proposed as a microscopic definition of M-theory in a maximally supersymmetric eleven-dimensional plane-wave background ${ }^{3}$. Moreover, as was shown in [21], it not only inherits the planar integrability of the $\mathcal{N}=4$ gauge theory, but leads to precisely the same dilatation operator in the closed $\mathfrak{s u}(2)$ subsector up to the three loop level, where the SYM information is firm. In order to perform this matching one has to establish a relation between the coupling constants of the full and the reduced model, which is corrected order by order in perturbation theory. In fact, this relation is uniquely determined by the requirement of BMN scaling behavior.

In this paper we push the perturbation theory of PWMT to fourth order. We explicitly compute the planar effective Hamiltonian in the $\mathfrak{s u}(2)$ subsector without relying on any

\footnotetext{
${ }^{2}$ In the literature arguments for the mechanism of an all loop BMN scaling property of $\mathcal{N}=4 \mathrm{SYM}$ have been put forward in [13. In particular our finding of the "naive" BMN scaling property is in line with these arguments, the breakdown of BMN scaling arises from subtle boundary effects when two impurities come close to each other to be discussed in section 4 .

${ }^{3}$ It also arises as a regularization of the light-cone supermembrane in this background, or alternatively from the dynamics of D0-branes in type IIA string theory [20]
} 
assumptions. The model remains integrable at this loop order but ceases to obey BMN scaling: the leading behavior of the energy eigenvalues scales as $1 / L^{7}$ - after one performs the above mentioned renormalization of coupling constant. Indeed the obtained four-loop contribution is neither of the Inozemtsev nor of the Beisert, Dippel, Staudacher type, while it clearly agrees with these spin-chains up to the three-loop level.

We elucidate the underlying integrable structure of the system by constructing the associated "perturbative asymptotic Bethe ansatz", a novel concept very recently developed in [22]. The extracted S-matrix turns out to qualitatively agree with the quantum string S-matrix of Arutyunov, Frolov and Staudacher [14] but quantitatively disagrees by an additional power of the coupling constant in the exponential term involving the higher charges - which is responsible for the breakdown of BMN scaling. This finding lends support to the suspicion that the generic form of a long-range integrable spin chain S-matrix will involve an extra exponential factor with a characteristic dependence on the higher charges of the spin-chain, first proposed in [14] and further developed in 22].

Furthermore we can shed light on the structure of the "wrapping" interactions, which are inaccessible in the asymptotic forms of the presently conjectured all loop Bethe ansätze. At four-loop order (including five neighboring spin interactions) we have for the first time a non-protected operator, which is shorter than the spread of the dilatation operator: a Konishi descendant of length four. It turns out that no natural prescription of how to act with the five spin terms on this shorter state (wrapping or dropping) reproduces the explicitly computed scaling dimension.

On the computational side, the four-loop study was achieved with a newly developed computer program which constructs all planar diagrams of the perturbation expansion and performs the corresponding algebra. A change of the platform and the use of heavily improved algorithms led to a performance increase by a factor of more than 100 with respect to the previous version when comparing the three-loop computation times. Only this enhancement brought fourth order to reach, but it still required some $88.000 \mathrm{CPU}$ hours on current computers.

\section{Effective Hamiltonian of plane-wave matrix theory}

\subsection{The model}

The Hamiltonian of plane-wave matrix theory can be written as $H=H_{0}+V_{1}+V_{2}$ with

$$
\begin{aligned}
H_{0} & =\operatorname{tr}\left[\frac{1}{2} P_{I} P_{I}+\frac{1}{2}\left(\frac{M}{2}\right)^{2} X_{a} X_{a}+\frac{1}{2} M^{2} X_{i} X_{i}-\frac{3 M}{4} i \Theta \gamma_{123} \Theta\right] \\
V_{1} & =-M i \varepsilon_{i j k} \operatorname{tr} X_{i} X_{j} X_{k}-\operatorname{tr} \Theta \gamma_{I}\left[X_{I}, \Theta\right] \\
V_{2} & =-\frac{1}{4} \operatorname{tr}\left[X_{I}, X_{J}\right]\left[X_{I}, X_{J}\right] .
\end{aligned}
$$

The degrees of freedom are: 


\begin{tabular}{|l|cl|c|c|c|}
\hline field & \multicolumn{2}{|l|}{ notation } & mass & modes & symmetry \\
\hline light scalars & $X_{a}$ & $(a=1, \ldots, 6)$ & $M / 2$ & $a_{a}, a_{a}^{\dagger}$ & $\mathrm{SO}(6)$ \\
heavy scalars & $X_{i}$ & $(i=1, \ldots, 3)$ & $M$ & $b_{i}, b_{i}^{\dagger}$ & $\mathrm{SO}(3)$ \\
Majorana spinor & $\Theta_{\alpha}$ & $(\alpha=1, \ldots, 16)$ & $3 M / 4$ & $c_{\alpha}, c_{\alpha}^{\dagger}$ & $\mathrm{SO}(9)$ \\
\hline
\end{tabular}

The "mass parameter" $M$ is in fact dimensionless ${ }^{4}$ and will serve as (inverse) coupling constant in perturbation theory below.

In (2.1) we used the index $I=(a, i)$ to refer to all scalars at once. All fields $\left[X_{a}(t)\right]_{r s}$ etc are function of time and take values in the Lie algebra of the gauge group $\mathrm{SU}(N), r=$ $1, \ldots, N^{2}-1$. In the fermionic sector we work in a representation with charge conjugation matrix equal to unity and with real and symmetric Euclidean Dirac matrices $\left(\gamma_{I}\right)_{\alpha \beta}$.

For the quantization, we introduce the following creation and annihilation operators

$$
\begin{aligned}
& P_{a}=\sqrt{\frac{M}{4}}\left(a_{a}+a_{a}^{\dagger}\right) \quad P_{i}=\sqrt{\frac{M}{2}}\left(b_{i}+b_{i}^{\dagger}\right) \quad \Theta_{\alpha}=c_{\alpha}+c_{\alpha}^{\dagger} \\
& X_{a}=\frac{i}{\sqrt{M}}\left(a_{a}-a_{a}^{\dagger}\right) \quad X_{i}=\frac{i}{\sqrt{2 M}}\left(b_{i}-b_{i}^{\dagger}\right) \quad \text { with } \begin{array}{c}
c=\Pi^{-} \Theta \\
c^{\dagger}=\Pi^{+} \Theta
\end{array}
\end{aligned}
$$

where $\Pi^{ \pm}:=\frac{1}{2}\left(\mathbf{1} \pm i \gamma_{123}\right)$. They satisfy the canonical (anti-)commutation relations

$$
\begin{aligned}
{\left[\left(a_{a}\right)_{r s},\left(a_{b}^{\dagger}\right)_{t u}\right] } & =\delta_{a b}\left(\delta_{s t} \delta_{r u}-\frac{1}{N} \delta_{r s} \delta_{t u}\right), \\
{\left[\left(b_{i}\right)_{r s},\left(b_{j}^{\dagger}\right)_{t u}\right] } & =\delta_{i j}\left(\delta_{s t} \delta_{r u}-\frac{1}{N} \delta_{r s} \delta_{t u}\right), \\
\left\{\left(c_{\alpha}\right)_{r s},\left(c_{\beta}^{\dagger}\right)_{t u}\right\} & =\frac{1}{2} \Pi_{\alpha \beta}^{-}\left(\delta_{s t} \delta_{r u}-\frac{1}{N} \delta_{r s} \delta_{t u}\right) .
\end{aligned}
$$

The free Hamiltonian now reads

$$
H_{0}=\operatorname{tr}\left[\frac{M}{2} a_{a}^{\dagger} a_{a}+M b_{i}^{\dagger} b_{i}+\frac{3 M}{2} c_{\alpha}^{\dagger} c_{\alpha}\right]
$$

Physical states are constrained to be gauge invariant and are given by traces over words in the creation operators

$$
|\psi\rangle=\operatorname{tr}\left(a_{a_{1}}^{\dagger} b_{i_{2}}^{\dagger} c_{\alpha_{3}}^{\dagger} \ldots\right) \operatorname{tr}(\ldots) \ldots|0\rangle \text {. }
$$

In the planar limit we concentrate on single trace states. The number of oscillators in the trace is called the length of a state. It is common to visualize a state of length $L$ as a "spin-chain" with $L$ sites, where every spin orientation corresponds to a particular oscillator. In the case of the full PWMT, the spins are elements of the 17-dimensional module $\operatorname{span}\left\{a_{a}^{\dagger}, b_{i}^{\dagger}, c_{\alpha}^{\dagger}\right\}$ of $\mathfrak{s u}(4 \mid 2)$. In the following, however, we will be interested only in states that are built from fields of a certain $\mathfrak{s u}(2)$ subsector. In order to define this subsector it is convenient to relabel the $\mathfrak{s o}(6)$ fields according to

$$
Z^{\dagger}=\frac{1}{\sqrt{2}}\left(a_{1}^{\dagger}+i a_{2}^{\dagger}\right), \bar{Z}^{\dagger}=\frac{1}{\sqrt{2}}\left(a_{1}^{\dagger}-i a_{2}^{\dagger}\right), Z=\frac{1}{\sqrt{2}}\left(a_{1}-i a_{2}\right), \bar{Z}=\frac{1}{\sqrt{2}}\left(a_{1}+i a_{2}\right) .
$$

\footnotetext{
${ }^{4}$ In terms of M-theoretic quantities it is given by $M=\frac{\mu l_{P}^{2}}{6 R}$, where $\mu$ is the parameter of the plane-wave background, $l_{P}$ is the eleven-dimensional Planck length and $R$ is the radius of the compactified eleventh dimension.
} 
and analogously for $W^{\dagger}=\frac{1}{\sqrt{2}}\left(a_{3}^{\dagger}+i a_{4}^{\dagger}\right)$ and $Y^{\dagger}=\frac{1}{\sqrt{2}}\left(a_{5}^{\dagger}+i a_{6}^{\dagger}\right)$. The only non-vanishing commutators are

$$
\left[(Z)_{r s},\left(Z^{\dagger}\right)_{t u}\right]=\left[(\bar{Z})_{r s},\left(\bar{Z}^{\dagger}\right)_{t u}\right]=\delta_{s t} \delta_{r u}-\frac{1}{N} \delta_{r s} \delta_{t u}
$$

and identical formulas for $W^{\dagger}$ and $Y^{\dagger}$. The mentioned $\mathfrak{s u}(2)$ subsector is spanned by $Z^{\dagger}$ and $W^{\dagger}$. A typical state is given by

$$
\operatorname{tr}\left(Z^{\dagger} Z^{\dagger} W^{\dagger} Z^{\dagger} Z^{\dagger} Z^{\dagger} W^{\dagger} Z^{\dagger} \ldots\right)|0\rangle \widehat{=}|\uparrow \uparrow \downarrow \uparrow \uparrow \uparrow \downarrow \uparrow \ldots\rangle
$$

It is customary to call $W^{\dagger}$ an "impurity" within the "background fields" $Z^{\dagger}$. In the spinchain language $W^{\dagger}$ refers to the excitation of a "magnon". Both fields can be distinguished by a $\mathrm{U}(1)_{R}$ charge $J$ associated to rotations in the 1-2-plane of the $\mathfrak{s o}(6)$ field space:

\begin{tabular}{|c|c|c|c|c|c|}
\hline field & $Z^{\dagger}$ & $\bar{Z}^{\dagger}$ & $Z$ & $\bar{Z}$ & others \\
\hline$J$ & +1 & -1 & -1 & +1 & 0 \\
\hline
\end{tabular}

The R-charge is additive and hence $J$ counts the number of $Z^{\dagger}$ 's in an $\mathfrak{s u}(2)$ state.

Later on we will need the parity conjugate of (single-trace) states, which is defined as the state with reversed order of fields multiplied by $(-1)^{\text {Length }}$.

\subsection{Perturbation theory to fourth order}

The aim is to construct the effective Hamiltonian $T$ (sometimes referred to as energy operator) for the matrix model. The effective Hamiltonian is defined as an operator similar to the full Hamiltonian, $T=U^{-1} H U$, which does not mix states with different free energies. It is the matrix theory operator that corresponds to the dilatation operator of SYM.

The computation of the effective Hamiltonian $T$ has to be done in perturbation theory for large values of $M$. Note that the interaction terms $V_{1}$ and $V_{2}$ in (2.1) are suppressed by powers of $1 / M^{2}$ and $1 / M^{4}$, respectively, as can be seen by rescaling the fields. In [21] $T$ was derived up to three-loop order. For convenience we repeat the formulas of the perturbation expansion of $T$, now including fourth order, in App. B. We have intentionally refrained from choosing the transformation operator $U$ to be unitary. Though this yields a non-hermitian $T$, it has the essential advantage to produce far less terms that need to be computed. This is important because fourth order is very close to the borderline of the technically infeasible. To perform the actual computation we have developed the highly optimized computer program gemstone which is described in detail in App. A.

While at third order it was still possible to find the non-planar effective Hamiltonian 21], at fourth order this is impossible with todays technology. And actually our interest just concerns the planar part of $T$. This is because the integrability is only a feature of the planar limit. Also the connection between PWMT and SYM at the non-planar level has already ceased to exist at three-loop.

Note, however, that the formulas for $T$ as given in (B.1) will produce any graph, in particular non-planar ones. It would be extremely nice to have a formalism that just builds planar graphs automatically. In cases where there are no pure creation and pure annihilation 
vertices, this can indeed be achieved by replacing the matrix oscillators by scalar oscillators and their algebra (2.3) by the Cuntz algebra 23]. But the interaction terms of PWMT unfortunately do not meet this requirement.

Therefore the implementation of the perturbation expansion on the computer has to be supplemented by a special algorithm which guarantees the construction of and only of the planar graphs. Simple extensions of the Cuntz algebra approach suffer from an over counting or an omission of some planar graphs. Our algorithm (which introduces structures like "graphs", "components of connection", "domains" etc) is explained in App. A.3.

There is another general problem of taking the planar limit. When just looking at an operator, as opposed to an operator applied to a state, it is in general not possible to extract its planar part. This is because a superficially non-planar looking operator can in fact produce a planar graph when it is applied to a sufficiently short state. This issue is known as wrapping problem 9 .

The resolution of this problem is to split the computation in pieces: a general computation under the assumption that the states are long enough to avoid wrapping, and then as many special cases as there are lengths which are not covered by the general part. At four-loop order wrapping can only occur for states of length less than or equal to four. These cases will be considered in Sec. 2.4 below. For the time being let us assume that the effective Hamiltonian acts only onto states of length greater than four.

A convenient notation for the planar operators was introduced in [4]: Let $P_{k_{1}, k_{2}}$ exchange the oscillators at sites $k_{1}(\bmod L)$ and $k_{2}(\bmod L)$ of a state of length $L$. Then any planar operator that preserves the number of individual flavours - such as the effective Hamiltonian in the $\mathfrak{s u}(2)$ subsector - can be written as a linear combination of objects of the following kind:

$$
\begin{aligned}
\left\{n_{1}, n_{2}, \ldots\right\} & :=\sum_{k=1}^{L} P_{k+n_{1}, k+n_{1}+1} P_{k+n_{2}, k+n_{2}+1} \cdots, \\
\{\} & :=L .
\end{aligned}
$$

There are many trivial relations for these operators such as

$$
\begin{aligned}
\{\ldots, n, n, \ldots\} & =\{\ldots, \ldots\} \\
\{\ldots, n, m, \ldots\} & =\{\ldots, m, n, \ldots\} \quad \text { for }|n-m| \geq 2 \\
\left\{n_{1}, n_{2}, \ldots\right\} & =\left\{n_{1}+m, n_{2}+m, \ldots\right\}
\end{aligned}
$$

as well as the identity

$$
\begin{aligned}
& \{\ldots, \ldots\}+\{\ldots, n \pm 1, n, \ldots\}+\{\ldots, n, n \pm 1, \ldots\} \\
& -\{\ldots, n, \ldots\}-\{\ldots, n \pm 1, \ldots\}-\{\ldots, n, n \pm 1, n, \ldots\}=0
\end{aligned}
$$

which only holds in the $\mathfrak{s u}(2)$ subsector and expresses the fact that two spins cannot be placed completely antisymmetric onto three sites.

Now, our strategy to determine the planar effective Hamiltonian is the following. We start from an ansatz for $T_{8}$ in terms of the permutation operators (2.9) with free coefficients 
and apply this ansatz to some particular states. The coefficients are then determined by applying the whole perturbation expansion (B.8) to the same states using gemstone and matching the results.

Specifically we use the following ansatz for the planar four-loop effective Hamiltonian

$$
\begin{aligned}
T_{8} & =c_{1}\{\}+c_{2}\{0\}+c_{3}\{0,1\}+c_{4}\{1,0\} \\
& +c_{5}\{0,2\}+c_{6}\{0,1,2\}+c_{7}\{2,1,0\}+c_{8}\{0,2,1\}+c_{9}\{1,0,2\}+c_{10}\{1,0,2,1\} \\
& +c_{11}\{0,3\}+c_{12}\{0,1,3\}+c_{13}\{0,3,2\}+c_{14}\{1,0,3\}+c_{15}\{0,2,3\} \\
& +c_{16}\{0,1,2,3\}+c_{17}\{3,2,1,0\}+c_{18}\{0,2,1,3\}+c_{19}\{1,0,3,2\} \\
& +c_{20}\{0,1,3,2\}+c_{21}\{0,3,2,1\}+c_{22}\{1,0,2,3\}+c_{23}\{2,1,0,3\} \\
& +c_{24}\{0,2,1,3,2\}+c_{25}\{2,1,0,3,2\}+c_{26}\{1,0,2,1,3\}+c_{27}\{1,0,3,2,1\} \\
& +c_{28}\{1,0,2,1,3,2\}+c_{29}\{2,1,0,3,2,1\} .
\end{aligned}
$$

This ansatz is capable of describing any planar operator which acts locally as permutation on five adjacent fields. In fact it is even too general for the effective Hamiltonian which possesses some properties that could actually be used to reduce the number of free coefficients. However, we are not going to make use of these properties in order to have a consistency check for our computation. The price for this is just a marginally increased computation time.

Firstly we could discard all terms which consist of more than 4 elementary permutations. This is because any transposition corresponds to a four-point vertex in the language of Feynman diagrams, and at four-loop order there are at most four of these vertices ${ }^{5}$. Hence, one could set

$$
c_{24}=\ldots=c_{29}=0
$$

right from the beginning leaving just 23 coefficients. The second property is the conservation of parity, which implies that the effective Hamiltonian has to be invariant under the transformation

$$
\left\{n_{1}, \ldots, n_{r}\right\} \rightarrow\left\{-n_{1}, \ldots,-n_{r}\right\} .
$$

This would yield the following further relations

$$
c_{3}=c_{4} \quad c_{6}=c_{7} \quad c_{8}=c_{9} \quad c_{12}=c_{13} \quad c_{14}=c_{15} \quad c_{18}=c_{19} \quad c_{20}=c_{21} \quad c_{22}=c_{23}
$$

such that there are actually only 15 independent coefficients. On the other hand we cannot assume hermiticity of (2.12) since we explicitly destroy the hermiticity by using a nonunitary similarity transformation. But we just note that hermitian conjugation acts as

$$
\left\{n_{1}, \ldots, n_{r}\right\} \rightarrow\left\{n_{r}, \ldots, n_{1}\right\}
$$

Now we need to find an appropriate state, where the effective Hamiltonian should be applied to. The largest number $m$ of coefficients that are fixed by some state of length $L$ is given in the following table, together with a sample chain:

\footnotetext{
${ }^{5}$ This is the Feynman diagrammatic structural constraint mentioned in the introduction, which entered the analysis of $[8]$.
} 


\begin{tabular}{|c|c|l|}
\hline$L$ & $m$ & possible initial chain \\
\hline 6 & 4 & $($ ZZWZWW) \\
7 & 5 & $($ ZZWZZWW) \\
8 & 10 & (ZZZWZWWW) \\
9 & 14 & (ZZZZWZWWW) \\
10 & 24 & (ZZZZWWZWWW) \\
11 & 29 & (ZZZZZWWZWWW) \\
\hline
\end{tabular}

Hence a single initial state with eleven (or more) elementary fields is indeed sufficient to fix all 29 parameters of the ansatz (2.12) at once! A similar analysis for the constrained ansatz with 15 independent coefficients shows that one would have to use a state of length ten.

We chose the initial state

$$
\mid \text { in }\rangle=\operatorname{tr}\left(Z^{\dagger} Z^{\dagger} Z^{\dagger} Z^{\dagger} Z^{\dagger} W^{\dagger} W^{\dagger} Z^{\dagger} W^{\dagger} W^{\dagger} W^{\dagger}\right)|0\rangle
$$

for our computation and apply both the ansatz (2.12) and the whole sequence of vertices (B.8). The result of the latter calculation is given in (A.11) and fixes all coefficients uniquely to

$$
\begin{aligned}
T_{8}=\frac{N^{4}}{M^{11}}[ & -22719\{\}+33143\{0\}-5628(\{0,1\}+\{1,0\})-1044\{0,2\} \\
& +984(\{0,1,2\}+\{2,1,0\})+384\{0,2,1\}-416\{1,0,2\}-28\{1,0,2,1\} \\
& -32\{0,3\}+60(\{0,1,3\}+\{0,3,2\})+4(\{1,0,3\}+\{0,2,3\}) \\
& -80(\{0,1,2,3\}+\{3,2,1,0\})+24(\{0,2,1,3\}+\{1,0,3,2\}) \\
& -32(\{0,1,3,2\}+\{0,3,2,1\})+24(\{1,0,2,3\}+\{2,1,0,3\})] .
\end{aligned}
$$

Note that the requirements (2.13) and (2.15) are satisfied. Also note that the sum of all coefficients is zero. This implies that all states which are composed entirely of $Z^{\dagger}$ 's or entirely of $W^{\dagger}$ 's or which contain only a single oscillator of the other kind have vanishing energy shift. This accounts for the fact that these states are perturbatively protected [24].

We will now switch to SYM-language: By a simple rescaling

$$
D:=\frac{2}{M} T
$$

the effective Hamiltonian turns into the dilatation operator $D$ and the eigenvalues are now called conformal dimensions $\Delta$ instead of energies. The dictionary is given in the following table:

\begin{tabular}{|l|ll|}
\hline \multicolumn{1}{|c|}{ PWMT } & \multicolumn{1}{c|}{ SYM } \\
\hline effective Hamiltonian $T$ & \multicolumn{1}{c|}{ dilatation operator $D$} \\
energy $E$ & \multicolumn{2}{c|}{ conformal dimension $\Delta$} \\
free energy $E_{0}$ & bare conformal dimension $\Delta_{0}$ \\
energy shift $\delta E$ & \multicolumn{2}{|c|}{ anomalous dimension $\delta \Delta$} \\
$E_{0}\left(a^{\dagger}\right)=M / 2$ & $\Delta_{0}(\phi)=1 \quad$ (scalars) \\
$E_{0}\left(b^{\dagger}\right)=M$ & $\Delta_{0}(F)=2$ & (field strength) \\
$E_{0}\left(c^{\dagger}\right)=3 M / 4$ & $\Delta_{0}(\psi)=3 / 2$ & (fermions) \\
\hline
\end{tabular}


In addition to this we introduce a new notation for the effective coupling. The perturbation expansion of $D$ is organized in powers of

$$
\frac{1}{M} T_{2 k} \propto\left(\frac{N}{M^{3}}\right)^{k}
$$

which we now denote by

$$
\frac{\Lambda}{2}:=\frac{N}{M^{3}}
$$

The connection to SYM at the classical level is then given by [2]

$$
\Lambda=G^{2} N \quad, \quad G=\frac{g_{\mathrm{YM}}}{4 \pi} .
$$

With these definitions we find the planar four-loop dilatation operator of PWMT including lower orders from [21] to be

$$
D(\Lambda)=\sum_{k=0}^{\infty} \Lambda^{k} D_{2 k}
$$

$$
\begin{aligned}
D_{0}= & \{\}, \\
D_{2}= & 2\{\}-2\{0\}, \\
D_{4}= & -15\{\}+19\{0\}-2(\{0,1\}+\{1,0\}), \\
D_{6}= & 187\{\}-259\{0\}+38(\{0,1\}+\{1,0\}) \\
& +4\{0,2\}-4(\{0,1,2\}+\{2,1,0\})-2(\{0,2,1\}-\{1,0,2\}), \\
D_{8}= & -\frac{22719}{8}\{\}+\frac{33143}{8}\{0\}-\frac{1407}{2}(\{0,1\}+\{1,0\})-\frac{261}{2}\{0,2\} \\
& +123(\{0,1,2\}+\{2,1,0\})+48\{0,2,1\}-52\{1,0,2\}-\frac{7}{2}\{1,0,2,1\} \\
& -4\{0,3\}+\frac{15}{2}(\{0,1,3\}+\{0,3,2\})+\frac{1}{2}(\{1,0,3\}+\{0,2,3\}) \\
& -10(\{0,1,2,3\}+\{3,2,1,0\})+3(\{0,2,1,3\}+\{1,0,3,2\}) \\
& -4(\{0,1,3,2\}+\{0,3,2,1\})+3(\{1,0,2,3\}+\{2,1,0,3\}) .
\end{aligned}
$$

\subsection{Hermitization}

In App. B we have set up the perturbation theory in such a way that the effective Hamiltonian is computed from the least number of terms. This, however, has led to a non-hermitian

effective Hamiltonian. Therefore we apply to the result (2.24) another similarity transformation of the form

$$
D^{\prime}(\Lambda)=e^{-\Lambda^{3} A_{3}} e^{-\Lambda^{2} A_{2}} e^{-\Lambda A_{1}} D(\Lambda) e^{\Lambda A_{1}} e^{\Lambda^{2} A_{2}} e^{\Lambda^{3} A_{3}} .
$$


We demand that $D^{\prime}$ is hermitian, parity conserving, of maximal range five and made of at most four elementary permutations. This allows the following operators

$$
\begin{aligned}
A_{1}= & \frac{1}{2}\left(x_{L}+x_{R}+1\right)\{0\} \\
A_{2}= & {\left[-\frac{31}{8}+\frac{19}{4}\left(x_{L}+x_{R}\right)+\frac{1}{2}\left(y_{L}+y_{R}\right)-\frac{1}{2}\left(z_{L}+z_{R}\right)\right]\{0\}+x_{L}\{0,1\}+x_{R}\{1,0\} } \\
A_{3}= & w\{0\}+y_{L}\{0,1\}+y_{R}\{1,0\}+\left[\frac{3}{4}-x_{L}-x_{R}\right]\{0,2\} \\
& +\left[-\frac{3}{4}+2 x_{L}-\frac{1}{2}\left(z_{L}+z_{R}\right)\right]\{0,1,2\}+\left[-\frac{3}{4}+2 x_{R}-\frac{1}{2}\left(z_{L}+z_{R}\right)\right]\{2,1,0\} \\
& +z_{L}\{0,2,1\}+z_{R}\{1,0,2\}
\end{aligned}
$$

where $w, x_{L}, x_{R}, y_{L}, y_{R}, z_{L}, z_{R} \in \mathbb{R}$ are arbitrary. The result of this transformation is

$$
\begin{aligned}
D_{0}^{\prime}= & \{\} \\
D_{2}^{\prime}= & 2\{\}-2\{0\} \\
D_{4}^{\prime}= & -15\{\}+19\{0\}-2(\{0,1\}+\{1,0\}), \\
D_{6}^{\prime}= & 187\{\}-259\{0\}+38(\{0,1\}+\{1,0\})+4\{0,2\}-4(\{0,1,2\}+\{2,1,0\}), \\
D_{8}^{\prime}= & -\frac{22719}{8}\{\}+\frac{33127+32 \alpha}{8}\{0\}-\frac{1403+8 \alpha}{2}(\{0,1\}+\{1,0\})-\frac{259+4 \alpha}{2}\{0,2\} \\
& +(122+2 \alpha)(\{0,1,2\}+\{2,1,0\})-(3-2 \alpha)(\{0,2,1\}+\{1,0,2\})-\frac{5+4 \alpha}{2}\{1,0,2,1\} \\
& -4\{0,3\}+4(\{0,1,3\}+\{0,3,2\}+\{1,0,3\}+\{0,2,3\}) \\
& -10(\{0,1,2,3\}+\{3,2,1,0\})-(2+2 \alpha)(\{0,2,1,3\}+\{1,0,3,2\}) \\
& -\alpha(\{0,1,3,2\}+\{0,3,2,1\}+\{1,0,2,3\}+\{2,1,0,3\})
\end{aligned}
$$

where the parameter $\alpha$ depends only on the following particular combination

$$
\alpha=\frac{1}{2}\left(x_{L}+x_{R}\right)^{2}+z_{L}-z_{R} .
$$

It does not influence the conformal dimensions and may therefore be set to any value. This ambiguity accounts for the freedom of making a unitary change of basis in the space of states.

\subsection{Short states - wrapping issues}

We still need to consider the special cases which have been excluded in the previous computation. This concerns all states of length less than or equal to four. Out of the total number of 13 states of this kind only the following two may acquire an anomalous dimension, cf. discussion below Eq. (2.18):

$$
\begin{aligned}
& |Z W Z W\rangle=\operatorname{tr} Z^{\dagger} W^{\dagger} Z^{\dagger} W^{\dagger}|0\rangle, \\
& |Z Z W W\rangle=\operatorname{tr} Z^{\dagger} Z^{\dagger} W^{\dagger} W^{\dagger}|0\rangle .
\end{aligned}
$$

We apply the whole perturbation expansion (B.8) onto these states. The raw results are given in (A.9), (A.9). Converted to the language of the dilatation operator we find

$$
\begin{aligned}
& D_{8}|Z W Z W\rangle=-11028|Z W Z W\rangle+11028|Z Z W W\rangle, \\
& D_{8}|Z Z W W\rangle=+5514|Z W Z W\rangle-5514|Z Z W W\rangle .
\end{aligned}
$$


Let us now see whether this result could follow from the general expression for the effective Hamiltonian $T_{8}$ by any natural description of how to interpret the action of five spin interaction terms on a state of length four: One could either declare the interactions to wrap around the state, i.e. identify site 5 with site 1 . This yields the mixing matrix

$$
\frac{1}{4}\left(\begin{array}{ll}
-43486 & +43486 \\
+21743 & -21743
\end{array}\right)
$$

in disagreement with Eq. (2.31). Alternatively one could simply drop the five spin interaction terms, leading to

$$
\frac{1}{4}\left(\begin{array}{ll}
-43646 & +43710 \\
+21855 & -21791
\end{array}\right)
$$

again in disagreement with Eq. (2.31). We hence conclude that there appears to be no "natural" extension of the long-range form of $D_{8}$ to short states.

This completes the computation of the PWMT-dilatation operator and we will investigate its properties in the next section.

\section{Properties of the PWMT-dilatation operator}

Having obtained the PWMT-dilatation operator, an immediate question is whether it is equivalent to the four-loop proposal of the SYM-dilatation operator given by Beisert, Dippel and Staudacher [8] or alternatively to the spin-chain Hamiltonian of Inozemtsev [10, 7]. In order to make contact to these models we consider a renormalization of the coupling constant $\Lambda$ in order to (naively) reach a thermodynamic scaling property known to exist in the dual string theory ("BMN scaling"). Rewritten in terms of commuting permutations we see, however, that the PWMT-dilatation operator is inequivalent to both of these models. Since the Beisert-Dippel-Staudacher Hamiltonian is the unique SU(2) Hamiltonian which is integrable and obeys BMN scaling up to four loops [8] (the Inozemtsev Hamiltonian violates BMN scaling [7]), the PWMT-dilatation operator must either break integrability or BMN scaling or both. As an indication of integrability we display the degeneracies in the four-loop spectrum and present higher commuting charges. A proof of the breakdown of BMN scaling and deeper insights into the integrable structure of the model will be given in the next section by means of the underlying perturbative asymptotic Bethe ansatz.

\subsection{Renormalization and naive BMN scaling}

Let us consider states carrying two excitations $W^{\dagger}$ among $J$ excitations $Z^{\dagger}$, i.e. a spin-chain of length $L=J+2$ with two magnons:

$$
\left|\mathcal{O}_{l}\right\rangle:=\operatorname{tr} W^{\dagger}\left(Z^{\dagger}\right)^{l-1} W^{\dagger}\left(Z^{\dagger}\right)^{L-1-l}|0\rangle=\left|\mathcal{O}_{L-l}\right\rangle \quad \text { for } 1 \leq l \leq L-1 .
$$

The plane-wave string/gauge theory correspondence predicts a scaling dimension of these states in the full four-dimensional $\mathcal{N}=4$ super Yang-Mills model of the all-loop form (with 
the $\mathrm{U}(1)_{R}$ charge $\left.J=L-2\right)$

$$
\Delta^{\mathrm{BMN}}(n, J)=J+2 \sqrt{1+\Lambda \frac{16 \pi^{2} n^{2}}{J^{2}}} \quad \text { with } n \in \mathbb{N}
$$

in the limit $J \rightarrow \infty$. This expression displays BMN scaling behavior, i.e. in the limit $J, N \rightarrow \infty$, with $N / J^{2}$ held fixed, the effective loop counting parameter $\Lambda^{\prime}:=\Lambda / J^{2}$ arises. So let us check whether this scaling behavior is realized in PWMT by simply applying the dilatation operator (2.27) to the set of the above two-magnon states. For the time being we consider only those states where the magnons are further apart than the range of the dilatation operator, i.e. the dilatation operator does not act on both magnons at the same time. Technically speaking we restrict the action of $D_{2 k}$ to states $\left|\mathcal{O}_{l}\right\rangle$ with $k<l<L-k$ and find

$$
\begin{aligned}
D_{2}^{\prime}\left|\mathcal{O}_{l}\right\rangle & =+8\left|\mathcal{O}_{l}\right\rangle-4\left|\mathcal{O}_{l \pm 1}\right\rangle \\
D_{4}^{\prime}\left|\mathcal{O}_{l}\right\rangle & =-52\left|\mathcal{O}_{l}\right\rangle+30\left|\mathcal{O}_{l \pm 1}\right\rangle-4\left|\mathcal{O}_{l \pm 2}\right\rangle \\
D_{6}^{\prime}\left|\mathcal{O}_{l}\right\rangle & =+612\left|\mathcal{O}_{l}\right\rangle-374\left|\mathcal{O}_{l \pm 1}\right\rangle+76\left|\mathcal{O}_{l \pm 2}\right\rangle-8\left|\mathcal{O}_{l \pm 3}\right\rangle \\
D_{8}^{\prime}\left|\mathcal{O}_{l}\right\rangle & =-\frac{17923}{2}\left|\mathcal{O}_{l}\right\rangle+\frac{22615}{4}\left|\mathcal{O}_{l \pm 1}\right\rangle-1397\left|\mathcal{O}_{l \pm 2}\right\rangle+244\left|\mathcal{O}_{l \pm 3}\right\rangle-20\left|\mathcal{O}_{l \pm 4}\right\rangle
\end{aligned}
$$

where we used the shorthand $\left|\mathcal{O}_{l \pm m}\right\rangle:=\left|\mathcal{O}_{l-m}\right\rangle+\left|\mathcal{O}_{l+m}\right\rangle$. The observation now is that this may be written as a sum of even powers of a discretized second derivative $\partial_{l}^{2}$ which acts as $\partial_{l}^{2}\left|\mathcal{O}_{l}\right\rangle=-2\left|\mathcal{O}_{l}\right\rangle+\left|\mathcal{O}_{l \pm 1}\right\rangle$. One finds

$$
\begin{aligned}
& D_{2}^{\prime}=-4 \partial_{l}^{2} \\
& D_{4}^{\prime}=-4 \partial_{l}^{4}+14 \partial_{l}^{2}, \\
& D_{6}^{\prime}=-\mathbf{8} \partial_{l}^{6}+28 \partial_{l}^{4}-142 \partial_{l}^{2}, \\
& D_{8}^{\prime}=-\mathbf{2 0} \partial_{l}^{8}+84 \partial_{l}^{6}-333 \partial_{l}^{4}+\frac{7767}{4} \partial_{l}^{2} .
\end{aligned}
$$

Now it is easy to determine the spectrum (neglecting the boundary effects of the action of $D^{\prime}$ involving two magnons at the same time). On the Fourier transformed basis

$$
|n\rangle:=\sum_{l=1}^{L-1} e^{2 \pi i n(l-1) / J}\left|\mathcal{O}_{l}\right\rangle \quad(J=L-2)
$$

the dilatation operator becomes diagonal since

$$
\partial_{l}^{2}|n\rangle=\left(-2+e^{2 \pi i n / J}+e^{-2 \pi i n / J}\right)|n\rangle=-4 \sin ^{2}\left(\frac{\pi n}{J}\right)|n\rangle \stackrel{J \rightarrow \infty}{\longrightarrow}-\frac{4 \pi^{2} n^{2}}{J^{2}}|n\rangle .
$$

On the other hand, if one expands the square root formula (3.2) in a series in $\Lambda$

$$
\begin{aligned}
\Delta^{\mathrm{BMN}}(n, J)=J+2 & -4 \Lambda\left(\frac{-4 \pi^{2} n^{2}}{J^{2}}\right)-4 \Lambda^{2}\left(\frac{-4 \pi^{2} n^{2}}{J^{2}}\right)^{2} \\
& -\mathbf{8} \Lambda^{3}\left(\frac{-4 \pi^{2} n^{2}}{J^{2}}\right)^{3}-\mathbf{2 0} \Lambda^{4}\left(\frac{-4 \pi^{2} n^{2}}{J^{2}}\right)^{4}+\ldots
\end{aligned}
$$


one observes, that these values are exactly produced by the highest derivatives in $D^{\prime}$. The lower order derivatives, however, spoil the BMN scaling behavior (3.2). But this can be restored by a redefinition of the coupling constant. If we set

$$
\Lambda=\Lambda_{r}+\frac{7}{2} \Lambda_{r}^{2}-11 \Lambda_{r}^{3}+\frac{1257}{16} \Lambda_{r}^{4}+\mathcal{O}\left(\Lambda_{r}^{5}\right)
$$

then the dilatation operator expanded in $\Lambda_{r}$ is simply given by clean lattice laplacians of ascending order

$$
D_{r, 2}^{\prime}=-4 \partial_{l}^{2} \quad, \quad D_{r, 4}^{\prime}=-4 \partial_{l}^{4} \quad, \quad D_{r, 6}^{\prime}=-8 \partial_{l}^{6} \quad, \quad D_{r, 8}^{\prime}=-20 \partial_{l}^{8}
$$

and hence in accordance with BMN scaling.

We wish to stress, that the precise series of coefficients $4,4,8,20$ of the plane-wave string formula (3.2), (3.7) is an inherent property of PWMT and cannot be altered by any perturbative redefinition of $\Lambda$. Only the derivatives of non-highest degree could be eliminated, and demanding their absence as a necessary prerequisite for BMN scaling uniquely fixes the renormalization (3.8). Any differently renormalized coupling constant would prevent BMN scaling immediately.

Hence, from now on we will work with the dilatation operator obtained from (2.27) by redefining $\Lambda$ according to the naive BMN scaling prescription (3.8):

$$
\begin{aligned}
D_{r, 0}^{\prime}= & \{\}, \\
D_{r, 2}^{\prime}= & 2\{\}-2\{0\}, \\
D_{r, 4}^{\prime}= & -8\{\}+12\{0\}-2(\{0,1\}+\{1,0\}), \\
D_{r, 6}^{\prime}= & 60\{\}-104\{0\}+24(\{0,1\}+\{1,0\})+4\{0,2\}-4(\{0,1,2\}+\{2,1,0\}), \\
D_{r, 8}^{\prime}= & -573\{\}+(1079+4 \alpha)\{0\}-(283+4 \alpha)(\{0,1\}+\{1,0\})-\frac{175+4 \alpha}{2}\{0,2\} \\
& +(80+2 \alpha)(\{0,1,2\}+\{2,1,0\})-(3-2 \alpha)(\{0,2,1\}+\{1,0,2\})-\frac{5+4 \alpha}{2}\{1,0,2,1\} \\
& -4\{0,3\}+4(\{0,1,3\}+\{0,3,2\}+\{1,0,3\}+\{0,2,3\}) \\
& -10(\{0,1,2,3\}+\{3,2,1,0\})+(2+2 \alpha)(\{0,2,1,3\}+\{1,0,3,2\}) \\
& -\alpha(\{0,1,3,2\}+\{0,3,2,1\}+\{1,0,2,3\}+\{2,1,0,3\})
\end{aligned}
$$

One has to keep in mind, that these considerations only naively confirm the BMN scaling behavior of the renormalized dilatation operator, as the neglected boundary terms could (and actually do) ruin this scaling. Clearly the contact interactions of two magnons will be suppressed by a factor of $1 / J$ with respect to the generic terms, however, such a suppression does not suffice to implement BMN scaling at higher orders in perturbation theory. This will be seen in Sec. 4.4. 


\subsection{Distinction to Inozemtsev and Beisert-Dippel-Staudacher spin-chains}

The comparison is most easily done in the language of commuting permutations, which was used in [7]. Our PWMT-dilatation operator then reads

$$
\begin{aligned}
D_{r, 2}^{\prime}= & 2 L-2 \sum_{i} P_{i, i+1} \\
D_{r, 4}^{\prime}= & -6 L+8 \sum_{i} P_{i, i+1}-2 \sum_{i} P_{i, i+2} \\
D_{r, 6}^{\prime}= & 40 L-56 \sum_{i} P_{i, i+1}+16 \sum_{i} P_{i, i+2}+4 \sum_{i}\left(P_{i, i+2} P_{i+1, i+3}-P_{i, i+3} P_{i+1, i+2}\right), \\
D_{r, 8}^{\prime}= & -363 L+522 \sum_{i} P_{i, i+1}-\frac{415}{3} \sum_{i} P_{i, i+2}-\frac{41}{3} \sum_{i} P_{i, i+3}+\mathbf{6} \sum_{i} P_{i, i+4} \\
& -\frac{71}{6} \sum_{i} P_{i, i+1} P_{i+2, i+3}+\frac{217}{3} \sum_{i} P_{i, i+3} P_{i+1, i+2}-\frac{147}{2} \sum_{i} P_{i, i+2} P_{i+1, i+3} \\
& +\frac{\mathbf{1 6}}{\mathbf{3}} \sum_{i}\left(P_{i, i+3} P_{i+2, i+4}+P_{i, i+2} P_{i+1, i+4}+P_{i, i+3} P_{i+1, i+4}\right. \\
& \left.\quad-P_{i, i+4} P_{i+2, i+3}-P_{i, i+4} P_{i+1, i+2}-P_{i, i+4} P_{i+1, i+3}\right) .
\end{aligned}
$$

For the four-loop contribution we have committed ourselves to the choice of $\alpha=-2 / 3$ for the free parameter of unitary transformations in order to obtain the same coefficient for all terms in the last two lines of (3.11). Comparing to the results obtained in [7] it is clear that we are not dealing with the Inozemtsev spin-chain here, as that would predict the five spin interaction terms with different coefficients, namely

$$
\begin{aligned}
D_{8, \text { Inozemtsev }}= & 4 \sum_{i} P_{i, i+4}+4 \sum_{i}\left(P_{i, i+3} P_{i+2, i+4}+P_{i, i+2} P_{i+1, i+4}+P_{i, i+3} P_{i+1, i+4}\right. \\
& \left.-P_{i, i+4} P_{i+2, i+3}-P_{i, i+4} P_{i+1, i+2}-P_{i, i+4} P_{i+1, i+3}\right)+\ldots
\end{aligned}
$$

Curiously our five spin interaction terms do agree with the proposed four-loop dilation operator of $\mathcal{N}=4$ super Yang-Mills theory [8]. However, they differ in the two, three and four spin interaction terms. The precise discrepancy to the Beisert-Dippel-Staudacher dilatation operator $D_{8}^{\mathrm{BDS}}$ is

$$
\begin{aligned}
D_{r, 8}^{\prime}-D_{8}^{\mathrm{BDS}}=585[ & -L+2 \sum_{i} P_{i, i+1}-\sum_{i} P_{i, i+2}+\sum_{i} P_{i, i+3} \\
& \left.-\frac{1}{2} \sum_{i} P_{i, i+1} P_{i+2, i+3}-\sum_{i} P_{i, i+3} P_{i+1, i+2}+\frac{1}{2} \sum_{i} P_{i, i+2} P_{i+1, i+3}\right] .
\end{aligned}
$$

Thus it is clear that we are facing a model, which is neither of the Inozemtsev nor of the "novel" Beisert-Dippel-Staudacher type. Nevertheless, the PWMT-dilatation operator 
describes a perturbatively integrable long range spin-chain as we will indicate in the next subsection and confirm afterwards by deriving appropriate Bethe equations. ${ }^{6}$

\subsection{Degenerate spectrum and higher charges}

In Tab. 1 we list the (renormalized) four-loop anomalous dimensions $\delta \Delta_{r}$ of all $\mathfrak{s u}(2)-$ multiplets with states of length $L \leq 9$. Apart form the length which is equal to the bare dimension $\Delta_{0}$, multiplets are labeled by the magnon number $M$. Alternatively one could use the $\mathfrak{s u}(2)$ Dynkin label $a=L-2 M$. Every multiplet, which is specified by the pair $(L, M)$, is realized with a certain multiplicity $m$. All of these multiplets can mix among each other. Since the dilatation operator commutes with the parity operator, it is possible to disentangle the multiplets such that all states within one multiplet have equal parity $p$. This has been done in Tab. 1 and is indicated by $m^{p}$.

The crucial observation is that whenever there are multiplets of positive and negative parity with equal $(L, M)$-labels, then they form pairs of degenerate conformal dimension, which have been highlighted in the table through a bold font. This degeneracy is ascribed to a conserved charge and was the original indication of integrability [4]. In fact integrability implies the existence of a set $\left\{Q_{i}\right\}$ of as many conserved charges as there are degrees of freedom:

$$
\left[D_{r}^{\prime}, Q_{i}\right]=0 \quad, \quad\left[Q_{i}, Q_{j}\right]=0 \quad \forall i, j \in\{3,4,5, \ldots\} .
$$

Here we have adopted the usual enumeration of the so-called higher charges starting from $i=3$, cf. 4.1. All charges are Hermite an. Charges with even (odd) index (anti-)commute with the parity operator. Therefore the odd-indexed charges are responsible for the degeneracy of the parity pairs.

The charges have a perturbative expansion

$$
Q_{i}\left(\Lambda_{r}\right)=\sum_{k=1}^{\infty} \Lambda_{r}^{k-1} Q_{i, 2 k}
$$

and can be written in terms of permutations (2.9). The higher the charge the wider its range: $Q_{i, 2 k}$ acts simultaneous on $i+k-1$ adjacent spins. In App. Clwe give $Q_{3}$ and $Q_{4}$ up to $k=4$.

Let us go back to Tab. 1 1 and look at the state with labels $(L, M)=(4,2)$. It is given by $\operatorname{tr}\left[Z^{\dagger}, W^{\dagger}\right]^{2}|0\rangle$ and is in fact a $\mathfrak{s u}(2,2 \mid 4)$-descendant of the Konishi operator $\operatorname{tr} a_{a}^{\dagger} a_{a}^{\dagger}|0\rangle=$ $\operatorname{tr}\left[Z^{\dagger} \bar{Z}^{\dagger}+W^{\dagger} \bar{W}^{\dagger}+Y^{\dagger} \bar{Y}^{\dagger}\right]|0\rangle$. The anomalous dimension of Konishi is known up to threeloop [4, 17, 18, 19. As we have done the explicit computation (2.31), we dare to predict

\footnotetext{
${ }^{6}$ Looking through the literature, we find an old proposal for the SYM-dilatation operator in Ref. [4] which is equivalent to the PWMT one. Formula (F.3) of [4] with the replacement $\alpha \rightarrow-1 / 4$ and $\beta \rightarrow \alpha+1$ matches exactly our above finding (3.10). In [4] integrability was implemented but the implications of BMN scaling were not fully exploited yet. From this result, one could principally already infer the integrability of PWMT and the failure of BMN scaling.
} 


\begin{tabular}{|c|c|c|c|}
\hline$L$ & $M$ & $m^{p}$ & $\delta \Delta_{r} / \Lambda_{r}$ \\
\hline 2 & 0 & $1^{+}$ & 0 \\
\hline 3 & 0 & $1^{-}$ & 0 \\
\hline \multirow[t]{2}{*}{$\overline{4}$} & 0 & $1^{+}$ & 0 \\
\hline & 2 & $1^{+}$ & $12-48 \Lambda_{r}+336 \Lambda_{r}^{2}-12771 / 4 \Lambda_{r}^{3}$ \\
\hline \multirow[t]{2}{*}{5} & 0 & $1^{-}$ & 0 \\
\hline & 2 & $1^{-}$ & $8-24 \Lambda_{r}+136 \Lambda_{r}^{2}-1024 \Lambda_{r}^{3}$ \\
\hline \multirow[t]{3}{*}{6} & 0 & $1^{+}$ & 0 \\
\hline & 2 & $2^{+}$ & $\begin{array}{l}(10-\sqrt{5})-(34-\sqrt{5}) \Lambda_{r}+\frac{1}{5}(1170-414 \sqrt{5}) \Lambda_{r}^{2}-\frac{1}{5}(10695-4134 \sqrt{5}) \Lambda_{r}^{3} \\
(10+\sqrt{5})-(34+\sqrt{5}) \Lambda_{r}+\frac{1}{5}(1170+414 \sqrt{5}) \Lambda_{r}^{2}-\frac{1}{5}(10695+4134 \sqrt{5}) \Lambda_{r}^{3}\end{array}$ \\
\hline & 3 & $1^{-}$ & $12-36 \Lambda_{r}+252 \Lambda_{r}^{2}-2484 \Lambda_{r}^{3}$ \\
\hline \multirow[t]{4}{*}{7} & 0 & $1^{-}$ & 0 \\
\hline & 2 & $2^{-}$ & $\begin{array}{l}4-6 \Lambda_{r}+37 / 2 \Lambda_{r}^{2}-335 / 4 \Lambda_{r}^{3} \\
12-42 \Lambda_{r}+555 / 2 \Lambda_{r}^{2}-9465 / 4 \Lambda_{r}^{3}\end{array}$ \\
\hline & 3 & $1^{+}$ & $10-30 \Lambda_{\mathrm{r}}+200 \Lambda_{\mathrm{r}}^{2}-3565 / 2 \Lambda_{\mathrm{r}}^{3}$ \\
\hline & & $1^{-}$ & $10-30 \Lambda_{\mathrm{r}}+200 \Lambda_{\mathrm{r}}^{2}-3565 / 2 \Lambda_{\mathrm{r}}^{3}$ \\
\hline \multirow[t]{5}{*}{8} & 0 & $1^{+}$ & 0 \\
\hline & 2 & $3^{+}$ & $\begin{array}{l}3.01-3.32 \Lambda_{r}+7.66 \Lambda_{r}^{2}-27.30 \Lambda_{r}^{3} \\
9.78-29.22 \Lambda_{r}+167.64 \Lambda_{r}^{2}-1256.15 \Lambda_{r}^{3} \\
15.21-59.45 \Lambda_{r}+456.70 \Lambda_{r}^{2}-4430.55 \Lambda_{r}^{3} \\
\end{array}$ \\
\hline & 3 & $1^{+}$ & $8-20 \Lambda_{\mathrm{r}}+112 \Lambda_{\mathrm{r}}^{2}-842 \Lambda_{\mathrm{r}}^{3}$ \\
\hline & & $2^{-}$ & 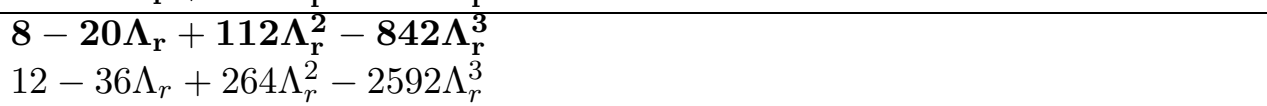 \\
\hline & 4 & $3^{+}$ & $\begin{array}{l}6.49-7.56 \Lambda_{r}+10.22 \Lambda_{r}^{2}+10.25 \Lambda_{r}^{3} \\
10.90-31.76 \Lambda_{r}+249.76 \Lambda_{r}^{2}-2538.27 \Lambda_{r}^{3} \\
22.60-88.68 \Lambda_{r}+636.03 \Lambda_{r}^{2}-5933.98 \Lambda_{r}^{3}\end{array}$ \\
\hline \multirow[t]{6}{*}{9} & 0 & $1^{-}$ & 0 \\
\hline & 2 & $3^{-}$ & $\begin{array}{l}(8-4 \sqrt{2})-(26-17 \sqrt{2}) \Lambda_{r}+\frac{1}{8}(179-993 \sqrt{2}) \Lambda_{r}^{2}-\frac{1}{32}(51376-36103 \sqrt{2}) \Lambda_{r}^{3} \\
8-20 \Lambda_{r}+98 \Lambda_{r}^{2}-639 \Lambda_{r}^{3} \\
(8+4 \sqrt{2})-(26+17 \sqrt{2}) \Lambda_{r}+\frac{1}{8}(179+993 \sqrt{2}) \Lambda_{r}^{2}-\frac{1}{32}(51376+36103 \sqrt{2}) \Lambda_{r}^{3}\end{array}$ \\
\hline & 3 & $3^{+}$ & $\begin{array}{l}6.45-13.18 \Lambda_{\mathrm{r}}+60.73 \Lambda_{\mathrm{r}}^{2}-378.34 \Lambda_{\mathrm{r}}^{3} \\
11.04-33.50 \Lambda_{\mathrm{r}}+231.93 \Lambda_{\mathrm{r}}^{2}-2149.91 \Lambda_{\mathrm{r}}^{3} \\
16.51-55.32 \Lambda_{\mathrm{r}}+383.33 \Lambda_{\mathrm{r}}^{2}-3494.75 \Lambda_{\mathrm{r}}^{3}\end{array}$ \\
\hline & & $3^{-}$ & $\begin{array}{l}6.45-13.18 \Lambda_{\mathrm{r}}+60.73 \Lambda_{\mathrm{r}}^{2}-378.34 \Lambda_{\mathrm{r}}^{3} \\
11.04-33.50 \Lambda_{\mathrm{r}}+231.93 \Lambda_{\mathrm{r}}^{2}-2149.91 \Lambda_{\mathrm{r}}^{3} \\
16.51-55.32 \Lambda_{\mathrm{r}}+383.33 \Lambda_{\mathrm{r}}^{2}-3494.75 \Lambda_{\mathrm{r}}^{3}\end{array}$ \\
\hline & 4 & $1^{+}$ & $10-30 \Lambda_{\mathrm{r}}+220 \Lambda_{\mathrm{r}}^{2}-4185 / 2 \Lambda_{\mathrm{r}}^{3}$ \\
\hline & & $3^{-}$ & $\begin{array}{l}(12-4 \sqrt{3})-18(2-\sqrt{3}) \Lambda_{r}+\frac{1}{2}(456-255 \sqrt{3}) \Lambda_{r}^{2}-\frac{1}{4}(7908-4541 \sqrt{3}) \Lambda_{r}^{3} \\
\mathbf{1 0}-\mathbf{3 0} \boldsymbol{\Lambda}_{\mathbf{r}}+\mathbf{2 2 0} \boldsymbol{\Lambda}_{\mathbf{r}}^{\mathbf{2}}-\mathbf{4 1 8 5} / \mathbf{2} \boldsymbol{\Lambda}_{\mathbf{r}}^{\mathbf{3}} \\
(12+4 \sqrt{3})-18(2+\sqrt{3}) \Lambda_{r}+\frac{1}{2}(456+255 \sqrt{3}) \Lambda_{r}^{2}-\frac{1}{4}(7908+4541 \sqrt{3}) \Lambda_{r}^{3}\end{array}$ \\
\hline
\end{tabular}

Table 1: Particle spectrum in $\mathfrak{s u}(2)$ subsector 
the four-loop value under the assumption of a valid PWMT-SYM-relationship

$$
\begin{aligned}
\delta \Delta_{r} & =12 \Lambda_{r}-48 \Lambda_{r}^{2}+336 \Lambda_{r}^{3}-\frac{12771}{4} \Lambda_{r}^{4} \\
& =\frac{3 \lambda_{\mathrm{YM}}}{4 \pi^{2}}-\frac{3 \lambda_{\mathrm{YM}}^{2}}{16 \pi^{4}}+\frac{21 \lambda_{\mathrm{YM}}^{3}}{256 \pi^{6}}-\frac{12771 \lambda_{\mathrm{YM}}^{4}}{262144 \pi^{8}},
\end{aligned}
$$

where we used (2.22) for the renormalized coupling. It would be extremely nice to test this conjecture through an explicit four-loop computation in the full superconformal $\mathcal{N}=4$ gauge field theory.

\section{Bethe ansatz for plane-wave matrix theory}

In this section we shall deduce the long-range Bethe ansatz allowing for an exact diagonalization of the obtained PWMT-Hamiltonian in the planar limit. We use a technique which was very recently developed in [22] and called "perturbative asymptotic Bethe ansatz". At first we derive the Bethe equations in the two-magnon sector. These are straightforwardly generalized to the full $N$-magnon sector and verified for a number of three and four-magnon states through numeric diagonalization. We then use the Bethe ansatz to solve the complete two-magnon problem, which explicitly reveals terms that violate BMN scaling.

\subsection{Spin-chain language}

For the following discussion we switch the interpretation of the effective PWMT-Hamiltonian $T$, which intermediately became the PWMT-dilatation operator $D_{r}^{\prime}$, a second time. Now, the anomalous piece of the dilatation operator $\delta D_{r}^{\prime}$ is regarded as the Hamiltonian $Q_{2}$ of an $\mathfrak{s u}(2)$ spin-chain

$$
Q_{2}:=\Lambda_{r}^{-1} \delta D_{r}=\Lambda_{r}^{-1}\left(D_{r}-D_{r, 0}\right) .
$$

The spin-chain is in accordance with our previous manner of speaking still given by the single trace $\mathfrak{s u}(2)$ states of PWMT. With notation (4.1) the Hamiltonian joins nicely the set of higher charges $\left\{Q_{i \geq 3}\right\}$. The label $Q_{1}$ is reserved for the spin-chain momentum operator, which in our case is identically to zero due to the cyclicity of the trace. We denote the eigenvalues of $Q_{i}$ by corresponding small letters $q_{i}$. The following table lists equivalent quantities:

\begin{tabular}{|l|l|l|}
\hline \multicolumn{1}{|c|}{ PWMT } & \multicolumn{1}{|c|}{ SYM } & \multicolumn{1}{c|}{ Spin-chain } \\
\hline energy shift operator $\delta T$ & anomalous dilatation operator $\delta D_{r}^{\prime}$ & Hamiltonian $Q_{2}$ \\
energy shift $\delta E$ & anomalous dimension $\delta \Delta_{r}$ & energy $q_{2}$ \\
free energy $E_{0}$ & bare conformal dimension $\Delta_{0}$ & spin-chain length $L$ \\
\hline
\end{tabular}

\subsection{Two-magnon scattering}

The application of the dilatation operator to a spin-chain causes motion and interaction of the magnons. Integrability implies that only two magnons interact at a time. Therefore it is possible to deduce the general case from studying the two-magnon situation. However, 
one must not use the cyclic two-magnon states (3.1) 22] where only the distance of the magnons matters (or alternative their relative momentum). Once we place more magnons onto the spin-chain the position (or momentum) relative to the new insertions becomes important. Thus we need to investigate open two-magnon states, the building blocks for a general state:

$$
\left|\mathcal{O}_{l_{1}, l_{2}}\right\rangle:=\left(Z^{\dagger}\right)^{l_{1}-1} W^{\dagger}\left(Z^{\dagger}\right)^{l_{2}-l_{1}-1} W^{\dagger}\left(Z^{\dagger}\right)^{L-l_{2}}|0\rangle \quad \text { for } 1 \leq l_{1}<l_{2} \leq L-1 .
$$

The dilatation operator mixes all of these $L(L-1) / 2$ states. We superpose them to eigenstates parametrized by two yet undetermined complex variables $p_{1}$ and $p_{2}$

$$
\left|p_{1}, p_{2}\right\rangle:=\sum_{\substack{l_{1}, l_{2}=1 \\ l_{1}<l_{2}}}^{L} a\left(l_{1}, l_{2}, p_{1}, p_{2}\right)\left|\mathcal{O}_{l_{1}, l_{2}}\right\rangle .
$$

This may be regarded as a kind of Fourier transformation, the magnons are now labeled by their (quasi-)momenta $p_{1}$ and $p_{2}$. The Bethe ansatz for $a\left(l_{1}, l_{2}, p_{1}, p_{2}\right)$ resembles an in-coming and a scattered out-going wave ${ }^{7}$

$$
a\left(l_{1}, l_{2}, p_{1}, p_{2}\right)=e^{i\left(p_{1} l_{1}+p_{2} l_{2}\right)} f\left(l_{2}-l_{1}, p_{1}, p_{2}\right)+S\left(p_{2}, p_{1}\right) e^{i\left(p_{1} l_{2}+p_{2} l_{1}\right)} f\left(L-l_{2}+l_{1}, p_{1}, p_{2}\right) .
$$

This ansatz will exactly diagonalize the spin-chain Hamiltonian

$$
Q_{2}\left|p_{1}, p_{2}\right\rangle=q_{2}\left(p_{1}, p_{2}\right)\left|p_{1}, p_{2}\right\rangle .
$$

The key ingredient of the ansatz is the S-matrix $S\left(p_{1}, p_{2}\right)$, which describes the scattering of two magnons and solely determines the eigenvalue of $\left|p_{1}, p_{2}\right\rangle$. In the following we will determine the S-matrix perturbatively up to order $\Lambda_{r}^{3}$

$$
S\left(p_{1}, p_{2}\right)=S_{0}\left(p_{1}, p_{2}\right)+\Lambda_{r} S_{1}\left(p_{1}, p_{2}\right)+\Lambda_{r}^{2} S_{2}\left(p_{1}, p_{2}\right)+\Lambda_{r}^{3} S_{3}\left(p_{1}, p_{2}\right)+\ldots
$$

The function $f\left(l, p_{1}, p_{2}\right)$ takes the special perturbative form

$$
f\left(l, p_{1}, p_{2}\right)=1+\Lambda_{r}^{l} f_{0}\left(l, p_{1}, p_{2}\right)+\Lambda_{r}^{l+1} f_{1}\left(l, p_{1}, p_{2}\right)+\Lambda_{r}^{l+2} f_{2}\left(l, p_{1}, p_{2}\right)+\ldots
$$

and has been introduced to account for boundary effects where the magnons come close to each other. The fact that the range of the spin-chain Hamiltonian depends on the loop order, explains why the order of the leading correction in (4.7) depends on the magnon separation $l=l_{2}-l_{1}$. It turns out that the precise form of the function $f\left(l, p_{1}, p_{2}\right)$ is irrelevant for the energy eigenvalue $q_{2}\left(p_{1}, p_{2}\right)$.

There is another "boundary effect" where the magnons approach either end of the open spin-chain (4.2), i.e. where $l_{1} \approx 1$ or $l_{2} \approx L$. Since the spin-chain Hamiltonian (4.1), (3.10) is not well-defined for open chains we will neglect these cases and assume $l_{1}$ to be large and $l_{2}$ to be small enough. Moreover we may replace the function $f\left(L-l_{2}+l_{1}, p_{1}, p_{2}\right)$ in

\footnotetext{
${ }^{7}$ Notice the reversed order of the arguments in $S$. It will turn out that $S\left(p_{1}, p_{2}\right)=\left[S\left(p_{2}, p_{1}\right)\right]^{-1}$. Also note that the detailed form of our ansatz differs slightly from the one considered in [22].
} 
the scattered piece of the wave function by 1 , if we also assume $L$ to be large enough, because the perturbative corrections will only contribute at order $\Lambda^{L-l_{2}+l_{1}}$. The reason for introducing $f$ at this point lies in the derivation of the Bethe equations from the periodicity conditions, cf. 4.15).

It will turn out that despite these assumptions the wave-function and the eigenvalues are exactly (and correctly) determined and hence the S-matrix, the Bethe equations, and the energy formula as well. Since this is obviously not a rigorous derivation we shall check the result for a number of cases by explicit numerical diagonalization for low values of $L$.

Now, in order to determine the unknown functions of the ansatz (4.4) from demanding the eigenvalue equation (4.5), we apply the spin-chain Hamiltonian to the states (4.3). We do this order by order in $\Lambda_{r}$. The action of the Hamiltonian on $\left|\mathcal{O}_{l_{1}, l_{2}}\right\rangle$ is given by ${ }^{8}$

$$
\begin{aligned}
Q_{2,2}\left|\mathcal{O}_{l_{1}, l_{2}}\right\rangle= & \begin{cases}4\left|\mathcal{O}_{l_{1}, l_{2}}\right\rangle-2\left|\mathcal{O}_{l_{1}-1, l_{2}}\right\rangle-2\left|\mathcal{O}_{l_{1}, l_{2}+1}\right\rangle & \text { for } l_{2}=l_{1}+1 \\
8\left|\mathcal{O}_{l_{1}, l_{2}}\right\rangle-2\left|\mathcal{O}_{l_{1} \pm 1, l_{2}}\right\rangle-2\left|\mathcal{O}_{l_{1}, l_{2} \pm 1}\right\rangle & \text { for } l_{2} \geq l_{1}+2\end{cases} \\
Q_{2,4}\left|\mathcal{O}_{l_{1}, l_{2}}\right\rangle= & \begin{cases}-8\left|\mathcal{O}_{l_{1}, l_{2}}\right\rangle+8\left|\mathcal{O}_{l_{1}-1, l_{2}}\right\rangle+8\left|\mathcal{O}_{l_{1}, l_{2}+1}\right\rangle \\
-2\left|\mathcal{O}_{l_{1}-1, l_{2}-1}\right\rangle-2\left|\mathcal{O}_{l_{1}+1, l_{2}+1}\right\rangle-2\left|\mathcal{O}_{l_{1}-2, l_{2}}\right\rangle-2\left|\mathcal{O}_{l_{1}, l_{2}+2}\right\rangle & \text { for } l_{2}=l_{1}+1 \\
-28\left|\mathcal{O}_{l_{1}, l_{2}}\right\rangle+8\left|\mathcal{O}_{l_{1} \pm 1, l_{2}}\right\rangle+8\left|\mathcal{O}_{l_{1}, l_{2} \pm 1}\right\rangle & \text { for } l_{2}=l_{1}+2 \\
-2\left|\mathcal{O}_{l_{1}-2, l_{2}}\right\rangle-2\left|\mathcal{O}_{l_{1}, l_{2}+2}\right\rangle & \\
-24\left|\mathcal{O}_{l_{1}, l_{2}}\right\rangle+8\left|\mathcal{O}_{l_{1} \pm 1, l_{2}}\right\rangle+8\left|\mathcal{O}_{l_{1}, l_{2} \pm 1}\right\rangle & \\
-2\left|\mathcal{O}_{l_{1} \pm 2, l_{2}}\right\rangle-2\left|\mathcal{O}_{l_{1}, l_{2} \pm 2}\right\rangle & \text { for } l_{2} \geq l_{1}+3\end{cases}
\end{aligned}
$$

and similar more involved expressions for $Q_{2,6}$ and $Q_{2,8}$ which we refrain from stating explicitly here. Due to the extended range of the interaction, we need to consider $k$ special cases for $Q_{2,2 k}$; for $l_{2} \geq l_{1}+k+1$ the formula becomes generic. Clearly the action of the dilatation operator is not diagonal in this basis. In the superposition (4.3), however, we can adjust the coefficients $a\left(l_{1}, l_{2}, p_{1}, p_{2}\right)$ such that the right hand side becomes again proportional to $\left|p_{1}, p_{2}\right\rangle$.

Observe that the action of the dilatation operator in the generic case is symmetric in $l_{1}$ and $l_{2}$. Hence, both the in-coming and the out-going waves acquire the same factor upon action with the dilatation operator originating from the exponentials. This factor is independent of the function $f$ since in the generic case the separation of the magnons is so large that only the leading 1 of the function $f$ of (4.7) contributes. It is also independent of the S-matrix which does not depend on the magnon positions at all. Therefore the generic equation determines the eigenvalue $q_{2}\left(p_{1}, p_{2}\right)$ alone. The eigenvalue splits into a sum

$$
q_{2}\left(p_{1}, p_{2}\right)=q_{2}\left(p_{1}\right)+q_{2}\left(p_{2}\right)
$$

\footnotetext{
${ }^{8}$ We use similar shorthands as before, e.g. $\left|\mathcal{O}_{l_{1} \pm m, l_{2}}\right\rangle:=\left|\mathcal{O}_{l_{1}-m, l_{2}}\right\rangle+\left|\mathcal{O}_{l_{1}+m, l_{2}}\right\rangle$.
} 
with

$$
q_{2}(p)=8 \sin ^{2}\left(\frac{p}{2}\right)-32 \Lambda_{r} \sin ^{4}\left(\frac{p}{2}\right)+256 \Lambda_{r}^{2} \sin ^{6}\left(\frac{p}{2}\right)-2560 \Lambda_{r}^{3} \sin ^{8}\left(\frac{p}{2}\right)+\ldots .
$$

This function is called "dispersion relation" or one-magnon energy. The boundary equations determine particular perturbative orders of $S$ and particular values of the $f_{i}$ 's. We summarize this in the following table:

\begin{tabular}{|c|c|c|c|c|}
\hline & $D_{2}$ & $D_{4}$ & $D_{6}$ & $D_{8}$ \\
\hline$l_{2} \geq l_{1}+5$ & \multirow{4}{*}{$\left.q_{2}(p)\right|_{\mathcal{O}\left(\Lambda_{r}^{0}\right)}$} & \multirow{3}{*}{$\left.q_{2}(p)\right|_{\mathcal{O}\left(\Lambda_{r}^{1}\right)}$} & \multirow{2}{*}{$\left.q_{2}(p)\right|_{\mathcal{O}\left(\Lambda_{r}^{2}\right)}$} & $\left.q_{2}(p)\right|_{\mathcal{O}\left(\Lambda_{r}^{3}\right)}$ \\
\hline$l_{2}=l_{1}+4$ & & & & $f_{0}\left(3, p_{1}, p_{2}\right)$ \\
\hline$l_{2}=l_{1}+3$ & & & $f_{0}\left(2, p_{1}, p_{2}\right)$ & $f_{1}\left(2, p_{1}, p_{2}\right)$ \\
\hline$l_{2}=l_{1}+2$ & & $f_{0}\left(1, p_{1}, p_{2}\right)$ & $f_{1}\left(1, p_{1}, p_{2}\right)$ & $f_{2}\left(1, p_{1}, p_{2}\right)$ \\
\hline$l_{2}=l_{1}+1$ & $S_{0}\left(p_{1}, p_{2}\right)$ & $S_{1}\left(p_{1}, p_{2}\right)$ & $S_{2}\left(p_{1}, p_{2}\right)$ & $S_{3}\left(p_{1}, p_{2}\right)$ \\
\hline
\end{tabular}

The form of $f$ is not very enlightening as it depends on the basis (expressed by the parameter $\alpha$ of $D_{8}$ ). The S-matrix is of course independent of $\alpha$ and we find

$$
S\left(p_{1}, p_{2}\right)=\frac{\varphi\left(p_{1}\right)-\varphi\left(p_{2}\right)+i}{\varphi\left(p_{1}\right)-\varphi\left(p_{2}\right)-i} e^{i \psi\left(p_{1}, p_{2}\right)}
$$

with the phase function

$$
\varphi(p)=\frac{1}{2} \cot \left(\frac{p}{2}\right)\left[1+8 \Lambda_{r} \sin ^{2}\left(\frac{p}{2}\right)-32 \Lambda_{r}^{2} \sin ^{4}\left(\frac{p}{2}\right)+256 \Lambda_{r}^{3} \sin ^{6}\left(\frac{p}{2}\right)+\ldots\right]
$$

and the exponent

$$
\psi\left(p_{1}, p_{2}\right)=104 \Lambda_{r}^{3}\left[\sin ^{2}\left(\frac{p_{1}}{2}\right) \sin p_{2} \sin ^{2}\left(\frac{p_{2}}{2}\right)-p_{1} \leftrightarrow p_{2}\right] .
$$

These formulas provide an eigenstate $\left|p_{1}, p_{2}\right\rangle$ with eigenvalue $q_{2}\left(p_{1}, p_{2}\right)$ for any value of $p_{1}$ and $p_{2}$. The discrete physical spectrum is specified not before imposing boundary conditions which lead to the Bethe equations and pick out a discrete set of Bethe momenta.

In the two-magnon case we impose the periodicity condition

$$
a\left(l_{1}, l_{2}, p_{1}, p_{2}\right)=a\left(l_{2}, l_{1}+L, p_{1}, p_{2}\right) .
$$

Now it pays off that we introduced the same function $f$ for in-coming and out-going wave, since this leads independent of $f$ to

$$
\exp \left(i L p_{1}\right)=S\left(p_{1}, p_{2}\right)=\exp \left(-i L p_{2}\right) .
$$

These are the two-magnon Bethe equations. Due to the originating trace structure the states of the spin-chain need to be invariant under cyclic permutations. This leads to the additional zero total momentum condition

$$
p_{1}+p_{2}=0 .
$$

The equations (4.16) and (4.17) completely determine the two-magnon spectrum, cf. Sec.4.4.

Finally let us remark that the energy shift of the four-loop "wrapping" state of Sec. 2.4 may also be brought into the apparently universal form (4.12) with the identical phase function (4.13) and the exponential factor $\psi_{\text {wrap }}(p,-p)=\frac{16034}{3} \Lambda_{r}^{3} \sin ^{4}\left(\frac{p}{2}\right) \sin p$ which only differs from (4.14) in the overall constant up front ${ }^{9}$.

\footnotetext{
${ }^{9}$ We thank S. Frolov for a discussion on this point.
} 


\subsection{Bethe equations}

The case of a spin-chain with $M$ magnons and total length $L$ is a straightforward generalization of (4.16). According to the notion of [22], in an integrable system a general scattering process is always factorized into two-body scattering sub-processes. Therefore one just needs to replace the right hand side of (4.16) by a product of S-matrices. Any magnon, represented by $p_{k}$, interacts once with every other magnon $p_{j \neq k}$ on its way around the spin-chain:

$$
\exp \left(i L p_{k}\right)=\prod_{\substack{j=1 \\ j \neq k}}^{M} S\left(p_{k}, p_{j}\right)=\prod_{\substack{j=1 \\ j \neq k}}^{M} \frac{\varphi\left(p_{k}\right)-\varphi\left(p_{j}\right)+i}{\varphi\left(p_{k}\right)-\varphi\left(p_{j}\right)-i} e^{i \psi\left(p_{k}, p_{j}\right)}
$$

for $k=1, \ldots, M$. This is supplemented by the zero total momentum condition

$$
\sum_{i=1}^{M} p_{i}=0
$$

As the derivation of (4.18) contained some subtle points we have checked the Bethe equation for a number of states. The results are documented in App. D

Let us now compare this form to the other known perturbative long-range Bethe ansätze in the literature. We note that the dispersion relation (4.11) and the phase function (4.13) are exactly the same as for the Beisert-Dippel-Staudacher spin-chain [8]. The difference lies in the presence of the exponential factor in the Bethe ansatz for the PWMT. This exponential is reminiscent of the Bethe ansatz for quantum strings proposed by Arutyunov, Frolov and Staudacher [14. There the exponent is a series of antisymmetric products of all conserved charges. This is exactly the same as we observe here, since our exponent is in fact

$$
\psi\left(p_{1}, p_{2}\right)=\frac{13 \Lambda_{r}^{3}}{8}\left[q_{2}\left(p_{1}\right) q_{3}\left(p_{2}\right)-q_{3}\left(p_{1}\right) q_{2}\left(p_{2}\right)\right]
$$

where

$$
q_{2}(p)=8 \sin ^{2}\left(\frac{p}{2}\right) \quad, \quad q_{3}(p)=8 \sin (p) \sin ^{2}\left(\frac{p}{2}\right)
$$

are the lowest order contributions to the eigenvalues of the higher charges $Q_{2}$ and $Q_{3}$, cf. (4.11) and (D.2). A more detailed comparison to the quantum string ansatz reveals that our exponent has one additional power of the coupling constant $\Lambda_{r}$. This explains why the exponential yields a breakdown of BMN scaling in our case, while it results in a sub-leading discrepancy in the near-BMN limit with respect to SYM theory in the quantum string case.

The existence of an exponential factor in the Bethe ansatz seems to be the generic form for integrable long-range spin-chains. It would be very interesting to determine whether the Bethe ansatz for the Inozemtsev spin-chain may be brought into this general form as well. The idea would be to trade contributions in the phase function for an exponential factor. 


\subsection{Two-magnon spectrum and violation of BMN scaling}

Using the above Bethe ansatz one may solve the two-magnon problem explicitly. Here, due to the zero total momentum condition $p_{1}=-p_{2}=: p$, one finds just one Bethe equation

$$
\exp (i L p)=\frac{\varphi(p)+i / 2}{\varphi(p)-i / 2} e^{i \psi(p,-p)} .
$$

This equation is solved via the perturbative ansatz

$$
p=p_{0}+\Lambda_{r} p_{1}+\Lambda_{r}^{2} p_{2}+\Lambda_{r}^{3} p_{3}+\ldots,
$$

plugging this into (4.22) and making use of (4.13) one finds the explicit result:

$$
\begin{aligned}
p_{0} & =\frac{2 n \pi}{L-1} \\
p_{1} & =-\frac{16 \cos \left(\frac{n \pi}{L-1}\right) \sin ^{3}\left(\frac{n \pi}{L-1}\right)}{L-1} \\
p_{2}= & \frac{64 \cos \left(\frac{n \pi}{L-1}\right) \sin ^{5}\left(\frac{n \pi}{L-1}\right)}{(L-1)^{2}}\left[2 L+(L+3) \cos \left(\frac{2 n \pi}{L-1}\right)\right] \\
p_{3}= & \frac{32 \cos \left(\frac{n \pi}{L-1}\right) \sin ^{5}\left(\frac{n \pi}{L-1}\right)}{3(L-1)^{3}}\left[\left(-79 L^{2}+122 L-79\right)+4\left(5 L^{2}-31 L+20\right) \cos \left(\frac{2 n \pi}{L-1}\right)\right. \\
& \left.\quad+4\left(4 L^{2}+13 L-20\right) \cos \left(\frac{4 n \pi}{L-1}\right)+4\left(L^{2}-7 L+10\right) \cos \left(\frac{6 n \pi}{L-1}\right)\right]
\end{aligned}
$$

Inserting these expressions into the (4.10) yields, upon expansion in $\Lambda_{r}$, the closed expression for the two-magnon spectrum $\delta \Delta_{r}=\Lambda_{r} q_{2}$ parametrized by the integers $n$ and $L$, where $L$ denotes the length of the spin-chain and $n=0, \ldots,\left[\frac{L-2}{2}\right]$ :

$$
\begin{aligned}
\delta \Delta_{r}(n, L)= & 16 \Lambda_{r} \sin ^{2}\left(\frac{n \pi}{L-1}\right) \\
& -\frac{64 \Lambda_{r}^{2}}{L-1} \sin ^{4}\left(\frac{n \pi}{L-1}\right)\left[(L+1)+2 \cos \left(\frac{2 n \pi}{L-1}\right)\right] \\
& +\frac{256 \Lambda_{r}^{3}}{(L-1)^{2}} \sin ^{6}\left(\frac{n \pi}{L-1}\right)\left[\left(2 L^{2}+5 L-2\right)+2(5 L+2) \cos \left(\frac{2 n \pi}{L-1}\right)+(L+4) \cos \left(\frac{4 n \pi}{L-1}\right)\right] \\
+ & \frac{256 \Lambda_{r}^{4}}{3(L-1)^{3}} \sin ^{6}\left(\frac{n \pi}{L-1}\right)\left[\left(-30 L^{3}-39 L^{2}+51 L-39\right)\right. \\
& +3\left(10 L^{3}-49 L^{2}+26 L-13\right) \cos \left(\frac{2 n \pi}{L-1}\right)+8\left(11 L^{2}-10 L\right) \cos \left(\frac{4 n \pi}{L-1}\right) \\
& \left.+\left(18 L^{2}+90 L-30\right) \cos \left(\frac{6 n \pi}{L-1}\right)+\left(2 L^{2}+17 L+30\right) \cos \left(\frac{8 n \pi}{L-1}\right)\right]
\end{aligned}
$$

Expanding this in the BMN limit, i.e. $\Lambda_{r}, L \rightarrow \infty$ with $\Lambda_{r} / L^{2}$ fixed, one finds

$$
\begin{aligned}
\lim _{\substack{L \rightarrow \infty \\
\Lambda_{r} \propto L^{2}}} \delta \Delta_{r}(n, L)= & 4\left(4 \pi^{2} n^{2} \frac{\Lambda_{r}}{L^{2}}\right)-4\left(4 \pi^{2} n^{2} \frac{\Lambda_{r}}{L^{2}}\right)^{2}+8\left(4 \pi^{2} n^{2} \frac{\Lambda_{r}}{L^{2}}\right)^{3} \\
& -6656 \pi^{6} n^{6} \frac{\Lambda_{r}^{4}}{L^{7}}\left(1-\frac{1}{L}\right)-20\left(4 \pi^{2} n^{2} \frac{\Lambda_{r}}{L^{2}}\right)^{4}+\ldots
\end{aligned}
$$


This is in fact divergent due to the four-loop term $\sim \Lambda_{r}^{4} / L^{7}$ : the proclaimed breakdown of BMN scaling. This formula is to be compared with the proper scaling behavior (3.7) with $(L \approx J)$. The additional (violating) piece originates from the microscopic contact interactions of two magnons.

\section{Conclusion and discussion}

In this paper we have performed a fourth order perturbative analysis of the $\mathrm{SU}(N)$ planewave matrix theory in its planar large $N$ limit in the closed minimal $\mathfrak{s u}(2)$ subsector. The outcome of our analysis yielded an effective planar Hamiltonian, which is integrable to this rather high loop order. Additionally we have explicitly constructed the first two higher commuting charges of the integrable system to the corresponding loop order, which is summarized in App. C We take these results as a very strong indication of the complete integrability of the planar matrix theory system and similarly so for the closely related "mother" theory of superconformal $\mathcal{N}=4$ Yang-Mills.

Moreover we determined the underlying perturbative asymptotic Bethe ansatz [22] of the system for states of length larger than four. While the form of the integrable spin-chain Hamiltonian (or planar dilatation operator or effective matrix model Hamiltonian) becomes more and more involved at higher loops, the "core" of the model - the two-magnon S-matrix $S\left(p_{1}, p_{2}\right)$ - is of the intriguingly compact form

$$
S\left(p_{1}, p_{2}\right)=\frac{\varphi\left(p_{1}\right)-\varphi\left(p_{2}\right)+i}{\varphi\left(p_{1}\right)-\varphi\left(p_{2}\right)-i} \times \exp \left(i f\left(\Lambda_{r}\right) \sum_{i=1}^{\infty} \Lambda_{r}^{i} q_{i+1}\left(p_{1}\right) q_{i+2}\left(p_{2}\right)-p_{1} \leftrightarrow p_{2}\right),
$$

which was first established for the quantum $A d S_{5} \times S^{5}$ string Bethe ansatz in [14. Here $q_{i}(p)$ denotes the higher charges of the spin-chain, $f\left(\Lambda_{r}\right)$ a function of the coupling constant and $\varphi(p)$ the phase relation of the model. This form of the S-matrix appears to be generic for integrable long-range spin-chains: Except for the Inozemtsev chain all known long-range spinchains or Bethe ansätze (the Beisert-Dippel-Staudacher chain [8], the Arutyunov-FrolovStaudacher quantum string chain [14, our plane-wave matrix theory effective Hamiltonian and the recently established S-matrices of the $\mathfrak{s l}(2)$ and $\mathfrak{s u}(1 \mid 1) \mathcal{N}=4$ dilatation operators 22] ) fall into the class (5.1) with the same functions $\varphi(p)$ and $q_{i}(p) !{ }^{10}$ It would be very interesting to establish whether the Inozemtsev chain S-matrix may also be brought into the form of Eq. (5.1).

Our perturbative computation was only attainable through the use of a tailor made computer program which generated all planar graphs and performed the necessary term algebra in a massively distributed fashion, which is reviewed in detail in App. A. The program should also be applicable for similar problems in planar perturbation theory at high orders, in particular for a similar analysis in larger closed subsectors of the plane-wave matrix model, such as $\mathfrak{s u}(2 \mid 3)$.

\footnotetext{
${ }^{10}$ Strictly speaking this is not quite correct as the fermionic $\mathfrak{s u}(1 \mid 1)$ chain has a trivial phase relation $\varphi(p)=0[22$.
} 
The motivation for this computation grew out of the close relationship of the effective plane-wave matrix model Hamiltonian to the dilatation operator of $\mathcal{N}=4$ super YangMills, which is firm in the joint minimal $\mathfrak{s u}(2)$ subsector up to the three loop order [21, 6]. This connection stems from the consistent (classical) reduction of the full field theory on a three-sphere [2] and is seemingly stable under quantum corrections (up to three-loops) resulting in a "renormalization" of the relation between the matrix model (dimensionless) mass parameter $M$ and the super Yang-Mills coupling constant $g_{\mathrm{YM}}$

$$
\Lambda=\Lambda_{r}+\frac{7}{2} \Lambda_{r}^{2}-11 \Lambda_{r}^{3}+\frac{1257}{16} \Lambda_{r}^{4}+\mathcal{O}\left(\Lambda_{r}^{5}\right)
$$

where $\Lambda=2 N / M^{3}$ and $\Lambda_{r}=g_{\mathrm{YM}}^{2} N / 4 \pi$. Here the highest order term was determined by requiring "naive" BMN scaling, i.e. demanding that the four-loop contribution to the effective Hamiltonian acts as a clean lattice laplacian to the fourth power on well separated magnons. It remains to be shown whether the established correspondence of plane-wave matrix theory to the $\mathcal{N}=4$ dilation operator of the full uncompactified field theory extends beyond the three-loop level (in the planar sector). For this the four-loop anomalous scaling dimensions of one or two super Yang-Mills operators would need to be computed. Hence unfortunately our verdict on the perturbative BMN scaling of the full $\mathcal{N}=4$ gauge theory at four loops cannot be conclusive, it, however, does certainly question its existence beyond three-loops. A better understanding of the inner workings of the planar plane-wave matrix theory $/ \mathcal{N}=4$ super Yang-Mills correspondence on the basis of an effective field theory approach in which one integrates out the higher Kaluza-Klein modes of the 4 d gauge theory, would be of great importance.

Our results also shed light on the structure of the "wrapping" interactions for long-range spin-chains, which are inaccessible through the means of the asymptotic Bethe ansatz where the length of the chain needs to be strictly larger than the spread of the local Hamiltonian: In the considered $\mathfrak{s u}(2)$ subsector this happens for the first time at four-loop order, where the resulting spin-chain Hamiltonian involves five neighboring spin interaction terms, whereas the shortest non-protected operator in this subsector is a Konishi descendant of length four: $\operatorname{tr}\left[Z^{\dagger}, W^{\dagger}\right]^{2}$. Its four-loop energy shift can neither be accounted for by any "natural" prescription of how to act with the five spin terms of the Hamiltonian on a length four state nor by blindly applying the Bethe ansatz. One finds

$$
\delta \Delta_{r}=12 \Lambda_{r}-48 \Lambda_{r}^{2}+336 \Lambda_{r}^{3}- \begin{cases}2958 \Lambda_{r}^{4} & \text { (wrap around) } \\ 3026 \Lambda_{r}^{4} & \text { (discard long-range terms) } \\ 3054 \Lambda_{r}^{4} & \text { (Bethe ansatz) } \\ \frac{12771}{4} \Lambda_{r}^{4} & \text { (true value) }\end{cases}
$$

More work is needed to understand how this intrinsic restriction of the asymptotic Bethe ansatz could be overcome.

Finally it would be very nice to establish a proof of the integrability of the planar plane-wave matrix theory. Compared to the situation for the $\mathcal{N}=4$ super Yang-Mills field theory this appears to be more tractable, as one has access to the full non-perturbative 
Hamiltonian. However, efficient techniques to isolate the planar large $N$ sector in a nonperturbative fashion are lacking.

\section{Acknowledgments}

We would like to thank Gleb Arutyunov, Niklas Beisert, Virginia Dippel, Sergey Frolov and Matthias Staudacher for crucial discussions and comments.

Moreover we are greatly indebted to the members of the computational division of the GEO 600 gravitational wave detector project, especially Maria Alessandra Papa and Steffen Grunewald, for allowing us to use their Merlin cluster for part of our calculations and to the experimental particle physics group at the University of Wuppertal, especially Hendrik Hoeth, for computation time on the presently largest German university computer the ALiCEnext Linux cluster. Minor parts of the calculation have been performed at the cip.physik.uni-muenchen.de computer lab of the University of Munich, so we want to thank both the manager as well as the head administrator of that lab - Sigmund Stintzing and Susanna Maurer. Other parts have been done on workstation machines of the AEI, hence we also want to thank the AEI's administrative staff, especially the head Christa Hausmann-Jamin, for their support. We also want to thank Peter Herzog for allowing us to use the spotlight.de webserver for our purposes which played a key role in coordinating the distribution of parts of the calculation to individual machines via HTTP. Furthermore, the authors thank Klaus Aehlig for his permission to include his very beautiful solution to a functional programming exercise problem that plays a minor role in our approach into our source code. Thomas Fischbacher acknowledges financial support by the German Academic Exchange Service via the DAAD postdoc research program. This work was partially supported by IISN - Belgium (convention 4.4505.86), by the "Interuniversity Attraction Poles Programme - Belgian Science Policy" and by the European Commission FP6 programme MRTN-CT-2004-005104, in which Thomas Fischbacher is associated to V.U. Brussel. 


\section{A Details on the Computation}

The problem solved by the gemstone code (which was developed for this application) is the generation of planar multiloop graphs from large sets of vertices with directed and colored legs, which have a term algebra associated to them, with a highly efficient algorithm that furthermore is implemented close to the machine language level for maximum speed.

Considering the technical complexity of the calculation of the dilaton operator at four loops, the authors have strong reason to believe that this may well be the largest symbolic calculation performed so far ${ }^{11}$.

The key to its successful completion was the development of aggressively optimized symbolic algebra code. With this program, which the authors named gemstone ${ }^{12}$, the three-loop calculations that have been done in 21] (by using the already highly optimized FORM [26] symbolic manipulation program and a more conventional algorithm) could be checked in about $1 / 100$ of the original calculation time.

As this code is at present the best tool available to systematically do many more planar higher-loop matrix model calculations, and due to its modularity should be easily adoptable to planar high-loop field theory calculations as well, it might be quite interesting in itself and has been included in the source archive of the arXiv.org preprint of this work at http://www . arxiv.org/e-print/hep-th/0412331

Furthermore, as the results presented in this work hinge on the correctness of the algorithm as well as its implementation, the scientific demand of verifiability gives another strong incentive to make this code publicly available.

Finally, many of the approaches to the problem of making the calculation fast are generic enough to be useful in many other situations, even if not of direct relevance to the physics of this problem. As this appendix may be interesting to computer scientists as well, it is essentially self-contained.

\section{A.1 The Task}

In a nutshell, the problem at hand consists of the determination of perturbative quantum corrections to the effective Hamiltonian that come from interaction diagrams which can be drawn without self-intersections (i.e. are planar). Conceptually, it may be obtained by a simple algebraic normal ordering procedure with subsequent isolation of those contributions that can be associated to planar graphs. While this approach is very simple, it is quite limited in practice by excessive growth of the number of terms when increasing the perturbative order. As both quantitatively and qualitatively new phenomena appear when going to higher orders, doing such calculations is not merely an academic exercise. Hence, one should make use of as much of the specific structure of the problem as possible in order to reduce the complexity of high-order calculations. In our case, this especially means to make as much use of the planarity property as possible right from the beginning, instead of generating all terms and later throwing away contributions that can be identified as coming

\footnotetext{
${ }^{11}$ There are far larger massively distributed prime searches as well as encryption breaking attempts, but the underlying questions should perhaps not be regarded as being of symbolic nature.

${ }^{12}$ as compactness of code and flawlessness are the most important virtues for such a task
} 
from nonplanar graphs. While the number of nonplanar graphs also increases dramatically with increasing number of vertices, there are nevertheless far less planar than non-planar graphs in all problems of interest.

We have to find all graphs with certain pre-defined properties, associate a term to every graph, and sum all the contributions from allowed graphs. Graphs are built from vertices, which may have up to four legs, by successively adding vertices and fusing legs. Every vertex leg has a direction (in-going or out-going, corresponding to particle creation or annihilation) as well as a color (corresponding to particle type). Only matching legs (same color, different directions) may be fused. To every vertex, we associate an algebraic factor depending on the type of the vertex. Leg fusion induces algebraic transformations.

Vertices have three or four legs, except for some degenerate cases where self-binding of a four-leg vertex gives a two-leg vertex. The factors associated to three-leg vertices contain a single power of the interaction strength $g$, while those associated to (possibly degenerate) four-leg vertices contain a factor $g^{2}$. These two large vertex classes are further subdivided by considering the energy shift associated to particular vertices - this is just the mass of the particles created (represented by out-going legs) minus the mass of the particles destroyed, and can be represented by a small integer. With this additional distinction, there are 15 different vertex classes, each containing between 1 and 188 different types of vertices.

In a first step, we expand the Hamiltonian into a set of sequences of vertex classes. As this is of minor relevance for the heart of the calculation, this step is discussed separately in section A.4. For the fourth-order calculation, this yields 338834 individual sequences, each carrying an interaction strength factor $g^{8}$.

Next, an initial closed chain of vertices with all legs on the outside has to be chosen. Starting from this chain, we have to consider every single sequence of vertex classes individually, finding all possible realizations of that sequence as a sequence of vertex types. These building blocks then have to be fused subsequently to the initial chain in all possible ways, subject to some extra constraints. The fundamental graph operations, such as fusing legs, induce transformations on the associated terms that have to be kept track of. Eventually, all terms from admissible final graphs have to be summed.

As an example, the vertex class sequence consisting of four times the largest class with 188 four-leg vertex types in it alone gives rise to $188^{4}=1249198336$ individual sequences of vertex types. For every single one of those, all possible ways to add these vertices in sequence to the starting graph have to be taken into consideration. Likewise for the other 338833 sequences. This is depicted schematically in figure 1.

For the determination of the four-loop dilatation operator, it suffices to form all additions of all these sequences of vertices to a suitably chosen initial chain of length eleven.

Vertices to be added may be turned, but not flipped over. Every final graph must obey the following constraints:

1. It is planar.

2. All the open legs are on the outside.

3. Its construction by successive addition of vertices never involved a step where an in-going leg remained open (as this would act on the vacuum and give zero). 


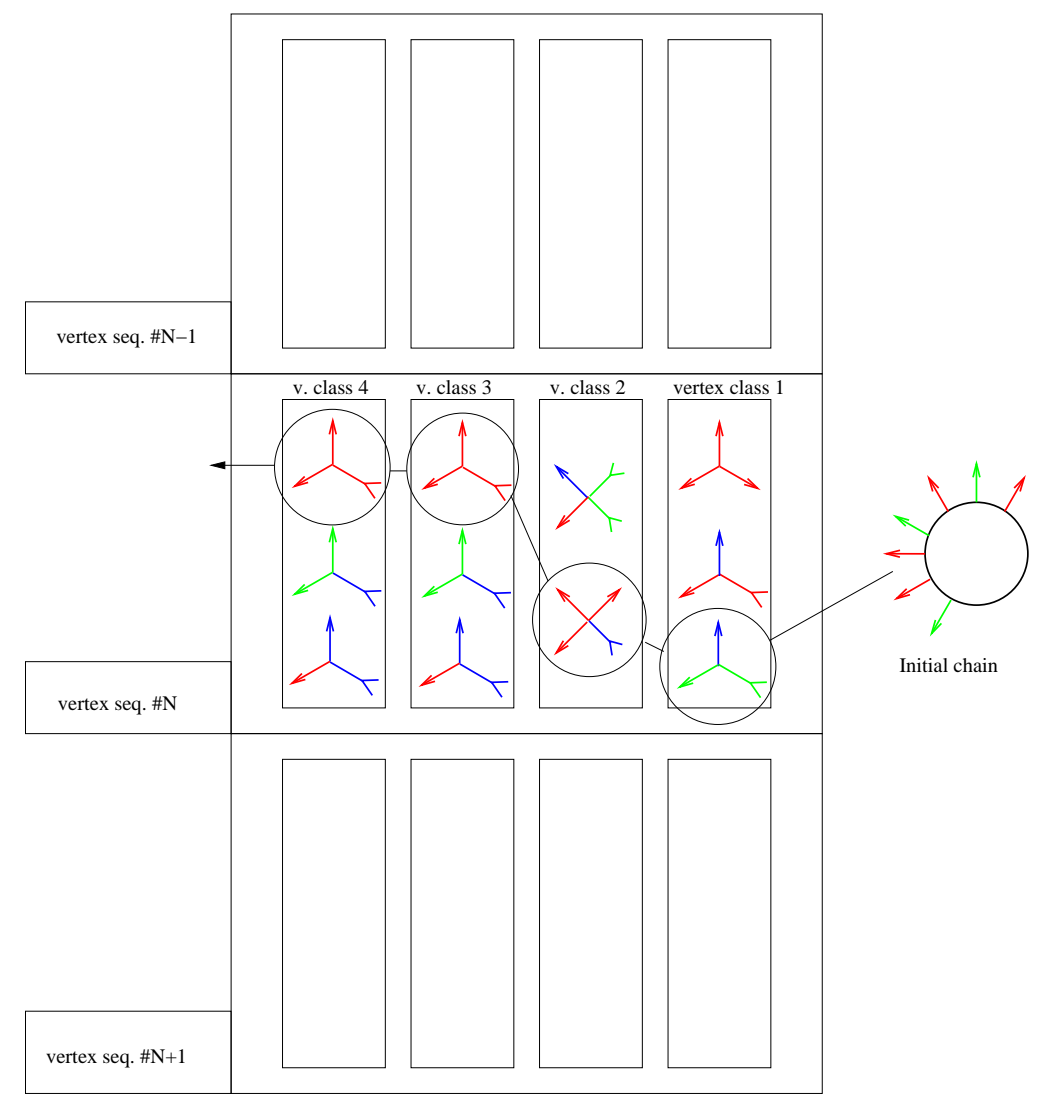

Figure 1: Forming all Graphs: One particular choice where the last two vertices to be added are of the same type. 
As an extra difficulty, some legs are fermionic, which means that care of extra factors -1 has to be taken which occur in some (but not all) situations when such legs are fused.

\section{A.2 Choice of the Platform}

As a basis for the implementation, the Objective CaML 30] system has been chosen (an early prototype was written in LISP). This decision was based on the expectation that the four-loop calculation might become almost prohibitively large, and hence one should be able to make use of computer power donated by volunteers to run the calculation in a highly parallelized scheme, similar to efforts like SETI@Home 31, GIMPS [32, and many others. This requires the availability of a high-quality native code compiler for a reasonable price (ideally for free) for all intended target platforms, especially including Linux/x86, Microsoft Windows, Mac OS X, and possibly even Linux on non-PC hardware. This excludes LISP as an implementation language, since there is no free LISP Compiler of sufficient strength available for the Windows platform. Java as well as $\mathrm{C}$ (or $\mathrm{C}++$ ) are not considered viable options as well, as some of the algorithmic tricks that had to be used are prohibitively clumsy to express in such languages that do not provide sufficient support especially for lambda abstraction; furthermore, code development and in particular debugging is facilitated greatly by the availability of an interactive command prompt where one can immediately test program components individually. This is also not widely available for the well-known mainstream languages.

The decision to use Objective CaML was further supported by Ocaml's excellent record for being a versatile tool to quickly build solutions for challenging problems [29], availability of key algorithmic libraries (hashes, rational numbers, etc.), as well as quite positive experience with this system in an earlier research project from the side of one of the authors.

\section{A.3 Planar Graph Generation}

As explained, we are interested in planar graphs only, as this restriction will considerably constrain the number of graphs.

In terms of data structures, one attractive way to organize this calculation is to represent every graph as a vector of connected components, where every connected component is a vector of domains (cells) that carry legs along their boundaries and are glued together in such a way that all legs point to the inside. To every connected component, we associate a particular term - details of the term algebra will be discussed in the next section.

The cells may themselves be represented as vectors of legs, and every leg has to know about its type (direction and color) and furthermore some kind of pointer (in a general, not implementation-specific sense) into the term of the leg's connected component. In the implementation, all this information is recorded in a single signed 30-bit integer per leg. We make use of the observation that for a four-loop calculation, we will introduce at most $8 \cdot 3=24$ legs from vertices in addition to the 11 legs in the initial sequence. This means that we can conveniently label legs by 6 -bit numbers in the range $0 \ldots 35$. (Additional legs may be introduced by tracing fermionic loops of odd length - this gives an extra factor $\epsilon^{i j k}$ 

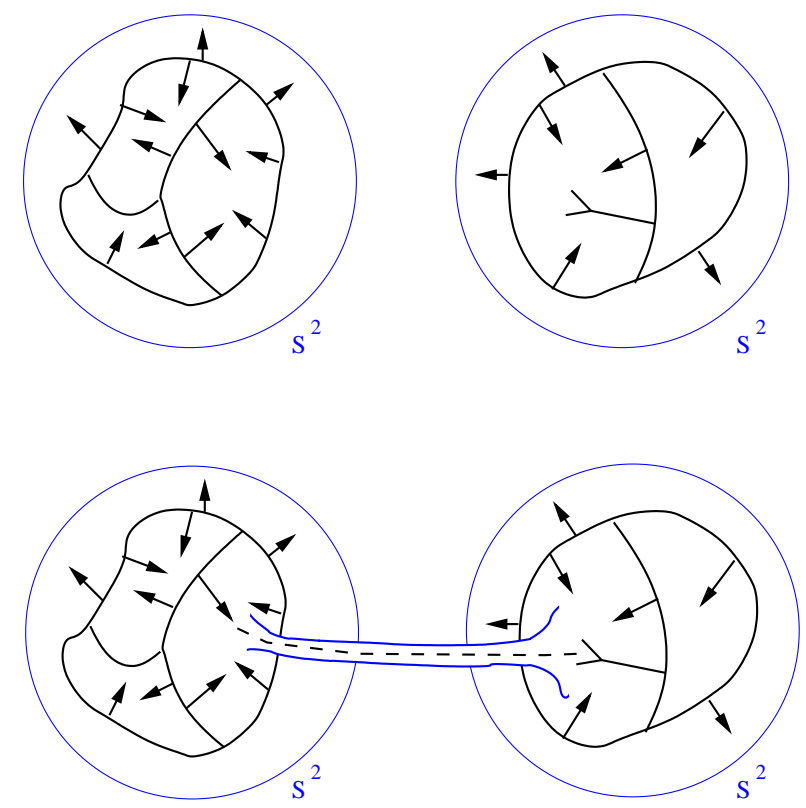

Figure 2: Fusing legs

from the projector. There may be at most two such factors in our calculations, so we take labels for these indices from the upper end of the 6-bit integer range.)

Fundamental operations on graphs are (1) the addition of a new vertex, which initially is regarded as an individual connected component with only one cell, (2) the composition of two connected components right before legs between them are fused: this causes one entry to be removed from the vector of all connected components and another one to be enlarged, as well as corresponding transformation on the vector of associated terms, where one entry is removed and multiplied into another one, and (3) connection of cells by fusion of legs. This reduces the number of cells in a connected component and as well induces certain transformations on the associated term.

Concerning the fusion of legs, it is helpful to think of each individual connected components as living on a sphere. When legs are fused, these spheres are punctured at the interior of the partaking cells and then connected appropriately, see figure 2.

Note that steps (1) and (3) introduce a choice among many different possibilities. In (1) this comes from (in some cases, quite many) different vertex types contributing to a vertex class, all of them having individual leg structure, hence requiring to branch graph generation, in (3) this comes from the possibility to connect the legs from two domains in multiple different ways. In the implementation, the technique of continuation coding turned out to be a very convenient way to express the branchings in these steps of the calculation. That is, one passes to the function $b$ that branches the calculation a function $f$ which is to be called with an individual branching and does all the remaining processing for this branch. ("Passing around the entire rest of the calculation as a function argument.") 
This is then called multiple times, once for every branch. The abstract idea of passing around code towards the data that have to be processed - like an inverse assembly line that transports workers to partially completed pieces - is a re-occurring pattern in the design of the entire system. While it is nice and highly expressive to write the code in such a way, dynamic memory allocation (aka 'consing') of functions with partially fixed parameters (aka 'closures') is a bit wasteful in terms of efficiency. With a lot more work, some (limited) additional efficiency improvement might be gained here.

A further subtlety comes from the requirement to properly treat signs from fermionic loops. This is done by subsequently recording the types of all vertex's legs (fermionic/nonfermionic) in a special bit-vector in the graph (in the implementation, this is distributed out over two 30-bit integers) whenever a new vertex is added, and removing the fermionic leg bits again whenever two such legs are being fused, introducing an extra minus sign if there is an odd number of active fermionic legs whose label lies between the labels of the fused legs. (Care must be taken to ensure that one does not apply such modifications to the graph data structure in such a way that they are not being undone properly once we go back to do a different branch of the calculation.)

A final trick is to make use of the property that in every vertex addition step, the only legs that may be fused are those that were introduced by the vertex. It may well be that this eventually leads to the combination of (at worst) five different connected components (the vertex itself and four other components that bind to its four in-going legs), but this can be done sequentially, and in every such absorption step, we only have to remember the presently 'hot' connected component plus the cell where the vertex originally was absorbed, and the positions of the vertex's original still unbound legs in the new cell. As every leg can be labeled with a 6 -bit integer, and as we at most have four active ('hot') legs at any step during binding, it becomes attractive to encode a list of four legs in one 28-bit integer, seven bits per leg, with $127=1111111_{2}$ as a special 'end of list' stop code. Implementing primitive list operations on such short lists of small integers brings the benefit that no expensive memory management is required, plus a lot of work can be done very close to the processor's register file. Furthermore, should microprocessor design evolve into the highly desirable direction to have certain parts of freely reconfigurable logic on the CPU (cf. e.g. 27]) during the next decade, this specific part of the calculation could easily be moved directly to the hardware level, so that it could be done at virtually no cost at all.

The term algebra is fully abstract, which means that one may hook arbitrary implementations that provide notions of fusing terms and legs into the combinatorial engine. In particular, two term algebras that are particularly nice for debugging are the trivial one that just records all fusions in a list, and one that produces a graphviz [28] input file for visual rendering of individual graphs to check that the algorithm is working correctly.

\section{A.4 Operator Sequences}

The set of all vertex sequences that have to be applied to the initial chain is determined by expanding the effective Hamiltonian (B.1) in terms of vertices of definite energy shift. This allows one to shift all projectors and propagators out of the Hamilton operator. The corre- 
sponding rules are ( $V_{1}$ denoting three-legged vertices and $V_{2}$ denoting four-legged vertices):

$$
\begin{array}{ll}
V_{1} & \rightarrow \sum_{k \in\{ \pm 1, \pm 2, \pm 3\}} V_{1, k} \\
V_{2} & \rightarrow \sum_{k \in\{-4 \ldots 4\}} V_{2, k} \\
P(E) V_{\{1,2\} m} & \rightarrow V_{\{1,2\} m} P(E-m) \\
\Delta(E) V_{\{1,2\} m} & \rightarrow V_{\{1,2\} m} \Delta(E-m) \\
P(E)|\psi\rangle & \rightarrow \delta_{E, 0}|\psi\rangle \\
\Delta(0)|\psi\rangle & \rightarrow 0 \\
\Delta(E)|\psi\rangle & \rightarrow 1 / E|\psi\rangle \quad(E \neq 0)
\end{array}
$$

Thus, the one-loop operator (compare (B.5))

$$
T_{2}=P V_{2}+P V_{1} \Delta V_{1}
$$

is unfolded to a sum of seven terms:

$$
\begin{aligned}
T_{2}= & -\frac{1}{3} V_{1,+3} V_{1,-3} \\
& -\frac{1}{2} V_{1,+2} V_{1,-2} \\
& -V_{1,+1} V_{1,-1} \\
& +\frac{1}{3} V_{1,-3} V_{1,+3} \\
& +\frac{1}{2} V_{1,-2} V_{1,+2} \\
& +V_{1,-1} V_{1,+1} \\
& +V_{2,0} .
\end{aligned}
$$

At four-loop order, the corresponding operator contains 339 summands, which likewise unfold to 338834 contributions (compare (B.8)):

$$
\begin{aligned}
T_{8}= & \frac{5}{2187} V_{1,+3} V_{1,-3} V_{1,+3} V_{1,-3} V_{1,+3} V_{1,-3} V_{1,+3} V_{1,-3} \\
& +\frac{29}{3888} V_{1,+3} V_{1,-3} V_{1,+3} V_{1,-3} V_{1,+3} V_{1,-3} V_{1,+2} V_{1,-2} \\
& +\frac{17}{243} V_{1,+3} V_{1,-3} V_{1,+3} V_{1,-3} V_{1,+3} V_{1,-3} V_{1,+1} V_{1,-1} \\
& +338830 \text { more } \\
+ & \frac{1}{128} V_{2,-4} V_{2,-4} V_{2,+4} V_{2,+4}
\end{aligned}
$$

Every such factor $V_{\text {, }}$. may again be a sum of up to 188 contributions, each one with its own leg structure.

While this is sufficiently easy to do with a small FORM program within about 15 minutes of calculation time on $1.7 \mathrm{GHz}$ Pentium-IV hardware, we note that just as well here, it is possible with very little effort to speed up this part of the calculation by a factor of about 300 by using compiled (e.g. Lisp) code. As propagators and projectors are shifted through the term, it makes sense to just record and operate on the positions of all these factors that still are present instead of generating new terms even for shifts that only introduce an extra factor. One key observation is that there are only 15 different types of vertices, so one can encode a sequence of up to eight vertices very conveniently in a 32-bit number. (As the authors originally hoped to be able to go even beyond level four, and as the Lisp system with which they performed this expansion does not support 64-bit numbers, they abused 52-bit floatingpoint mantissas for this purpose.) These tags for vertex sequences are used as hash keys to collect contributions to the various sequences. 


\section{A.5 Term algebra}

Playing around with the graph engine shows that, even for small problems, quite impressive amounts of data have to be processed by the term algebra. As required by the trivial observation known as Amdahl's law that it helps little for speeding up a program by orders of magnitude to make just part of the code much faster that originally consumed half of the calculation time, an aggressive implementation of graph combinatorics must be matched by an equally aggressive term algebra.

\section{A.5.1 Rules}

All the terms that occur in the calculation are sums of contributions that consist of a rational coefficient, optionally with an extra factor $i$ or $\sqrt{2}$, and a series of further factors from the $\operatorname{spin}(9)$ algebra of types $\delta_{i j}, \delta_{a b}, \epsilon_{i j k}, \epsilon_{m_{1} \ldots m_{9}}, \gamma_{\alpha \beta}^{i}, \gamma_{\alpha \beta}^{a}, \Pi_{\alpha \beta}^{ \pm}$. Here, $i, j, \ldots$ denote $\mathrm{SO}(3)$ vector indices, $a, b, \ldots$ denote $\mathrm{SO}(6)$ vector indices and $m, \alpha$ are $\operatorname{spin}(9)$ vector and spinor indices for $\mathrm{SO}(3) \times \mathrm{SO}(6) \subset \operatorname{spin}(9)$. A projection factor $\Pi^{ \pm}=\frac{1}{2}\left(1 \pm \frac{1}{6} i \gamma^{i j k} \epsilon_{i j k}\right)$ has to be inserted wherever two fermionic legs are fused - cf. (2.3):

$$
c_{\alpha} c_{\beta}^{\dagger} \rightarrow \frac{1}{2} \Pi_{\alpha \beta}^{-} .
$$

These projection matrices are shifted through a sequence of $\gamma$ matrices via the rule $\gamma^{i} \Pi^{ \pm}=$ $\Pi^{ \pm} \gamma^{i}, \gamma^{a} \Pi^{ \pm}=\Pi^{\mp} \gamma^{a}$. Note that $\left(\Pi^{ \pm}\right)^{2}=\Pi^{ \pm}$and $\Pi^{+} \Pi^{-}=0$, as advertised for projectors.

As we work with a $\mathrm{SU}(2)$ subgroup sector of $\mathrm{SU}(4)=\operatorname{Spin}(6)$, we further split the $a$ indices via $a \rightarrow(W, \bar{W}, Y, \bar{Y}, Z, \bar{Z})$; we will then be only concerned with initial graphs formed from out-going $W, Z$ legs.

Explicitly, our $\operatorname{spin}(9)$ algebra reduction rules are:

- (delta) $\delta_{?_{1} ?_{2}} T_{\ldots ?_{1} \ldots}=T_{\ldots ?_{2} \ldots}, \delta_{a a}=6, \delta_{i i}=3, \delta_{\alpha \alpha}=16, \delta_{X \bar{X}}=\delta_{\bar{X} X}=\delta_{Y \bar{Y}}=\ldots=1$

- (epsilon) $\epsilon_{i j k} \epsilon_{i l m}=\delta_{j l} \delta_{k m}-\delta_{j m} \delta_{k l}$

- (Clifford) $\gamma_{\alpha \beta}^{i} \gamma_{\beta \gamma}^{j}+\gamma_{\alpha \beta}^{j} \gamma_{\beta \gamma}^{i}=2 \delta_{i j} \delta_{\alpha \gamma}$

- (epsilon-9) $\delta_{\alpha_{1} \alpha_{1} 0} \prod_{k=1}^{9} \gamma_{\left.\alpha_{k} \alpha_{(} k+1\right)}^{i_{k}}=16 \epsilon_{i_{1} \ldots i_{9}}^{(9)}, \quad \delta_{i j} \epsilon_{\ldots i \ldots j \ldots}^{(9)}=0$

There would be further (obvious) reduction rules involving e.g. $\epsilon^{(9)} \cdot \epsilon^{(9)}$, but we do not have to take care of those, as they cannot occur up to four-loop order. Likewise, we do not include rules of the type $\epsilon^{(9)} \epsilon^{(3)} \rightarrow \epsilon^{(6)}$ - the number of such terms is expected to be quite limited, and treatment of such special cases may always be deferred to more generic term manipulation programs.

The Clifford and epsilon-9 rules are not used in the form given here, but to determine beforehand a table of all the expansions of traces of gamma matrices in terms of epsilons and deltas that can show up in the calculation. 


\section{A.5.2 Strategy}

Aside from some special cases $\left(\epsilon \epsilon \rightarrow \sum \delta \delta\right.$ and $\left.\operatorname{tr} \gamma \ldots \gamma \rightarrow \sum \ldots\right)$, every graph contributes to the coefficient of a single individual summand. Hence, we associate to every connected component a tight encoding of a summand which contains information about the coefficient, certain extra factors like $i$ or $\sqrt{2}$, as well as $\epsilon_{i j k}, \epsilon_{m_{1} \ldots m_{9}}, \delta_{m n}$ and $\gamma_{\alpha \beta}^{m}$ algebraic factors and indices. Once a contribution has been generated, the new coefficient is added to a hash table mapping normalized summands to (references to) coefficients.

Using conventional symbolic algebra, fusing legs would be performed by generating a new summand which is just the old one extended by an extra $\delta_{m n}$ (or $\Pi_{\alpha \beta}^{ \pm}$) factor and then successively searching through all algebra reduction rules to find a place in the new term where some such rule can be applied. One can do much better by making use of the information that the original terms have been totally reduced and therefore, if any new reduction became possible, it has to include the newly added factor. Hence, we mark the newly added factor as 'hot' and successively chase it through all possible reduction rules that such a hot factor may participate in. Hotness is treated as contagious here: every factor that is 'activated' by a hot factor becomes hot itself.

In our particular case, such an approach stratifies the algebra in the sense that the longest chain of reductions that a newly introduced factor may generate is a hot gamma closing a trace which then is expanded to a set of hot deltas, which connect different epsilons that originally were connected to the gamma chain to produce another set of hot deltas, which may rename open legs (on other $\delta, \epsilon$, or $\gamma$ factors). No longer sequence of reductions than this $\gamma \rightarrow \delta \rightarrow \epsilon \rightarrow \delta$ chain may occur; in particular, there are no loops in our reduction rules that may be performed an unspecified number of times. Thus, at the heart of the term algebra lies a set of functions that represent this chain of reductions which may be entered at an arbitrary place, depending on the type of factor that has been added. As allocating memory for the many intermediate terms that have to be generated while a summand runs through this reduction chain would cause an excessive number of expensive memory reclaiming steps (garbage collection), we reduce dynamic memory allocation to a minimum by first copying summands that have to be reduced to a statically allocated space of sufficient size, which we call the 'workbench'; term transformations are directly applied to a term's copy on the workbench. The problem that one reduction step may branch into many different individual summands is then also easily dealt with: instead of a single workbench area, we use multiple such areas, start by copying the summand to area $\# 0$, and whenever the calculation branches into multiple summands, e.g. via $\epsilon_{i j k} \epsilon_{i m n} \rightarrow \delta_{j m} \delta_{k n}-\delta j n \delta_{k m}$, we copy the term that is currently processed in area $\# N$ to area $\#(N+1)$ multiple times, once for every branch, add appropriate modifications to it, and continue processing with the terms in place $\#(N+1)$, one after the other. Eventually, when no more reductions can be performed, we create a new summand out of the contents of the workbench area we presently work at.

This is depicted in diagram 3. (Admittedly, this description is slightly simplified.) The method employed here to chase active parts through a term in order to avoid both unnecessary checking whether some reduction rule can be applied as well as dynamical memory management is quite general and can be applied in every situation where reductions have to 


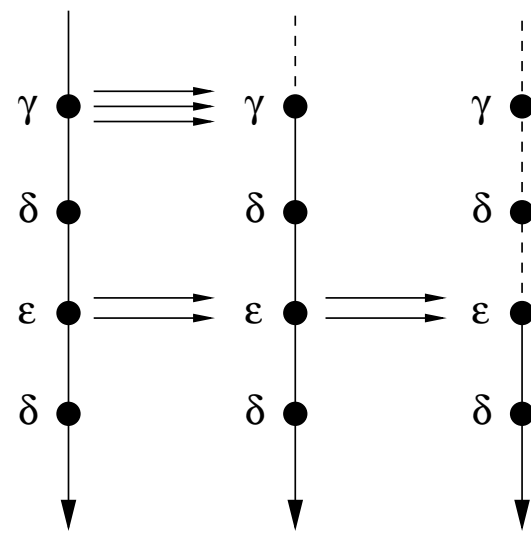

Area $0 \quad$ Area $1 \quad$ Area 2

Figure 3: Calculations on terms without dynamic memory management

be performed on terms of which one knows that they are derived from non-reducible terms by a small number of local modifications. Indeed, it should even be possible to implement a system that automatically compiles term reduction rules to machine code that employs such a 'waterfall' scheme.

The drawback of the decision to branch graph generation to individual summands obtained in $\epsilon \epsilon$ and $\operatorname{tr} \gamma$ reductions is that some graph generation work is re-done spuriously that could be avoided if one attributed sums, not products, to individual graphs. On the other hand, this scheme helps cutting some branches in the graph generation (to be discussed later). One should notice, however, that a large number of vertices has to be used to construct a graph that branches into many summands; as there are only at most eight vertices in total, the amount of work that is unnecessarily multiplied is limited to the tips of the graph of all possible choices. The most important advantage if this scheme is, of course, that it is comparatively easy to implement and debug.

As a further trick, one notes that besides the coefficients of individual vertex sequences, and symmetrization factors $1 / 6$ that come from $\Pi^{ \pm}$, all the denominators that appear in the calculation are small powers of two, with the numerators not becoming excessively large. One can convince oneself that up to four loops, floatingpoint calculations are exact on such numbers(!), so we can abuse double precision floatingpoint numbers to represent coefficients for a large part of the calculation and handle the other fractional factors not of this form in a separate final step. Besides vastly simplified memory management, this especially speeds up multiplication of coefficients.

\section{A.5.3 On the Graph $\leftrightarrow$ Term interplay}

Planar graph generation drives the calculation. Naively, one may consider to just memorize all the leg fusions during graph generation and eventually do all of the calculation of the term corresponding to a graph in the very last step. Alternatively, one may do as much of 
the calculation as possible in every step throughout the incremental generation of a graph. The advantage of the first approach is that no term calculations at all have to be performed for such intermediary graphs that cannot lead to a final graph of the desired topology. The big disadvantage is that for different final graphs that are derived from some common stem, the part of the calculation that corresponds to this stem is done multiple times, once for each final graph, although in principle it would have been sufficient to do it only once.

The big advantage of the second approach is that we may be able to see early that some stem cannot give any contribution anymore, e.g. if the coefficient became zero due to occurrence of a factor $\epsilon_{i i j}$ or a $\Pi^{+} \Pi^{-}$projector combination, and we may save a lot of expensive graph generation work for all graphs that are derived from this stem. Unfortunately, we then have to do lots of spurious term calculations for stems which will not produce a suitable final graph.

Interestingly, it is possible to get the best of both approaches by using so-called lazy evaluation (see figure 4): instead of triggering calculations on actual terms, we let the graph engine generate promises to do such calculations if needed. Such a 'promise' is basically a data structure holding a flag whether the promise was fulfilled already, plus either the value of this promise (if the flag says it indeed already was fulfilled), or otherwise a function, plus arguments, that, when evaluated, will produce that value. Once such a promise is requested to be fulfilled ('forced'), the promise's flag will be set accordingly, and the function and arguments will be replaced by the promise's value, so that next time its value is needed, one can just take it, without having to call the function again. Promises are comparatively cheap to give - this only requires a small amount of consing. Conceptually, the idea behind such promises is quite similar to the idea behind stock options. In practical applications, a 'promise' will typically promise to first require some other promises it depends on to be fulfilled, and use their values to compute its own value.

Here, we only carry promises for terms which depend on other promises for terms through the depth-first graph generation until we actually do generate a final graph. No expensive term calculations have been done so far, especially not for futile partial graphs that cannot be completed to a topologically interesting graph. Then, we take the graph's promise for a term and force it. It may be that this is zero, due to some promise in the stem producing a zero result, and we did some small amount of unnecessary work in generating this graph. However, when depth-first graph generation proceeds to the other branches that share a stem whose term is zero with the present graph's genealogy, we see that the promise for the term in the stem already has been fulfilled and we can cut generating further graphs from this particular stem.

One subtlety lies in the treatment of fermionic signs: the determination of signs from fusion of fermionic legs has to be taken out of the promise, as one has little control over the point in time when a promise is being fulfilled, and the state of the flags denoting which legs are unbound fermionic ones may be a very different one from the state of those flags at the time when the promise was given (which is the proper state to base sign decisions on). 


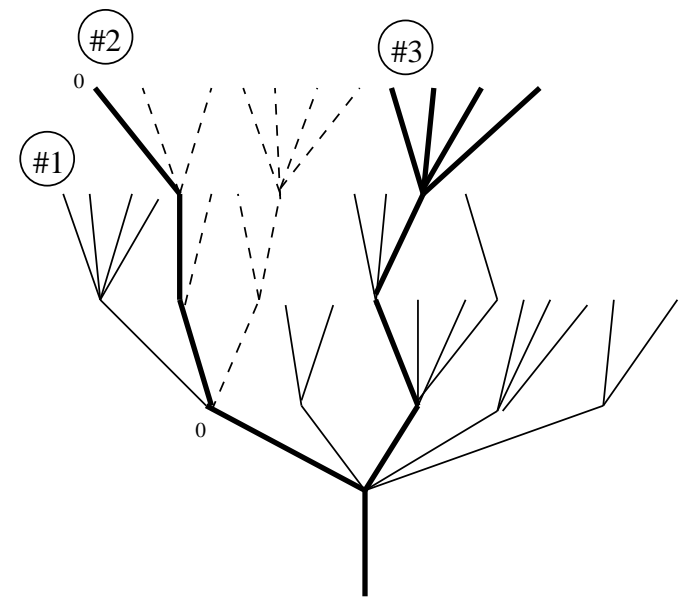

Figure 4: On the value of lazy evaluation: Every node corresponds to a partially constructed graph and its associated (lazy) term. The root node is just the initial domain. Every link leads from a graph to a graph which has been extended by one more vertex. In (\#1), no algebraic calculations are performed, as graph generation gets stuck with partial graphs that cannot be completed to final planar graphs. In (\#2), a planar graph could be generated, and term algebra forces all the promises for calculations down to the root to be fulfilled (bold lines). However, the result is zero, coming from a zero at level two that is discovered in this step only. All the other graphs and partial graphs that would also derive from this node are then not even generated, as the 'known zero value' information is used in graph generation. In (\#3), we get final nonzero contributions. Note furthermore that intermediary results down the path to the root are only calculated once! 


\section{A.5.4 Implementation}

Due to memory access being a major bottleneck in today's CPUs, and the hierarchical structure of caches in computer systems, one should take some care not to unnecessarily waste space with data representations. However, one can over-optimize here - tight data representations frequently come at the expense of more code. In the present implementation, we use a single 8-bit byte (char) to represent legs within the term algebra. The most significant bit encodes whether a leg is of type $i j k$ or $a b c$, the other bits encode the 'name' of the leg (a number). This works because fermionic $A B C$ legs can only occur at the ends of chains of gamma matrices, which are stored in special places. Leg type information is needed in two places: to determine which extra number factor to introduce when reducing $\delta_{m}^{m}$, and to determine whether a sign flip has to occur when the rule $\gamma^{a} \Pi^{ \pm}=\Pi^{\mp} \gamma^{a}, \gamma^{i} \Pi^{ \pm}=\Pi^{ \pm} \gamma^{i}$ is used. Every chain of gamma matrices in a term is encoded as a string (vector of bytes), where byte 0 and 1 denote the names of the fermionic ends; the high-bits of these two bytes encode whether the chain has an extra factor $\Pi^{ \pm}$at the left end as well as its particular type. All delta factors are likewise encoded in a string holding pairs of bytes. Epsilon factors $\epsilon_{i j k}$ combine three indices, hence we use one 30-bit signed integer per factor, while we use a string to hold nine indices for every $\epsilon_{m_{1} \ldots m_{9}}$ factor. As fermionic legs are just those appearing inside gamma chains, hence easy to discern from $\mathrm{SO}(3) i j k$ and $\mathrm{SO}(6) a b c$ legs, we only have to discern the latter two types. This we do by using the convention that all legs given a number in the range $0, \ldots 63$ (i.e. with non-set sixth bit) are of type $a$, while those in the range $64 \ldots 126$ are of type $i$.

On the workbench, we use extra-long arrays to hold these data and fill pointers remembering how many entries are momentarily valid. Furthermore, on the workbench, we have to hold additional information about 'hot' factors. A further subtlety concerns two special types of indices: first, we get $i j k$ indices not introduced by the legs of some vertex: we get these from the $\gamma^{i j k} \epsilon_{i j k}$ contribution to $\Pi^{ \pm}$when closing a gamma trace. Here, we take indices from the end of the numerical range (with 127 being treated as special, as it also denotes 'no index' in graph generation) $126,125, \ldots$, remembering how many such factors we already introduced for the present graph. Second, as we want to split the $a$ index to $Z, \bar{Z}, W, \bar{W}, Y, \bar{Y}$, we let indices in the range $58 \ldots 63$ denote these special indices. As these split index ranges are one-dimensional, they do not provide special handles associating them with parts of the term; we do not need them here anymore.

\section{A.6 On the Calculation}

The size of this calculation is larger by orders of magnitude than any other symbolic computation the authors are aware of. Such jumps in complexity are quite generically accompanied by the discovery of new effects that did not occur on smaller scales. In this particular case, removing flaws from the process of running such large calculations eventually turned out to be more demanding than removing bugs in the code. The main reasons were:

1. Without a dedicated supercomputer time budget for this project, computation time had to be borrowed from a variety of different sources. 
2. The job queuing systems used at some sites turned out to be well adapted to the needs of numerical simulations, but not symbolic computations.

3. Some especially hard parts of the calculation had to be split multiple times.

4. Perturbation theory calculations are notoriously hard to debug if the only sign of an error is that the overall sum of data from $O(10000)$ individual files, which take many CPU-years to produce, is nonsense.

All in all, performing the actual calculation took about half a year.

\section{A.6.1 Performance}

Profiling a three-loop sample calculation shows that with all of these tricks incorporated, the code spends most of the time in graph generation, but there is no single hot spot where further optimization may be useful. One might wonder whether it would be possible to further speed up graph generation by adding more heuristic checks that allow to determine early - given a set of remaining vertices that have to be added to a partially finished graph - whether there will be any way to eventually produce a graph with only one connected component carrying exactly one cell with open legs, the idea being that as soon as situations occur like legs of type $A$ and $B$ that will not be bound anywhere anymore going to different cells, one may stop graph generation early. Experiments have shown that this is an important optimization if the term algebra is so expensive that it slows down partial graph generation. As long as there is only very little dynamic memory management going on inside the graph engine, the extra effort to do such early checks is large enough to destroy any advantage of this approach. Again, this situation would change should reconfigurable logic become available on mainstream CPUs. Then, one might be able to perform such checks at very little extra cost.

In table 2 we display the sizes of some calculations, done in FORM and with gemstone - note that one should not try to naively deduce a statement about relative quality of these symbolic manipulation systems from such timings, as very different algorithms are used for the different systems. Despite the not unimpressive size of the number of non-zero contributions (roughly, the number of diagrams), one should not be misled by them: the hard part of the work lies in generating graphs, the vast majority of which turn out to give no contribution. This can be glimpsed from the fact that average gemstone term generation speed drops from about $640 / s$ for $Z Z$ at three loops to about $20 / s$ for the length-eleven chain at four loops, but can be as high as 1050/s at three loops or even 5100/s at four loops for individual sequences. If we assume that these 'fluctuations' in term generation speed are roughly in the ballpark of the square root of the number of total (mostly non-permissible) graphs per non-vanishing term, we may wildly guess that the total length-eleven chain calculation at four-loop order would have had somewhat like half a quadrillion possible Feynman graphs. At least, it is a funny coincidence to note that by doing the estimate this way from a 'measurement', one arrives at the same number $\left(4.3 \cdot 10^{14}\right)$ which is obtained by just multiplying the naive number of possible combinations for the four-times-four-legs sequence for the largest vertex class $\left(188^{4}\right)$ with the number of different vertex sequences 


\begin{tabular}{|llrrll|}
\hline $\begin{array}{l}\text { Initial } \\
\text { Chain }\end{array}$ & $\begin{array}{l}\text { Loop } \\
\text { Order }\end{array}$ & $\begin{array}{r}\text { Non-Zero } \\
\text { Terms }\end{array}$ & $\begin{array}{l}\text { Approx. Calc Time } \\
\text { (equiv. CPU-hours, } \\
\text { 2 GHz AMD Athlon) }\end{array}$ & System & $\begin{array}{l}\text { Determines } \\
\text { Dilatation } \\
\text { Operator }\end{array}$ \\
\hline \hline zz & 2 & 1150 & $1.3 \cdot 10^{-4}$ & gemstone & No \\
wwwwzzz & 2 & 4729 & $1.6 \cdot 10^{-4}$ & gemstone & Yes \\
N.A. & 2 & N.A. & 0.24 & gemstone & No \\
\hline \hline zz & 3 & 575916 & 1.6 & gemstone & Yes \\
wwzwzwz & 3 & 2764630 & 177 & FORM & Yes \\
N.A. & 3 & N.A. & 1051 & gemstone & No \\
\hline \hline zz & 4 & 736439974 & 4170 & gemstone & Wrapping 1 \\
wwzz & 4 & 1601839076 & 4161 & gemstone & Wrapping 2 \\
wzwz & 4 & 1669740684 & 11560 & gemstone & No \\
wwzwzz & 4 & 2777838350 & 88812 & gemstone & Upto Wrapping \\
wwwzzzzzwwz & 4 & 6638616118 & & & \\
\hline
\end{tabular}

Table 2: Sizes of various calculations

(338 834). As this second estimate does not take such factors as the initial chain length, multiple ways to add one vertex to a graph, and variations in complexity between vertex sequences into account, this is indeed only a coincidence.

\section{A.6.2 Distributed Computing}

Evidently, such four-loop calculations are far too demanding to be performed on a single machine. Fortunately, they naturally decompose into many individual independent parts one for each sequence of vertices - and hence are trivially parallelizable. (One may wonder how much time is wasted by doing graph generation over and over again for vertex sequences that share a long common beginning. With reasonable assumptions, one can estimate this spurious effort to contribute only at most a few per-cent to total calculation time.)

Profiling a three-loop calculation shows that while some vertex sequences can be calculated much faster than others, there is no clear jump in the distribution of calculation times of individual pieces. Nevertheless, about $50 \%$ of the work is done for $0.5 \%$ of all vertex sequences, while $90 \%$ of all sequences take only $10 \%$ of the time. This is shown in figure 5 .

It is tempting to try to provide additional mechanisms to further parallelize work done on individual chains, and the nature of the calculation with its many branchings suggests quite directly how one may achieve this. As we do not know a priori where the hot spots are, we want to be able to signal a running calculation to break itself up. So far, this is only supported very rudimentarily: if a running calculation process receives an alarm signal (SIGALRM, either sent externally or set as an alarm timeout via a command-line option), the signal handler sets a flag which tells the graph engine to record all work that has to be done from that instant on in a list of deferred calculations, which is then serialized, gzip- 


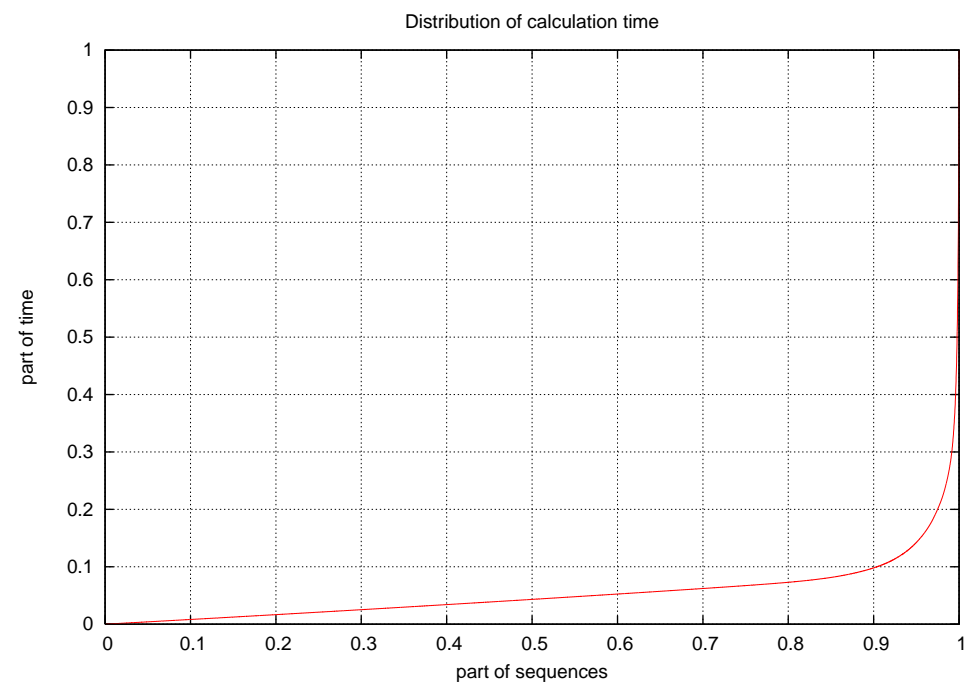

Figure 5: Accumulated calculation time vs. part of sequences for $Z Z$ at three-loop order

compressed, base64-encoded, and written to disk. ${ }^{13}$ (One may regard this as an ad-hoc approach to serializing a continuation.) The gemstone program is able to split a serialized calculation into multiple pieces of (hopefully) similar size.

As serialization of a running computation is a comparatively hairy action, we implemented as well a much simpler fallback method which involves splitting vertex classes into two parts. After identification of those pieces of the calculation that are hard to do, a complete expansion of all such $N$ vertex sequences of length $L$ into $N \cdot 2^{L}$ parts is indeed sufficient to bring the calculation time of every individual piece down to less than one week.

\section{A.6.3 Security}

So far, the gemstone system has only been used in controlled environments. Technically, it would be easy to extend the system in such a way that donated computer time from individual volunteers can be employed; the source already contains some first support for exchanging data via HTTP requests directly from the client. This raises some concerns in conjunction with both credibility of the overall result and security of participants' and organizers' computers. First of all, when even only one single machine produces an erroneous partial result - be it out of mischief or hardware unreliability, e.g. due to bad RAM or CPU overclocking, this will not be noticed until the final result is available. Hence, if such a scheme is feasible at all, then at least, every part of the calculation would have to be done twice by independent volunteers. Second, the problem of handling unexpectedly large pieces of the calculation has to be addressed. While it would be possible to let a client machine produce an image of the current state of its calculation and send it back so that it

\footnotetext{
${ }^{13}$ There are indications of problems with some versions of the Ocaml serializer, so this feature should be used with care.
} 
can be broken up and sent to further volunteers, this must not be permitted. Besides the occasionally prohibitive size of serialized calculations, a major concern is that the organizer of such a distributed calculation has little or no control over internal safety checks performed by the serializer. Thus, a mischievous participant may send in a specially manipulated data file which, upon re-serialization, may corrupt the Ocaml heap and in the worst case lead to arbitrary code execution for a different volunteer. The perhaps safest way to address this problem is to accept some redundant work and recursively decompose any large problem for which a client sends the information that it cannot be done within a given time-frame into smaller sub-problems by splitting vertex classes. This also has the big advantage that one may exert full control over the locations of these splits - hardly possible if they are mostly controlled by a timeout.

\section{A.6.4 On the Code}

While the main program is written in Objective Caml for portability, some of the Ocaml source files are generated by Perl and Lisp, especially if they had to be translated from Mathematica. (For some pieces for which speed is not essential, the machine generated code is quite ad-hoc and certainly not as efficient as it might be.) Thus, building the entire system will unfortunately require quite a large software development infrastructure.

As this system is work in progress, there are quite some places where the code is just as clean as it has to be in order to be maintainable; depending on further applications of this codebase, it may or may not become desirable to do some extensive re-structurings especially of the documentation in order to enhance readability.

\section{A.7 Five Loops}

Can this head-on approach be used to do the five loop calculation? One does not have to consider initial chains of length greater than 13 in order to fix the five-loop dilatation operator. As such a calculation may introduce at most nine extra indices from three $\epsilon^{i j k}$ via fermion loops of odd length, we would have to deal with at most $3 \cdot 10+9+13=42$ indices, well below the built-in maximum of 63 in gemstone. Furthermore, if one is interested only in the result and does not care about final states with fermions, which will cancel in the total sum anyway, small changes to gemstone that drop these terms would make it run with a very small memory footprint, hence maybe even suited for networked video game consoles.

While gemstone not only is considerable faster than any other method used so far, but also scales better, the relative computational effort for three loops is 3000 times larger than for two loops, and the effort for four loops is again larger by a factor 60000 . So it is perhaps not unreasonable to estimate that the size of the five-loop calculation should be at least one million times larger than the largest ones presented here. Hence, one would probably need something in the range of 100-500 million fast machines to do it. This is roughly in the ballpark of the total technical capability of mankind today. 


\section{A.8 Results}

For the sake of reproducibility of our methods and findings, we include raw results of all the large four-loop computer calculations, as well as for the calculations determining the dilatation operator at two and three loops.

$W W W W Z Z Z$ at two loops:

$\begin{array}{lr}W W W W Z Z Z & -44 \\ W W W Z W Z Z & 26 \\ W W W Z Z W Z & 26 \\ W W Z W W Z Z & -8\end{array}$

$W W Z W Z W Z$ at three loops:

$\begin{array}{lr}W W W W Z Z Z & 16 \\ W W W Z W Z Z & -612 \\ W W W Z Z W Z & -612 \\ W W Z W W Z Z & -1464 \\ W W Z W Z W Z & 2672\end{array}$

$Z Z$ at four loops:

$W Z W Z$ at four loops:

$$
\begin{array}{lr}
W W Z Z & 88224 \\
W Z W Z & -88224
\end{array}
$$

$W W Z Z$ at four loops:

$$
\begin{array}{lr}
W W Z Z & -44112 \\
W Z W Z & 44112
\end{array}
$$

$W W Z W Z Z$ at four loops:

$\begin{array}{lr}W Z W Z W Z & 22291 \\ W W W Z Z Z & 12803 \\ W W Z W Z Z & -73356 \\ W W Z Z W Z & 38262\end{array}$


$W W W Z Z Z Z Z W W Z$ at four loops:

$\begin{array}{lr}W W W W W Z Z Z Z Z Z & -3448 \\ W W W W Z W Z Z Z Z Z & -5640 \\ W W W W Z Z W Z Z Z Z & 52 \\ W W W W Z Z Z W Z Z Z & 52 \\ W W W W Z Z Z Z W Z Z & -1000 \\ W W W W Z Z Z Z Z W Z & 22399 \\ W W W Z W W Z Z Z Z Z & 22615 \\ W W W Z W Z W Z Z Z Z & 24 \\ W W W Z W Z Z Z Z W Z & -80 \\ W W W Z Z W W Z Z Z Z & 76 \\ W W W Z Z W Z Z W Z Z & -32 \\ W W W Z Z W Z Z Z W Z & 928 \\ W W W Z Z Z W W Z Z Z & -28 \\ W W W Z Z Z W Z W Z Z & 376 \\ W W W Z Z Z W Z Z W Z & -5012 \\ W W W Z Z Z Z W W Z Z & -2968 \\ W W W Z Z Z Z W Z W Z & 21819 \\ W W W Z Z Z Z Z W W Z & -63260 \\ W W Z W W Z W Z Z Z Z & 22195 \\ W W Z W W Z Z W Z Z Z & -5064 \\ W W Z W W Z Z Z W Z Z & 928 \\ W W Z W W Z Z Z Z W Z & -104 \\ W W Z W Z W W Z Z Z Z & -5168 \\ W W Z W Z W Z W Z Z Z & 432 \\ W W Z W Z W Z Z W Z Z & -32 \\ W W Z W Z Z W W Z Z Z & -28 \\ W W Z W Z Z Z W W Z Z & -32\end{array}$

This last result receives contributions from individual sequences such as e.g.

$\begin{array}{lr}W W W Z Z Z Z W Z W Z & 3747744 \\ W W W W Z Z Z Z Z W Z & 3747744 \\ W W W Z W W Z Z Z Z Z & 3747744 \\ W W Z W W Z W Z Z Z Z & 3747744 \\ W W W Z Z Z Z Z W W Z & -292115824\end{array}$

(from the sequence $\left.-\frac{7}{4} V_{1,+1} V_{1,-1} V_{1,+1} V_{2,+0} V_{1,+1} V_{1,-1} V_{1,-1}\right)$ or

$W W W Z Z Z Z Z W W Z \quad 2673 / 560$

(from the sequence $-\frac{1}{6720} V_{1,+3} V_{1,+1} V_{1,+3} V_{2,+1} V_{1,-3} V_{1,-3} V_{1,-2}$ ). Hence the fact that the result contains only small, allowed, integer contributions should be regarded as a highly nontrivial first indication of its correctness. 


\section{B Perturbation expansion of effective Hamiltonian}

The effective Hamiltonian acting on the $\mathfrak{s u}(2)$ subsector is given by a perturbative expansion

$$
T=\sum_{k=0}^{\infty} T_{2 k} \quad \text { with } T_{2 k} \propto \frac{1}{M^{3 k-1}},
$$

where $T_{2 k}$ is called the $k$-loop contribution. As building blocks we need an operator $P_{E}$ which projects onto the subspace of states with free energy $E$, i.e.

$$
P_{E}|F\rangle=\delta_{E F}|F\rangle \quad \text { for eigenstate }|F\rangle \text { of } H_{0} \text { with eigenvalue } F
$$

and a "propagator"

$$
\Delta_{E}=\sum_{F \neq E} \frac{P_{F}}{E-F}
$$

With these definitions, the terms in (B.1) read as follows:

$$
\begin{aligned}
& T_{0}=H_{0} \\
& T_{2}=\sum_{E} P_{E}\left[V_{1} \Delta_{E} V_{1}+V_{2}\right] P_{E} \\
& T_{4}=\sum_{E} P_{E}\left[V_{1} \Delta_{E} V_{1} \Delta_{E} V_{1} \Delta_{E} V_{1}\right. \\
& \left.-V_{1} \Delta_{E}^{2} V_{1} P_{E} T_{2}\right] P_{E} \\
& T_{6}=\sum_{E} P_{E}\left[V_{1} \Delta_{E} V_{1} \Delta_{E} V_{1} \Delta_{E} V_{1} \Delta_{E} V_{1} \Delta_{E} V_{1}\right. \\
& +V_{1} \Delta_{E} V_{1} \Delta_{E} V_{1} \Delta_{E} V_{1} \Delta_{E} V_{2}+V_{1} \Delta_{E} V_{1} \Delta_{E} V_{1} \Delta_{E} V_{2} \Delta_{E} V_{1} \\
& +V_{1} \Delta_{E} V_{1} \Delta_{E} V_{2} \Delta_{E} V_{1} \Delta_{E} V_{1}+V_{1} \Delta_{E} V_{2} \Delta_{E} V_{1} \Delta_{E} V_{1} \Delta_{E} V_{1} \\
& +V_{2} \Delta_{E} V_{1} \Delta_{E} V_{1} \Delta_{E} V_{1} \Delta_{E} V_{1} \\
& +V_{1} \Delta_{E} V_{1} \Delta_{E} V_{2} \Delta_{E} V_{2}+V_{1} \Delta_{E} V_{2} \Delta_{E} V_{1} \Delta_{E} V_{2}+V_{1} \Delta_{E} V_{2} \Delta_{E} V_{2} \Delta_{E} V_{1} \\
& +V_{2} \Delta_{E} V_{1} \Delta_{E} V_{1} \Delta_{E} V_{2}+V_{2} \Delta_{E} V_{1} \Delta_{E} V_{2} \Delta_{E} V_{1}+V_{2} \Delta_{E} V_{2} \Delta_{E} V_{1} \Delta_{E} V_{1} \\
& +V_{2} \Delta_{E} V_{2} \Delta_{E} V_{2} \\
& -\left(V_{1} \Delta_{E}^{2} V_{1} \Delta_{E} V_{1} \Delta_{E} V_{1}+V_{1} \Delta_{E} V_{1} \Delta_{E}^{2} V_{1} \Delta_{E} V_{1}+V_{1} \Delta_{E} V_{1} \Delta_{E} V_{1} \Delta_{E}^{2} V_{1}\right. \\
& +V_{1} \Delta_{E}^{2} V_{1} \Delta_{E} V_{2}+V_{1} \Delta_{E}^{2} V_{2} \Delta_{E} V_{1}+V_{2} \Delta_{E}^{2} V_{1} \Delta_{E} V_{1} \\
& +V_{1} \Delta_{E} V_{1} \Delta_{E}^{2} V_{2}+V_{1} \Delta_{E} V_{2} \Delta_{E}^{2} V_{1}+V_{2} \Delta_{E} V_{1} \Delta_{E}^{2} V_{1} \\
& \left.+V_{2} \Delta_{E}^{2} V_{2}\right) P_{E} T_{2} \\
& \left.+V_{1} \Delta_{E}^{3} V_{1} P_{E} T_{2} P_{E} T_{2}-V_{1} \Delta_{E}^{2} V_{1} P_{E} T_{4}\right] P_{E}
\end{aligned}
$$




$$
\begin{aligned}
& T_{8}=\sum_{E} P\left[V_{1} \Delta V_{1} \Delta V_{1} \Delta V_{1} \Delta V_{1} \Delta V_{1} \Delta V_{1} \Delta V_{1}\right. \\
& +V_{1} \Delta V_{1} \Delta V_{1} \Delta V_{1} \Delta V_{1} \Delta V_{1} \Delta V_{2}+V_{1} \Delta V_{1} \Delta V_{1} \Delta V_{1} \Delta V_{1} \Delta V_{2} \Delta V_{1} \\
& +V_{1} \Delta V_{1} \Delta V_{1} \Delta V_{1} \Delta V_{2} \Delta V_{1} \Delta V_{1}+V_{1} \Delta V_{1} \Delta V_{1} \Delta V_{2} \Delta V_{1} \Delta V_{1} \Delta V_{1} \\
& +V_{1} \Delta V_{1} \Delta V_{2} \Delta V_{1} \Delta V_{1} \Delta V_{1} \Delta V_{1}+V_{1} \Delta V_{2} \Delta V_{1} \Delta V_{1} \Delta V_{1} \Delta V_{1} \Delta V_{1} \\
& +V_{2} \Delta V_{1} \Delta V_{1} \Delta V_{1} \Delta V_{1} \Delta V_{1} \Delta V_{1} \\
& +V_{1} \Delta V_{1} \Delta V_{1} \Delta V_{1} \Delta V_{2} \Delta V_{2}+V_{1} \Delta V_{1} \Delta V_{1} \Delta V_{2} \Delta V_{1} \Delta V_{2}+V_{1} \Delta V_{1} \Delta V_{1} \Delta V_{2} \Delta V_{2} \Delta V_{1} \\
& +V_{1} \Delta V_{1} \Delta V_{2} \Delta V_{1} \Delta V_{1} \Delta V_{2}+V_{1} \Delta V_{1} \Delta V_{2} \Delta V_{1} \Delta V_{2} \Delta V_{1}+V_{1} \Delta V_{1} \Delta V_{2} \Delta V_{2} \Delta V_{1} \Delta V_{1} \\
& +V_{1} \Delta V_{2} \Delta V_{1} \Delta V_{1} \Delta V_{1} \Delta V_{2}+V_{1} \Delta V_{2} \Delta V_{1} \Delta V_{1} \Delta V_{2} \Delta V_{1}+V_{1} \Delta V_{2} \Delta V_{1} \Delta V_{2} \Delta V_{1} \Delta V_{1} \\
& +V_{1} \Delta V_{2} \Delta V_{2} \Delta V_{1} \Delta V_{1} \Delta V_{1}+V_{2} \Delta V_{1} \Delta V_{1} \Delta V_{1} \Delta V_{1} \Delta V_{2}+V_{2} \Delta V_{1} \Delta V_{1} \Delta V_{1} \Delta V_{2} \Delta V_{1} \\
& +V_{2} \Delta V_{1} \Delta V_{1} \Delta V_{2} \Delta V_{1} \Delta V_{1}+V_{2} \Delta V_{1} \Delta V_{2} \Delta V_{1} \Delta V_{1} \Delta V_{1}+V_{2} \Delta V_{2} \Delta V_{1} \Delta V_{1} \Delta V_{1} \Delta V_{1} \\
& +V_{1} \Delta V_{1} \Delta V_{2} \Delta V_{2} \Delta V_{2}+V_{1} \Delta V_{2} \Delta V_{1} \Delta V_{2} \Delta V_{2}+V_{1} \Delta V_{2} \Delta V_{2} \Delta V_{1} \Delta V_{2} \\
& +V_{1} \Delta V_{2} \Delta V_{2} \Delta V_{2} \Delta V_{1}+V_{2} \Delta V_{1} \Delta V_{1} \Delta V_{2} \Delta V_{2}+V_{2} \Delta V_{1} \Delta V_{2} \Delta V_{1} \Delta V_{2} \\
& +V_{2} \Delta V_{1} \Delta V_{2} \Delta V_{2} \Delta V_{1}+V_{2} \Delta V_{2} \Delta V_{1} \Delta V_{1} \Delta V_{2}+V_{2} \Delta V_{2} \Delta V_{1} \Delta V_{2} \Delta V_{1} \\
& +V_{2} \Delta V_{2} \Delta V_{2} \Delta V_{1} \Delta V_{1} \\
& +V_{2} \Delta V_{2} \Delta V_{2} \Delta V_{2} \\
& \text { - }\left(V_{1} \Delta V_{1} \Delta V_{1} \Delta V_{1} \Delta V_{1} \Delta^{2} V_{1}+V_{1} \Delta V_{1} \Delta V_{1} \Delta V_{1} \Delta^{2} V_{1} \Delta V_{1}+V_{1} \Delta V_{1} \Delta V_{1} \Delta^{2} V_{1} \Delta V_{1} \Delta V_{1}\right. \\
& +V_{1} \Delta V_{1} \Delta^{2} V_{1} \Delta V_{1} \Delta V_{1} \Delta V_{1}+V_{1} \Delta^{2} V_{1} \Delta V_{1} \Delta V_{1} \Delta V_{1} \Delta V_{1} \\
& +V_{1} \Delta V_{1} \Delta V_{1} \Delta V_{1} \Delta^{2} V_{2}+V_{1} \Delta V_{1} \Delta V_{1} \Delta V_{2} \Delta^{2} V_{1}+V_{1} \Delta V_{1} \Delta V_{1} \Delta^{2} V_{1} \Delta V_{2} \\
& +V_{1} \Delta V_{1} \Delta V_{1} \Delta^{2} V_{2} \Delta V_{1}+V_{1} \Delta V_{1} \Delta V_{2} \Delta V_{1} \Delta^{2} V_{1}+V_{1} \Delta V_{1} \Delta V_{2} \Delta^{2} V_{1} \Delta V_{1} \\
& +V_{1} \Delta V_{1} \Delta^{2} V_{1} \Delta V_{1} \Delta V_{2}+V_{1} \Delta V_{1} \Delta^{2} V_{1} \Delta V_{2} \Delta V_{1}+V_{1} \Delta V_{1} \Delta^{2} V_{2} \Delta V_{1} \Delta V_{1} \\
& +V_{1} \Delta V_{2} \Delta V_{1} \Delta V_{1} \Delta^{2} V_{1}+V_{1} \Delta V_{2} \Delta V_{1} \Delta^{2} V_{1} \Delta V_{1}+V_{1} \Delta V_{2} \Delta^{2} V_{1} \Delta V_{1} \Delta V_{1} \\
& +V_{1} \Delta^{2} V_{1} \Delta V_{1} \Delta V_{1} \Delta V_{2}+V_{1} \Delta^{2} V_{1} \Delta V_{1} \Delta V_{2} \Delta V_{1}+V_{1} \Delta^{2} V_{1} \Delta V_{2} \Delta V_{1} \Delta V_{1} \\
& +V_{1} \Delta^{2} V_{2} \Delta V_{1} \Delta V_{1} \Delta V_{1}+V_{2} \Delta V_{1} \Delta V_{1} \Delta V_{1} \Delta^{2} V_{1}+V_{2} \Delta V_{1} \Delta V_{1} \Delta^{2} V_{1} \Delta V_{1} \\
& +V_{2} \Delta V_{1} \Delta^{2} V_{1} \Delta V_{1} \Delta V_{1}+V_{2} \Delta^{2} V_{1} \Delta V_{1} \Delta V_{1} \Delta V_{1} \\
& +V_{1} \Delta V_{1} \Delta V_{2} \Delta^{2} V_{2}+V_{1} \Delta V_{1} \Delta^{2} V_{2} \Delta V_{2}+V_{1} \Delta V_{2} \Delta V_{1} \Delta^{2} V_{2}+V_{1} \Delta V_{2} \Delta V_{2} \Delta^{2} V_{1} \\
& +V_{1} \Delta V_{2} \Delta^{2} V_{1} \Delta V_{2}+V_{1} \Delta V_{2} \Delta^{2} V_{2} \Delta V_{1}+V_{1} \Delta^{2} V_{1} \Delta V_{2} \Delta V_{2}+V_{1} \Delta^{2} V_{2} \Delta V_{1} \Delta V_{2} \\
& +V_{1} \Delta^{2} V_{2} \Delta V_{2} \Delta V_{1}+V_{2} \Delta V_{1} \Delta V_{1} \Delta^{2} V_{2}+V_{2} \Delta V_{1} \Delta V_{2} \Delta^{2} V_{1}+V_{2} \Delta V_{1} \Delta^{2} V_{1} \Delta V_{2} \\
& +V_{2} \Delta V_{1} \Delta^{2} V_{2} \Delta V_{1}+V_{2} \Delta V_{2} \Delta V_{1} \Delta^{2} V_{1}+V_{2} \Delta V_{2} \Delta^{2} V_{1} \Delta V_{1}+V_{2} \Delta^{2} V_{1} \Delta V_{1} \Delta V_{2} \\
& \left.+V_{2} \Delta^{2} V_{1} \Delta V_{2} \Delta V_{1}+V_{2} \Delta^{2} V_{2} \Delta V_{1} \Delta V_{1}+V_{2} \Delta V_{2} \Delta^{2} V_{2}+V_{2} \Delta^{2} V_{2} \Delta V_{2}\right) P T_{2} \\
& +\left(V_{1} \Delta V_{1} \Delta V_{1} \Delta^{3} V_{1}+V_{1} \Delta V_{1} \Delta^{2} V_{1} \Delta^{2} V_{1}+V_{1} \Delta V_{1} \Delta^{3} V_{1} \Delta V_{1}+V_{1} \Delta^{2} V_{1} \Delta V_{1} \Delta^{2} V_{1}\right. \\
& +V_{1} \Delta^{2} V_{1} \Delta^{2} V_{1} \Delta V_{1}+V_{1} \Delta^{3} V_{1} \Delta V_{1} \Delta V_{1} \\
& +V_{1} \Delta V_{1} \Delta^{3} V_{2}+V_{1} \Delta V_{2} \Delta^{3} V_{1}+V_{1} \Delta^{2} V_{1} \Delta^{2} V_{2}+V_{1} \Delta^{2} V_{2} \Delta^{2} V_{1} \\
& +V_{1} \Delta^{3} V_{1} \Delta V_{2}+V_{1} \Delta^{3} V_{2} \Delta V_{1}+V_{2} \Delta V_{1} \Delta^{3} V_{1}+V_{2} \Delta^{2} V_{1} \Delta^{2} V_{1} \\
& \left.+V_{2} \Delta^{3} V_{1} \Delta V_{1}+V_{2} \Delta^{3} V_{2}\right) P T_{2} P T_{2} \\
& -\left(V_{1} \Delta V_{1} \Delta V_{1} \Delta^{2} V_{1}+V_{1} \Delta V_{1} \Delta^{2} V_{1} \Delta V_{1}+V_{1} \Delta^{2} V_{1} \Delta V_{1} \Delta V_{1}\right. \\
& +V_{1} \Delta V_{1} \Delta^{2} V_{2}+V_{1} \Delta V_{2} \Delta^{2} V_{1}+V_{1} \Delta^{2} V_{1} \Delta V_{2}+V_{1} \Delta^{2} V_{2} \Delta V_{1}+V_{2} \Delta V_{1} \Delta^{2} V_{1}+V_{2} \Delta^{2} V_{1} \Delta V_{1} \\
& \left.+V_{2} \Delta^{2} V_{2}\right) P T_{4} \\
& -V_{1} \Delta^{4} V_{1} P T_{2} P T_{2} P T_{2} \\
& +V_{1} \Delta^{3} V_{1} P\left(T_{4} P T_{2}+T_{2} P T_{4}\right) \\
& \left.-V_{1} \Delta^{2} V_{1} P T_{6}\right] P
\end{aligned}
$$


For the actual computation it is convenient to split up the vertices $V_{1}$ and $V_{2}$ into parts of definite energy shift

$$
\left[H_{0}, V_{i, \delta E}\right]=\delta E V_{i, \delta E} \quad i=1,2
$$

Since this implies

$$
\begin{aligned}
\Delta_{E} V_{i, \delta E} & =V_{i, \delta E} \Delta_{E-\delta E} \\
P_{E} V_{i, \delta E} & =V_{i, \delta E} P_{E-\delta E}
\end{aligned}
$$

we are able to remove all projectors and propagators from the formulas above by moving them to the right end and using $P_{E} P_{F}=\delta_{E F} P_{E}$.

The PWMT vertices (2.1) have components

$$
V_{1}=\sum_{\substack{k=-3 \\ k \neq 0}}^{3} V_{1, k M} \quad \text { and } \quad V_{2}=\sum_{k=-4}^{4} V_{2, k M}
$$

where pieces with positive and negative energy shift are related by $\left(V_{i, \delta E}\right)^{\dagger}=V_{i,-\delta E}$. We now list the vertices as they read after planar normal ordering, i.e. all planar self-contractions of the vertices are already taken into account:

$$
\begin{aligned}
V_{1, M}= & -\frac{3}{\sqrt{8 M}} \varepsilon_{i j k} \operatorname{tr} b_{i}^{\dagger} b_{j}^{\dagger} b_{k}+\frac{2 i}{\sqrt{M}}\left(\gamma_{a}\right)_{\alpha \beta} \operatorname{tr} \theta_{\alpha}^{\dagger} \theta_{\beta}^{\dagger} a_{a}+i \sqrt{\frac{2}{M}}\left(\gamma_{i}\right)_{\alpha \beta} \operatorname{tr} \theta_{\alpha}^{\dagger}\left[b_{i}^{\dagger}, \theta_{\beta}\right] \\
V_{1,2 M}= & -\frac{2 i}{\sqrt{M}}\left(\gamma_{a}\right)_{\alpha \beta} \operatorname{tr} \theta_{\alpha}^{\dagger} \theta_{\beta}^{\dagger} a_{a}^{\dagger} \\
V_{1,3 M}= & +\frac{1}{\sqrt{8 M}} \varepsilon_{i j k} \operatorname{tr} b_{i}^{\dagger} b_{j}^{\dagger} b_{k}^{\dagger} \\
V_{2,0}= & \frac{99 N^{2}}{4}+13 N \operatorname{tr} a_{a}^{\dagger} a_{a}+7 N \operatorname{tr} b_{i}^{\dagger} b_{i} \\
& -\frac{1}{2 M^{2}} \operatorname{tr}\left[b_{i}^{\dagger}, b_{j}\right]\left[b_{i}^{\dagger}, b_{j}\right]+\frac{1}{8 M^{2}} \operatorname{tr}\left[b_{i}^{\dagger}, b_{i}\right]\left[b_{j}^{\dagger}, b_{j}\right]-\frac{1}{4 M^{2}} \operatorname{tr}\left[b_{i}^{\dagger}, b_{j}^{\dagger}\right]\left[b_{i}, b_{j}\right] \\
& -\frac{2}{M^{2}} \operatorname{tr}\left[a_{a}^{\dagger}, a_{b}\right]\left[a_{a}^{\dagger}, a_{b}\right]+\frac{1}{2 M^{2}} \operatorname{tr}\left[a_{a}^{\dagger}, a_{a}\right]\left[a_{b}^{\dagger}, a_{b}\right]-\frac{1}{M^{2}} \operatorname{tr}\left[a_{a}^{\dagger}, a_{b}^{\dagger}\right]\left[a_{a}, a_{b}\right] \\
& -\frac{1}{2 M^{2}} \operatorname{tr}\left[a_{a}^{\dagger}, b_{i}^{\dagger}\right]\left[a_{a}, b_{i}\right]-\frac{1}{2 M^{2}} \operatorname{tr}\left[a_{a}^{\dagger}, b_{i}\right]\left[a_{a}, b_{i}^{\dagger}\right] \\
V_{2, M}= & -\frac{13 N}{2} \operatorname{tr} a_{a}^{\dagger} a_{a}^{\dagger}+\frac{1}{M^{2}} \operatorname{tr}\left[a_{a}^{\dagger}, a_{b}^{\dagger}\right]\left[a_{a}^{\dagger}, a_{b}\right] \\
& -\frac{1}{4 M^{2}} \operatorname{tr}\left[a_{a}, b_{i}^{\dagger}\right]\left[a_{a}, b_{i}^{\dagger}\right]+\frac{1}{2 M^{2}} \operatorname{tr}\left[a_{a}^{\dagger}, b_{i}^{\dagger}\right]\left[a_{a}^{\dagger}, b_{i}\right] \\
V_{2,2 M}= & -\frac{7 N}{2} \operatorname{tr} b_{i}^{\dagger} b_{i}^{\dagger}+\frac{1}{4 M^{2}} \operatorname{tr}\left[b_{i}^{\dagger}, b_{j}^{\dagger}\right]\left[b_{i}^{\dagger}, b_{j}\right] \\
& -\frac{1}{4 M^{2}} \operatorname{tr}\left[a_{a}^{\dagger}, a_{b}^{\dagger}\right]\left[a_{a}^{\dagger}, a_{b}^{\dagger}\right]+\frac{1}{2 M^{2}} \operatorname{tr}\left[a_{a}^{\dagger}, b_{i}^{\dagger}\right]\left[a_{a}, b_{i}^{\dagger}\right] \\
V_{2,3 M}= & -\frac{1}{4 M^{2}} \operatorname{tr}\left[a_{a}^{\dagger}, b_{i}^{\dagger}\right]\left[a_{a}^{\dagger}, b_{i}^{\dagger}\right] \\
\left.V_{2,4 M}\right] & -\frac{1}{4 M^{2}} \operatorname{tr}\left[b_{i}^{\dagger}, b_{j}^{\dagger}\right]\left[b_{i}^{\dagger}, b_{j}^{\dagger}\right] \\
&
\end{aligned}
$$


These are the building blocks for planar graphs (see Fig. 1), e.g.

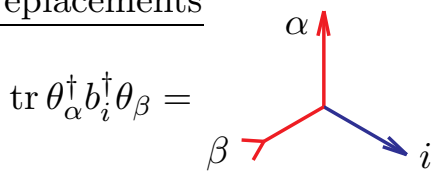

The arrows represent clockwise the oscillators in a trace, creation operators are out-going arrows and annihilation operators are in-going ones, the type of field determines the coloring. In the actual computer implementation we have split the $\mathfrak{s o}(6)$ index $a$ according to (2.6) into six different colors.

\section{Higher charges}

We now give the next two charges (3.15) beyond the spin-chain Hamiltonian $Q_{2}$ (4.1),(3.10). They are not uniquely determined from demanding that they commute with each other and with the Hamiltonian. The remaining freedom has been fixed in order to match the eigenvalue formulas (D.2).

$$
\begin{aligned}
Q_{3,2}= & i(\{0,1\}-\{1,0\}) \\
Q_{3,4}= & -8 i(\{0,1\}-\{1,0\})+2 i(\{0,1,2\}-\{2,1,0\}) \\
Q_{3,6}= & +3 i(\{0,1\}-\{1,0\})-28 i(\{0,1,2\}-\{2,1,0\}) \\
& -2 i(\{0,1,3\}+\{0,2,3\}-\{0,3,2\}-\{1,0,3\}) \\
& +5 i(\{0,1,2,3\}-\{3,2,1,0\})-i(\{0,2,1,3\}-\{1,0,3,2\}) \\
& -i(\{0,1,3,2\}-\{0,3,2,1\}+\{1,0,2,3\}-\{2,1,0,3\}) \\
Q_{3,8}= & -(751+\alpha) i(\{0,1\}-\{1,0\})+\frac{1}{2} i(701+4 \alpha)(\{0,1,2\}-\{2,1,0\}) \\
& +\frac{199}{4} i(\{0,1,3\}+\{0,2,3\}-\{0,3,2\}-\{1,0,3\}) \\
& -i(106+\alpha)(\{0,1,2,3\}-\{3,2,1,0\})+19 i(\{0,2,1,3\}-\{1,0,3,2\}) \\
& +\frac{1}{2} i(41-\alpha)(\{0,1,3,2\}-\{0,3,2,1\}+\{1,0,2,3\}-\{2,1,0,3\}) \\
& +2 i(\{0,1,4\}+\{0,3,4\}-\{0,4,3\}-\{1,0,4\})-4 i(\{0,1,3,4\}-\{1,0,4,3\}) \\
& -4 i(\{0,1,2,4\}+\{0,2,3,4\}-\{0,4,3,2\}-\{2,1,0,4\}) \\
& +14 i(\{0,1,2,3,4\}-\{4,3,2,1,0\})+i \alpha(\{0,3,2,1,4\}-\{1,0,2,4,3\}) \\
& +\frac{1}{4} i(1+2 \alpha)(\{0,2,1,3,2\}+\{1,0,2,1,3\}-\{1,0,3,2,1\}-\{2,1,0,3,2\}) \\
& -\frac{1}{2} i(6+\alpha)(\{0,1,3,2,4\}+\{0,2,1,3,4\}-\{1,0,4,3,2\}-\{2,1,0,4,3\}) \\
& -\frac{1}{2} i(6-\alpha)(\{0,1,2,4,3\}-\{0,4,3,2,1\}+\{1,0,2,3,4\}-\{3,2,1,0,4\})
\end{aligned}
$$




$$
\begin{aligned}
& Q_{4,2}=-\frac{4}{3}\{\}+\frac{8}{3}\{0\}-\frac{2}{3}(\{0,1\}+\{1,0\}) \\
& -\frac{2}{3}(\{0,2,1\}+\{1,0,2\})+\frac{2}{3}(\{0,1,2\}+\{2,1,0\}) \\
& Q_{4,4}=20\{\}-\frac{124}{3}\{0\}+\frac{34}{3}(\{0,1\}+\{1,0\})+\frac{8}{3}\{0,2\} \\
& -\frac{26}{3}(\{0,1,2\}+\{2,1,0\})+\frac{22}{3}(\{0,2,1\}+\{1,0,2\})-\frac{4}{3}\{1,0,2,1\} \\
& +2(\{0,1,2,3\}+\{3,2,1,0\})-\frac{2}{3}(\{0,2,1,3\}+\{1,0,3,2\}) \\
& -\frac{2}{3}(\{0,1,3,2\}+\{0,3,2,1\}+\{1,0,2,3\}+\{2,1,0,3\}) \\
& Q_{4,6}=-252\{\}+\frac{1604}{3}\{0\}-154(\{0,1\}+\{1,0\})-\frac{160}{3}\{0,2\} \\
& +\frac{332}{3}(\{0,1,2\}+\{2,1,0\})-\frac{244}{3}(\{0,2,1\}+\{1,0,2\})+\frac{64}{3}\{1,0,2,1\} \\
& -\frac{20}{3}\{0,3\}+4(\{0,1,3\}+\{0,2,3\}+\{0,3,2\}+\{1,0,3\}) \\
& -38(\{0,1,2,3\}+\{3,2,1,0\})+\frac{38}{3}(\{0,2,1,3\}+\{1,0,3,2\}) \\
& +\frac{34}{3}(\{0,1,3,2\}+\{0,3,2,1\}+\{1,0,2,3\}+\{2,1,0,3\}) \\
& +\frac{4}{3}(\{0,1,4,3\}+\{1,0,3,4\})-\frac{4}{3}(\{0,1,3,4\}+\{1,0,4,3\}) \\
& +\frac{4}{3}(\{0,2,1,4\}+\{0,2,4,3\}+\{0,3,2,4\}+\{1,0,2,4\}) \\
& -\frac{4}{3}(\{0,1,2,4\}+\{0,2,3,4\}+\{0,4,3,2\}+\{2,1,0,4\}) \\
& +6(\{0,1,2,3,4\}+\{4,3,2,1,0\})+\frac{2}{3}(\{0,3,2,1,4\}+\{1,0,2,4,3\}) \\
& +\frac{2}{3}(\{0,2,1,4,3\}+\{1,0,3,2,4\})+\frac{2}{3}(\{0,1,4,3,2\}+\{2,1,0,3,4\}) \\
& -\frac{4}{3}(\{0,2,1,3,2\}+\{1,0,2,1,3\}+\{1,0,3,2,1\}+\{2,1,0,3,2\}) \\
& -2(\{0,1,3,2,4\}+\{0,2,1,3,4\}+\{1,0,4,3,2\}+\{2,1,0,4,3\}) \\
& -2(\{0,1,2,4,3\}+\{0,4,3,2,1\}+\{1,0,2,3,4\}+\{3,2,1,0,4\}) \\
& Q_{4,8}=+\frac{2}{3}(4763+2 \alpha)\{\}-\frac{2}{3}(10349+16 \alpha)\{0\}+\frac{1}{3}(6139+22 \alpha)(\{0,1\}+\{1,0\}) \\
& +\frac{5}{3}(529+4 \alpha)\{0,2\}-\frac{835}{3}\{1,0,2,1\} \\
& -\frac{1}{3}(4391+12 \alpha)(\{0,1,2\}+\{2,1,0\})+(961-2 \alpha)(\{0,2,1\}+\{1,0,2\}) \\
& +\frac{2}{3}(271+2 \alpha)\{0,3\}-\frac{1}{3}(323+4 \alpha)(\{0,1,3\}+\{0,2,3\}+\{0,3,2\}+\{1,0,3\}) \\
& +\frac{1}{3}(1793+6 \alpha)(\{0,1,2,3\}+\{3,2,1,0\})-\frac{1}{6}(1163+16 \alpha)(\{0,2,1,3\}+\{1,0,3,2\}) \\
& +\frac{1}{2}(59+2 \alpha)(\{0,2,1,3,2\}+\{1,0,2,1,3\}+\{1,0,3,2,1\}+\{2,1,0,3,2\}) \\
& +12\{0,4\}-\frac{40}{3}\{0,2,4\}-8(\{0,1,4\}+\{0,3,4\}+\{0,4,3\}+\{1,0,4\}) \\
& -\frac{1}{6}(227+2 \alpha)(\{0,2,1,4\}+\{0,2,4,3\}+\{0,3,2,4\}+\{1,0,2,4\}) \\
& -\frac{1}{6}(955-4 \alpha)(\{0,1,3,2\}+\{0,3,2,1\}+\{1,0,2,3\}+\{2,1,0,3\}) \\
& +\frac{1}{6}(275+2 \alpha)(\{0,1,2,4\}+\{0,2,3,4\}+\{0,4,3,2\}+\{2,1,0,4\}) \\
& -\frac{1}{6}(207-4 \alpha)(\{0,1,4,3\}+\{1,0,3,4\})+\frac{1}{6}(271-4 \alpha)(\{0,1,3,4\}+\{1,0,4,3\})+
\end{aligned}
$$




$$
\begin{aligned}
& +\frac{1}{3}(145-\alpha)(\{0,1,2,4,3\}+\{0,4,3,2,1\}+\{1,0,2,3,4\}+\{3,2,1,0,4\}) \\
& -\frac{2}{3}(234+\alpha)(\{0,1,2,3,4\}+\{4,3,2,1,0\}) \\
& +\frac{8}{3}(\{0,3,2,4,3\}+\{1,0,2,1,4\})-\frac{1}{3}(41-2 \alpha)(\{0,2,1,4,3\}+\{1,0,3,2,4\}) \\
& -\frac{46}{3}(\{0,3,2,1,4\}+\{1,0,2,4,3\})-\frac{47}{3}(\{0,1,4,3,2\}+\{2,1,0,3,4\}) \\
& +\frac{1}{3}(148+\alpha)(\{0,1,3,2,4\}+\{0,2,1,3,4\}+\{1,0,4,3,2\}+\{2,1,0,4,3\}) \\
& -\frac{1}{6}(11-4 \alpha)(\{0,2,1,3,2,4\}+\{2,1,0,4,3,2\}) \\
& -\frac{19}{6}(\{0,1,3,2,4,3\}+\{1,0,2,1,3,4\}+\{1,0,4,3,2,1\}+\{3,2,1,0,4,3\}) \\
& +\frac{1}{2}(\{0,3,2,1,4,3\}+\{1,0,2,1,4,3\}+\{1,0,3,2,1,4\}+\{1,0,3,2,4,3\}) \\
& +\frac{4}{3}(\{0,1,4,5\}+\{1,0,5,4\})-\frac{4}{3}(\{0,1,5,4\}+\{1,0,4,5\}) \\
& -\frac{4}{3}(\{0,2,1,5\}+\{0,3,5,4\}+\{0,4,3,5\}+\{1,0,2,5\}) \\
& +\frac{4}{3}(\{0,1,2,5\}+\{0,3,4,5\}+\{0,5,4,3\}+\{2,1,0,5\}) \\
& +\frac{4}{3}(\{0,2,3,5,4\}+\{0,3,2,1,5\}+\{0,4,3,2,5\}+\{1,0,2,3,5\}) \\
& +\frac{4}{3}(\{0,2,1,3,5\}+\{0,2,4,3,5\}+\{0,3,2,5,4\}+\{1,0,3,2,5\}) \\
& +\frac{4}{3}(\{0,1,4,3,5\}+\{0,2,1,4,5\}+\{1,0,2,5,4\}+\{1,0,3,5,4\}) \\
& +\frac{4}{3}(\{0,1,3,5,4\}+\{0,2,1,5,4\}+\{1,0,2,4,5\}+\{1,0,4,3,5\}) \\
& +\frac{4}{3}(\{0,1,3,2,5\}+\{0,2,5,4,3\}+\{0,3,2,4,5\}+\{2,1,0,3,5\}) \\
& +\frac{4}{3}(\{0,1,2,5,4\}+\{0,1,5,4,3\}+\{1,0,3,4,5\}+\{2,1,0,4,5\}) \\
& -4(\{0,1,2,4,5\}+\{0,1,3,4,5\}+\{1,0,5,4,3\}+\{2,1,0,5,4\}) \\
& -4(\{0,1,2,3,5\}+\{0,2,3,4,5\}+\{0,5,4,3,2\}+\{3,2,1,0,5\}) \\
& +\frac{2}{3}(2-\alpha)(\{0,4,3,2,1,5\}+\{1,0,2,3,5,4\})+\frac{2}{3}(2+\alpha)(\{0,2,1,4,3,5\}+\{1,0,3,2,5,4\}) \\
& +\frac{4}{3}(\{0,2,1,3,5,4\}+\{0,3,2,1,5,4\}+\{1,0,2,4,3,5\}+\{1,0,4,3,2,5\}) \\
& +\frac{1}{6}(13+4 \alpha)(\{1,0,2,1,3,2\}+\{2,1,0,3,2,1\})-\frac{1}{6}(5+4 \alpha)(\{0,2,1,4,3,2\}+\{2,1,0,3,2,4\}) \\
& +\frac{1}{3}(2+\alpha)(\{0,1,4,3,2,5\}+\{0,3,2,1,4,5\}+\{1,0,2,5,4,3\}+\{2,1,0,3,5,4\}) \\
& +\frac{1}{3}(2-\alpha)(\{0,1,3,2,5,4\}+\{0,2,1,5,4,3\}+\{1,0,3,2,4,5\}+\{2,1,0,4,3,5\}) \\
& +\frac{56}{3}(\{0,1,2,3,4,5\}+\{5,4,3,2,1,0\})-\frac{16}{3}(\{0,1,3,2,4,5\}+\{2,1,0,5,4,3\}) \\
& +\frac{1}{3}(\{0,1,2,5,4,3\}+\{0,1,5,4,3,2\}+\{2,1,0,3,4,5\}+\{3,2,1,0,4,5\}) \\
& -\frac{1}{3}(18-\alpha)(\{0,1,2,3,5,4\}+\{0,5,4,3,2,1\}+\{1,0,2,3,4,5\}+\{4,3,2,1,0,5\}) \\
&
\end{aligned}
$$

\section{Spin chain spectrum}

In this appendix we list the eigenvalues $q_{2,3,4}$ of the charges $Q_{2,3,4}$ for spin-chains of length $L$ and magnon number $M$. The eigenvalues have been computed once by directly applying the corresponding operators to explicit states, and a second time by solving the Bethe 
equations for the momenta ${ }^{14}$ and using the following eigenvalue formulas

$$
q_{i}=\sum_{j=1}^{M} q_{i}\left(p_{j}\right)
$$

with

$$
\begin{aligned}
q_{2}(p)= & 8 \sin ^{2}\left(\frac{p}{2}\right)-32 \Lambda_{r} \sin ^{4}\left(\frac{p}{2}\right) \\
& +256 \Lambda_{r}^{2} \sin ^{6}\left(\frac{p}{2}\right)-2560 \Lambda_{r}^{3} \sin ^{8}\left(\frac{p}{2}\right) \\
q_{3}(p)= & 8 \sin (p) \sin ^{2}\left(\frac{p}{2}\right)-64 \Lambda_{r} \sin (p) \sin ^{4}\left(\frac{p}{2}\right) \\
& +640 \Lambda_{r}^{2} \sin (p) \sin ^{6}\left(\frac{p}{2}\right)-7168 \Lambda_{r}^{3} \sin (p) \sin ^{8}\left(\frac{p}{2}\right), \\
q_{4}(p)= & \frac{32}{3} \sin \left(\frac{3 p}{2}\right) \sin ^{3}\left(\frac{p}{2}\right)-128 \Lambda_{r} \sin \left(\frac{3 p}{2}\right) \sin ^{5}\left(\frac{p}{2}\right) \\
& +1536 \Lambda_{r}^{2} \sin \left(\frac{3 p}{2}\right) \sin ^{7}\left(\frac{p}{2}\right)-\frac{57344}{3} \Lambda_{r}^{3} \sin \left(\frac{3 p}{2}\right) \sin ^{9}\left(\frac{p}{2}\right) .
\end{aligned}
$$

These formulas are in accordance with the general all-loop expressions for $q_{i}$ given in 8 14, 25].

The stars "***" in the tables below indicate that the higher loop eigenvalues could not be determined due to wrapping issues. The value $q_{2,8}=-\frac{12771}{4} \Lambda_{r}^{3}$ for the state $(L, M)=(4,2)$ is also subject to wrapping, but was determined in a separate calculation (2.31). If we used the eigenvalue formula (D.2a) one would get the wrong value $q_{2,8}=-3054 \Lambda_{r}^{3}$.

"Singular" means that the Bethe equations become singular for the lowest order solution of the Bethe momenta $p_{i}$. In these cases one should use the inhomogeneous Bethe ansatz as in 8 .

Unpaired states are annihilated by $Q_{3}$, hence $q_{3}=0$. In these cases we give the parity of the corresponding multiplet in the column for $q_{3}$. If $Q_{3}$ does not annihilate a state, then it maps the state to the partner state of opposite parity. As the generic $Q_{3}$ and the parity operator do not commute (they anti-commute), we cannot diagonalize both operators simultaneously. In these cases we print the pair of eigenvalues of $Q_{3}$ which are always the negative of each other. All Bethe momenta are also opposite to each other for a degenerate pair of states.

\begin{tabular}{|c|c|l|l|l|l|}
\hline$L$ & $M$ & $q_{2}$ & $q_{3}$ & $q_{4}$ & Bethe momenta \\
\hline 4 & 2 & 12 & + & 0 & $p_{1}=\frac{2 \pi}{3}$ \\
& & $-48 \Lambda_{r}$ & $* * *$ & \\
& & $+336 \Lambda_{r}^{2}$ & & \\
& & $-\frac{12711}{4} \Lambda_{r}^{3}$ & & & \\
\hline 5 & 2 & 8 & & $\frac{16}{3}$ & \\
& & $-24 \Lambda_{r}$ & & $-32 \Lambda_{r}$ & $p_{1}=\frac{\pi}{2}$ \\
& & $+136 \Lambda_{r}^{2}$ & & \\
& & $-1024 \Lambda_{r}^{3}$ & & & \\
\hline
\end{tabular}

Table 3: Eigenvalues of $Q_{2}, Q_{3}, Q_{4}$

\footnotetext{
${ }^{14}$ We are grateful to V. Dippel for providing us some of these solutions from her unpublished work.
} 


\begin{tabular}{|c|c|c|c|c|c|}
\hline$L$ & $M$ & $q_{2}$ & $q_{3}$ & $q_{4}$ & Bethe momenta \\
\hline \multirow[t]{3}{*}{6} & \multirow[t]{2}{*}{2} & $\begin{array}{l}2(5-\sqrt{5}) \\
-(34-10 \sqrt{5}) \Lambda_{r} \\
+\frac{1}{5}(1170-414 \sqrt{5}) \Lambda_{r}^{2} \\
-\frac{1}{5}(10695-4134 \sqrt{5}) \Lambda_{r}^{3}\end{array}$ & + & $\begin{array}{l}-\frac{10}{3}(1-\sqrt{5}) \\
+12(5+3 \sqrt{5}) \Lambda_{r} \\
-6(127+65 \sqrt{5}) \Lambda_{r}^{2} \\
* * *\end{array}$ & $\begin{array}{l}p_{1}=\frac{2 \pi}{5} \\
p_{2}=-p_{1}\end{array}$ \\
\hline & & $\begin{array}{l}2(5+\sqrt{5}) \\
-(34+10 \sqrt{5}) \Lambda_{r} \\
+\frac{1}{5}(1170+414 \sqrt{5}) \Lambda_{r}^{2} \\
-\frac{1}{5}(10695+4134 \sqrt{5}) \Lambda_{r}^{3}\end{array}$ & + & $\begin{array}{l}-\frac{10}{3}(1+\sqrt{5}) \\
+12(5-3 \sqrt{5}) \Lambda_{r} \\
-6(127-65 \sqrt{5}) \Lambda_{r}^{2} \\
* * *\end{array}$ & $\begin{array}{l}p_{1}=\frac{4 \pi}{5} \\
p_{2}=-p_{1}\end{array}$ \\
\hline & 3 & $\begin{array}{l}12 \\
-36 \Lambda_{r} \\
+252 \Lambda_{r}^{2} \\
-2484 \Lambda_{r}^{3}\end{array}$ & - & $\begin{array}{l}-12 \\
+144 \Lambda_{r} \\
-1620 \Lambda_{r}^{2} \\
* * *\end{array}$ & "singular" \\
\hline \multirow[t]{3}{*}{7} & \multirow[t]{2}{*}{2} & $\begin{array}{l}4 \\
-6 \Lambda_{r} \\
+\frac{37}{2} \Lambda_{r}^{2} \\
-\frac{335}{4} \Lambda_{r}^{3} \\
\end{array}$ & - & $\begin{array}{l}\frac{8}{3} \\
-10 \Lambda_{r} \\
+\frac{81}{2} \Lambda_{r}^{2} \\
-\frac{759}{4} \Lambda_{r}^{3} \\
\end{array}$ & $\begin{array}{l}p_{1}=\frac{\pi}{3} \\
p_{2}=-p_{1}\end{array}$ \\
\hline & & $\begin{array}{l}12 \\
-42 \Lambda_{r} \\
+\frac{555}{2} \Lambda_{r}^{2} \\
-\frac{9465}{4} \Lambda_{r}^{3} \\
\end{array}$ & - & $\begin{array}{l}0 \\
-18 \Lambda_{r} \\
-\frac{513}{2} \Lambda_{r}^{2} \\
+\frac{13311}{4} \Lambda_{r}^{3}\end{array}$ & $\begin{array}{l}p_{1}=\frac{2 \pi}{3} \\
p_{2}=-p_{1}\end{array}$ \\
\hline & 3 & $\begin{array}{l}10 \\
-30 \Lambda_{r} \\
+200 \Lambda_{r}^{2} \\
-\frac{3565}{2} \Lambda_{r}^{3} \\
\end{array}$ & $\begin{array}{l}\mp \sqrt{15} \\
\pm 8 \sqrt{15} \Lambda_{r} \\
\mp 69 \sqrt{15} \Lambda_{r}^{2} \\
\pm 668 \sqrt{15} \Lambda_{r}^{3}\end{array}$ & $\begin{array}{l}-\frac{10}{3} \\
+50 \Lambda_{r} \\
-570 \Lambda_{r}^{2} \\
+\frac{13245}{2} \Lambda_{r}^{3}\end{array}$ & $\begin{array}{l}p_{1}= \pm 1.16 \pm 0.93 i \\
p_{2}= \pm 1.16 \mp 0.93 i \\
p_{3}=-p_{1}-p_{2}\end{array}$ \\
\hline \multirow[t]{8}{*}{8} & \multirow[t]{3}{*}{2} & $\begin{array}{l}3.01 \\
-3.32 \Lambda_{r} \\
+7.66 \Lambda_{r}^{2} \\
-27.30 \Lambda_{r}^{3}\end{array}$ & + & $\begin{array}{l}1.70 \\
-4.83 \Lambda_{r} \\
+14.81 \Lambda_{r}^{2} \\
-53.90 \Lambda_{r}^{3}\end{array}$ & $\begin{array}{l}p_{1}=\frac{2 \pi}{7} \\
p_{2}=-p_{1}\end{array}$ \\
\hline & & $\begin{array}{l}9.78 \\
-29.22 \Lambda_{r} \\
+167.64 \Lambda_{r}^{2} \\
-1256.15 \Lambda_{r}^{3}\end{array}$ & + & $\begin{array}{l}4.42 \\
-26.67 \Lambda_{r} \\
+176.12 \Lambda_{r}^{2} \\
-1204.33 \Lambda_{r}^{3}\end{array}$ & $\begin{array}{l}p_{1}=\frac{4 \pi}{7} \\
p_{2}=-p_{1}\end{array}$ \\
\hline & & $\begin{array}{l}15.21 \\
-59.45 \Lambda_{r} \\
+456.70 \Lambda_{r}^{2} \\
-4430.55 \Lambda_{r}^{3}\end{array}$ & + & $\begin{array}{l}-15.46 \\
+187.49 \Lambda_{r} \\
-2190.93 \Lambda_{r}^{2} \\
+26640.90 \Lambda_{r}^{3}\end{array}$ & $\begin{array}{l}p_{1}=\frac{6 \pi}{7} \\
p_{2}=-p_{1}\end{array}$ \\
\hline & \multirow[t]{2}{*}{3} & $\begin{array}{l}8 \\
-20 \Lambda_{r} \\
+112 \Lambda_{r}^{2} \\
-842 \Lambda_{r}^{3}\end{array}$ & $\begin{array}{l}\mp 4 \\
\pm 28 \Lambda_{r} \\
\mp 210 \Lambda_{r}^{2} \\
\pm 1743 \Lambda_{r}^{3}\end{array}$ & $\begin{array}{l}\frac{4}{3} \\
-4 \Lambda_{r} \\
+16 \Lambda_{r}^{2} \\
-\frac{52}{3} \Lambda_{r}^{3} \\
\end{array}$ & $\begin{array}{l}p_{1}= \pm 0.96 \pm 0.59 i \\
p_{2}= \pm 0.96 \mp 0.59 i \\
p_{3}=-p_{1}-p_{2}\end{array}$ \\
\hline & & $\begin{array}{l}12 \\
-36 \Lambda_{r} \\
+264 \Lambda_{r}^{2} \\
-2592 \Lambda_{r}^{3}\end{array}$ & - & $\begin{array}{l}-12 \\
+132 \Lambda_{r} \\
-1536 \Lambda_{r}^{2} \\
+19056 \Lambda_{r}^{3}\end{array}$ & "singular" \\
\hline & \multirow[t]{3}{*}{4} & $\begin{array}{l}6.49 \\
-7.56 \Lambda_{r} \\
+10.22 \Lambda_{r}^{2} \\
+10.25 \Lambda_{r}^{3} \\
\end{array}$ & + & $\begin{array}{l}-0.93 \\
+10.15 \Lambda_{r} \\
-55.32 \Lambda_{r}^{2} \\
+285.14 \Lambda_{r}^{3} \\
\end{array}$ & $\begin{array}{l}p_{1}=1.13+0.87 i \\
p_{2}=1.13-0.87 i \\
p_{3}=-p_{1} \\
p_{4}=-p_{2}\end{array}$ \\
\hline & & $\begin{array}{l}10.90 \\
-31.76 \Lambda_{r} \\
+249.76 \Lambda_{r}^{2} \\
-2538.27 \Lambda_{r}^{3} \\
\end{array}$ & + & $\begin{array}{l}-11.67 \\
+129.44 \Lambda_{r} \\
-1528.91 \Lambda_{r}^{2} \\
+19065.27 \Lambda_{r}^{3}\end{array}$ & $\begin{array}{l}p_{1}=2.98 \\
p_{2}=-1.07 i \\
p_{3}=-p_{1} \\
p_{4}=-p_{2}\end{array}$ \\
\hline & & $\begin{array}{l}22.60 \\
-88.68 \Lambda_{r} \\
+636.03 \Lambda_{r}^{2} \\
-5933.98 \Lambda_{r}^{3} \\
\end{array}$ & + & $\begin{array}{l}-8.73 \\
+148.40 \Lambda_{r} \\
-1951.77 \Lambda_{r}^{2} \\
+25244.26 \Lambda_{r}^{3}\end{array}$ & $\begin{array}{l}p_{1}=2.63 \\
p_{2}=1.52 \\
p_{3}=-p_{1} \\
p_{4}=-p_{2}\end{array}$ \\
\hline
\end{tabular}

Table 3: Eigenvalues of $Q_{2}, Q_{3}, Q_{4}$ (cont.) 


\begin{tabular}{|c|c|c|c|c|c|}
\hline$L$ & $\vec{M}$ & $q_{2}$ & $q_{3}$ & $q_{4}$ & Bethe momenta \\
\hline \multirow[t]{9}{*}{9} & \multirow[t]{3}{*}{2} & $\begin{array}{l}4(2-\sqrt{2}) \\
-(26-17 \sqrt{2}) \Lambda_{r} \\
+\frac{1}{8}(179-993 \sqrt{2}) \Lambda_{r}^{2} \\
-\frac{1}{32}(51376-36103 \sqrt{2}) \Lambda_{r}^{3}\end{array}$ & - & $\begin{array}{l}-\frac{8}{3}(1-\sqrt{2}) \\
+10(4-3 \sqrt{2}) \Lambda_{r} \\
-\frac{21}{4}(88-63 \sqrt{2}) \Lambda_{r}^{2} \\
+\frac{1}{48}(260000-184411 \sqrt{2}) \Lambda_{r}^{3}\end{array}$ & $\begin{array}{l}p_{1}=\frac{\pi}{4} \\
p_{2}=-p_{1}\end{array}$ \\
\hline & & $\begin{array}{l}8 \\
-20 \Lambda_{r} \\
+98 \Lambda_{r}^{2} \\
-639 \Lambda_{r}^{3}\end{array}$ & - & $\begin{array}{l}\frac{16}{3} \\
-32 \Lambda_{r} \\
+204 \Lambda_{r}^{2} \\
-\frac{4192}{3} \Lambda_{r}^{3}\end{array}$ & $\begin{array}{l}p_{1}=\frac{\pi}{2} \\
p_{2}=-p_{1}\end{array}$ \\
\hline & & $\begin{array}{l}4(2+\sqrt{2}) \\
-(26+17 \sqrt{2}) \Lambda_{r} \\
+\frac{1}{8}(179+993 \sqrt{2}) \Lambda_{r}^{2} \\
-\frac{1}{32}(51376+36103 \sqrt{2}) \Lambda_{r}^{3}\end{array}$ & - & $\begin{array}{l}-\frac{8}{3}(1+\sqrt{2}) \\
+10(4+3 \sqrt{2}) \Lambda_{r} \\
-\frac{21}{4}(88+63 \sqrt{2}) \Lambda_{r}^{2} \\
+\frac{1}{48}(260000-184411 \sqrt{2}) \Lambda_{r}^{3}\end{array}$ & $\begin{array}{l}p_{1}=\frac{3 \pi}{4} \\
p_{2}=-p_{1}\end{array}$ \\
\hline & \multirow[t]{3}{*}{3} & $\begin{array}{l}6.45 \\
-13.18 \Lambda_{r} \\
+60.73 \Lambda_{r}^{2} \\
-378.34 \Lambda_{r}^{3}\end{array}$ & $\begin{array}{l} \pm 3.44 \\
\mp 20.01 \Lambda_{r} \\
\pm 124.66 \Lambda_{r}^{2} \\
\mp 859.22 \Lambda_{r}^{3}\end{array}$ & $\begin{array}{l}2.67 \\
-15.38 \Lambda_{r} \\
+103.80 \Lambda_{r}^{2} \\
-748.03 \Lambda_{r}^{3}\end{array}$ & $\begin{array}{l}p_{1}=\mp 0.83 \mp 0.43 i \\
p_{2}=\mp 0.83 \pm 0.43 i \\
p_{3}=-p_{1}-p_{2}\end{array}$ \\
\hline & & $\begin{array}{l}11.04 \\
-33.50 \Lambda_{r} \\
+231.93 \Lambda_{r}^{2} \\
-2149.91 \Lambda_{r}^{3}\end{array}$ & $\begin{array}{l} \pm 2.99 \\
\mp 23.92 \Lambda_{r} \\
\pm 223.38 \Lambda_{r}^{2} \\
\mp 2345.23 \Lambda_{r}^{3}\end{array}$ & $\begin{array}{l}-6.89 \\
+83.02 \Lambda_{r} \\
-952.18 \Lambda_{r}^{2} \\
+11361.10 \Lambda_{r}^{3}\end{array}$ & $\begin{array}{l}p_{1}=\mp 1.28 \mp 1.26 i \\
p_{2}=\mp 1.28 \pm 1.26 i \\
p_{3}=-p_{1}-p_{2}\end{array}$ \\
\hline & & $\begin{array}{l}16.51 \\
-55.32 \Lambda_{r} \\
+383.33 \Lambda_{r}^{2} \\
-3494.75 \Lambda_{r}^{3}\end{array}$ & $\begin{array}{l} \pm 8.61 \\
\mp 53.35 \Lambda_{r} \\
\pm 414.82 \Lambda_{r}^{2} \\
\mp 3879.59 \Lambda_{r}^{3}\end{array}$ & $\begin{array}{l}-9.12 \\
+120.35 \Lambda_{r} \\
-1471.62 \Lambda_{r}^{2} \\
+18242.92 \Lambda_{r}^{3}\end{array}$ & $\begin{array}{l}p_{1}= \pm 2.98 \\
p_{2}= \pm 1.15 \\
p_{3}=-p_{1}-p_{2}\end{array}$ \\
\hline & \multirow[t]{3}{*}{4} & $\begin{array}{l}10 \\
-30 \Lambda_{r} \\
+220 \Lambda_{r}^{2} \\
-\frac{4185}{2} \Lambda_{r}^{3} \\
\end{array}$ & $\begin{array}{l}\mp \sqrt{11} \\
\pm \frac{84}{11} \sqrt{11} \Lambda_{r} \\
\mp \frac{8495}{121} \sqrt{11} \Lambda_{r}^{2} \\
\pm \frac{978121}{1331} \sqrt{11} \Lambda_{r}^{3}\end{array}$ & $\begin{array}{l}-\frac{22}{3} \\
+86 \Lambda_{r} \\
-982 \Lambda_{r}^{2} \\
+\frac{23371}{2} \Lambda_{r}^{3} \\
\end{array}$ & $\begin{array}{l}p_{1}= \pm 2.63 \\
p_{2}=\mp 0.53 \pm 0.88 i \\
p_{3}=\mp 1.77 \\
p_{4}=-p_{1}-p_{2}-p_{3}\end{array}$ \\
\hline & & $\begin{array}{l}4(3-\sqrt{3}) \\
-18(2-\sqrt{3}) \Lambda_{r} \\
+\frac{1}{2}(456-255 \sqrt{3}) \Lambda_{r}^{2} \\
-\frac{1}{4}(7908-4541 \sqrt{3}) \Lambda_{r}^{3}\end{array}$ & - & $\begin{array}{l}0 \\
+(24-12 \sqrt{3}) \Lambda_{r} \\
-(360-198 \sqrt{3}) \Lambda_{r}^{2} \\
+\frac{1}{2}(9540-5417 \sqrt{3}) \Lambda_{r}^{3}\end{array}$ & $\begin{array}{l}p_{1}=0.94+0.55 i \\
p_{2}=0.94-0.55 i \\
p_{3}=-p_{1} \\
p_{4}=-p_{2}\end{array}$ \\
\hline & & $\begin{array}{l}4(3+\sqrt{3}) \\
-18(2+\sqrt{3}) \Lambda_{r} \\
+\frac{1}{2}(456+255 \sqrt{3}) \Lambda_{r}^{2} \\
-\frac{1}{4}(7908+4541 \sqrt{3}) \Lambda_{r}^{3}\end{array}$ & - & $\begin{array}{l}0 \\
+(24-12 \sqrt{3}) \Lambda_{r} \\
-(360-198 \sqrt{3}) \Lambda_{r}^{2} \\
+\frac{1}{2}(9540+5417 \sqrt{3}) \Lambda_{r}^{3}\end{array}$ & $\begin{array}{l}p_{1}=2.28 \\
p_{2}=1.28 \\
p_{3}=-p_{1} \\
p_{4}=-p_{2}\end{array}$ \\
\hline
\end{tabular}

Table 3: Eigenvalues of $Q_{2}, Q_{3}, Q_{4}$ (cont.) 


\section{References}

[1] D. Berenstein, J. M. Maldacena and H. Nastase: Strings in flat space and pp waves from $N=4$ super Yang Mills, JHEP 0204 (2002) 013, hep-th/0202021.

[2] N. Kim, T. Klose and J. Plefka: Plane-wave matrix theory from $N=4$ super Yang-Mills on $R x S^{* * 3}$, Nucl. Phys. B 671 (2003) 359, hep-th/0306054.

[3] J. A. Minahan and K. Zarembo: The Bethe-ansatz for $N=4$ super Yang-Mills, JHEP 0303 (2003) 013, hep-th/0212208.

[4] N. Beisert, C. Kristjansen and M. Staudacher: The dilatation operator of $N=4$ super Yang-Mills theory, Nucl. Phys. B 664 (2003) 131, hep-th/0303060.

[5] N. Beisert: The complete one-loop dilatation operator of $N=4$ super Yang-Mills theory, Nucl. Phys. B 676 (2004) 3, hep-th/0307015 • N. Beisert and M. Staudacher: The N = 4 SYM integrable super spin-chain, Nucl. Phys. B 670 (2003) 439, hep-th/0307042.

[6] N. Beisert: The su(2|3) dynamic spin chain, Nucl. Phys. B 682 (2004) 487, hep-th/0310252.

[7] D. Serban and M. Staudacher: Planar $N=4$ gauge theory and the Inozemtsev long range spin chain, JHEP 0406 (2004) 001, hep-th/0401057.

[8] N. Beisert, V. Dippel and M. Staudacher: A novel long range spin chain and planar $N$ = 4 super Yang-Mills, JHEP 0407 (2004) 075, hep-th/0405001.

[9] N. Beisert: The dilatation operator of $N=4$ super Yang-Mills theory and integrability, Phys. Rept. 405 (2005) 1, hep-th/0407277.

[10] V. I. Inozemtsev: Integrable Heisenberg-van Vleck chains with variable range exchange, Phys. Part. Nucl. 34 (2003) 166, Fiz. Elem. Chast. Atom. Yadra 34 (2003) 332, hep-th/0201001.

[11] C. G. . Callan, H. K. Lee, T. McLoughlin, J. H. Schwarz, I. Swanson and X. Wu: Quantizing string theory in $A d S(5) x S^{* *} 5$ : Beyond the pp-wave, Nucl. Phys. B 673 (2003) 3, hep-th/0307032.

[12] S. Frolov and A. A. Tseytlin, Multi-spin string solutions in AdS(5) $x S^{* *} 5$, Nucl. Phys. B 668 (2003) 77, hep-th/0304255. • S. Frolov and A. A. Tseytlin: Rotating string solutions: AdS/CFT duality in non-supersymmetric sectors, Phys. Lett. B 570 (2003) 96, hep-th/0306143 • G. Arutyunov, S. Frolov, J. Russo and A. A. Tseytlin: Spinning strings in AdS(5) $x S^{* *} 5$ and integrable systems, Nucl. Phys. B 671 (2003) 3 , hep-th/0307191.

[13] D. J. Gross, A. Mikhailov and R. Roiban: Operators with large $R$ charge in $N=4$ Yang-Mills theory, Annals Phys. 301 (2002) 31, hep-th/0205066 • A. Santambrogio and D. Zanon: Exact anomalous dimensions of $N=4$ Yang-Mills operators with large $R$ charge, Phys. Lett. B 545 (2002) 425, hep-th/0206079. 
[14] G. Arutyunov, S. Frolov and M. Staudacher: Bethe ansatz for quantum strings, JHEP 0410 (2004) 016, hep-th/0406256.

[15] V. A. Kazakov, A. Marshakov, J. A. Minahan and K. Zarembo: Classical / quantum integrability in AdS/CFT, JHEP 0405 (2004) 024, hep-th/0402207.

[16] N. Beisert: Spin chain for quantum strings, hep-th/0409054.

[17] B. Eden, C. Jarczak and E. Sokatchev: A three-loop test of the dilatation operator in $N=4 S Y M$, hep-th/0409009.

[18] A. Vogt, S. Moch and J. A. M. Vermaseren: The three-loop splitting functions in QCD: The singlet case, Nucl. Phys. B 691 (2004) 129, hep-ph/0404111.

[19] A. V. Kotikov, L. N. Lipatov, A. I. Onishchenko and V. N. Velizhanin: Three-loop universal anomalous dimension of the Wilson operators in $N=4$ SUSY Yang-Mills model, Phys. Lett. B 595 (2004) 521, hep-th/0404092.

[20] K. Dasgupta, M. M. Sheikh-Jabbari and M. Van Raamsdonk: Matrix perturbation theory for M-theory on a PP-wave, JHEP 0205 (2002) 056, hep-th/0205185.

[21] T. Klose and J. Plefka: On the integrability of large $N$ plane-wave matrix theory, Nucl. Phys. B 679 (2004) 127, hep-th/0310232.

[22] M. Staudacher: The Factorized S-Matrix of CFT/AdS, hep-th/0412188.

[23] M. B. Halpern and C. B. Thorn: Large $N$ matrix mechanics on the light-cone, Int. J. Mod. Phys. A 17 (2002) 1517, hep-th/0111280.

[24] N. Kim and J. Plefka: On the spectrum of pp-wave matrix theory, Nucl. Phys. B 643 (2002) 31, hep-th/0207034 • K. Dasgupta, M. M. Sheikh-Jabbari and M. Van Raamsdonk: Protected multiplets of M-theory on a plane wave, JHEP 0209 (2002) 021, hep-th/0207050.

[25] G. Arutyunov and M. Staudacher: Matching higher conserved charges for strings and spins, JHEP 0403 (2004) 004, hep-th/0310182.

[26] J. A. M. Vermaseren: New features of FORM, math-ph/0010025

[27] See for example: Michael Dales, "The Proteus Processor - A Conventional CPU with Reconfigurable Functionality," 9th International Workshop on Field Programmable Logic and Applications, pp. 431 - 437, Springer-Verlag, September 1999 (also available as: http://www.cl.cam.ac.uk/users/mwd24/phd/bin/dales-fpl99.pdf).

[28] AT\&T's graph visualization package graphviz is available from http://www.graphviz.org. 
[29] For an overview over ocaml's performance in international contests, cf. http://caml.inria.fr/contests-eng.html

[30] X. Leroy, "Le système Caml Special Light: modules et compilation efficace en Caml," INRIA research report Nov 1995, 2721. URL: http://pauillac.inria.fr/ xleroy/publi/caml-special-light-rr.ps.gz; The ocaml website is http://www.ocaml.org.

[31] W. T. Sullivan, D. Werthimer, et al., "A new major SETI project based on Project Serendip data and 100,000 personal computers," Astronomical and Biochemical Origins and the Search for Life in the Universe, Proc. of the Fifth Intl. Conf. on Bioastronomy = IAU Colloq. No. 161, eds. C.B. Cosmovici, S. Bowyer, and D. Werthimer (Publisher: Editrice Compositori, Bologna, Italy). The project's web page URL is http://setiathome.ssl.berkeley.edu.

[32] The URL of the "Great Internet Mersenne Prime Search" project is http://www.mersenne.org/prime.htm 